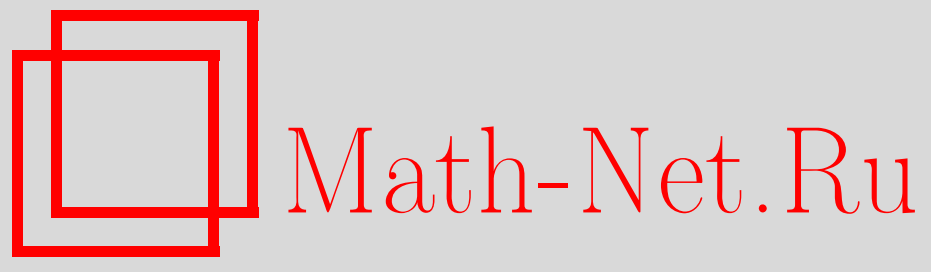

Н. М. Боголюбов, К. Л. Малышев, Интегрируемые модели и комбинаторика, УМH, 2015, том 70, выпуск 5, 3-74 DOI: https://doi.org/10.4213/rm9651

Использование Общероссийского математического портала Math-Net.Ru подразумевает, что вы прочитали и согласны с пользовательским соглашением http://www . mathnet.ru/rus/agreement

Параметры загрузки:

IP: 54.164 .48 .24

26 апреля 2023 г., 15:04:17

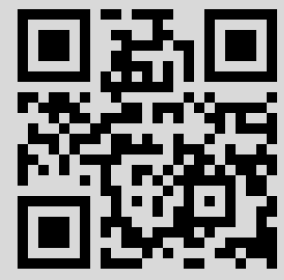




\title{
Интегрируемые модели и комбинаторика
}

\author{
Н. М. Боголюбов, К. Л. Малышев
}

Обсуждаются связи между квантовыми интегрируемыми моделями, решаемыми с помощью квантового метода обратной задачи, и некоторыми аспектами перечислительной комбинаторики и теории разбиений. В качестве основного примера рассматривается спиновая $X X Z$-цепочка Гейзенберга в предельных случаях нулевой и бесконечной анизотропии. Вычисляются форм-факторы и некоторые температурные корреляционные функции. Показано, что в специальной $q$-параметризации полученные форм-факторы представляют собой производящие функции плоских разбиений и решеточных самоизбегающих путей. Асимптотическое поведение корреляционных функций исследуется для случая большого числа узлов и умеренно большого числа спиновых возбуждений. При достаточно низкой температуре продемонстрирована связь корреляционных функций с теорией матричных интегралов.

Библиография: 125 названий.

Ключевые слова: корреляционные функции, магнетик Гейзенберга, четырехвершинная модель, плоские разбиения, производящие функции, симметрические функции.

DOI: $10.4213 / \mathrm{rm} 9651$

\section{СОДЕРЖАНИЕ}

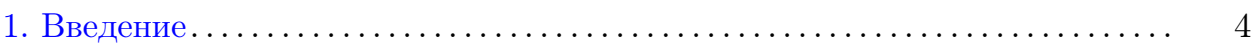

2. Разбиения, вершинные модели и их комбинаторные аспекты ........ 6

2.1. Разбиения, плоские разбиения и производящие функции плоских разбиений ....................................... 6

2.2. Четырехвершинная модель и квантовый метод обратной за-

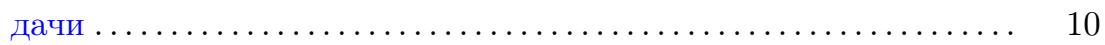

2.3. Статистическая сумма четырехвершинной модели и плоские

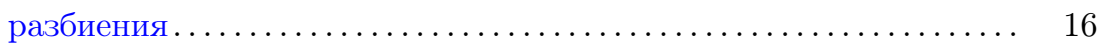

3. Гамильтониан $X X Z$-магнетика, векторы состояния и функции Шура . 21

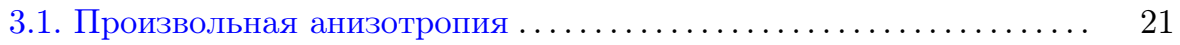

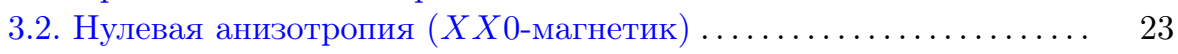

3.3. Бесконечная анизотропия (изинговский магнетик) .......... 24

3.4. О выборе эффективного гамильтониана при бесконечной анизо-

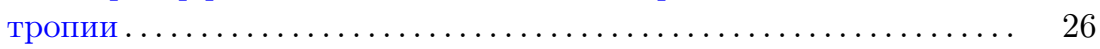

Исследование выполнено за счет гранта Российского научного фонда (проект № 14-11-00598). 
4. Корреляционные функции $X X Z$-магнетика при нулевой и бесконечной

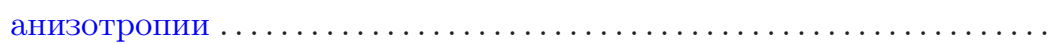

4.1. Форм-факторы и корреляционные функции при нулевой анизо-

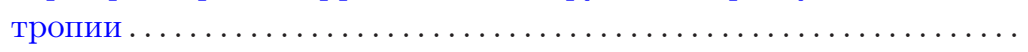

4.2. Форм-факторы и корреляционная функция выживания ферромагнитной струны при бесконечной анизотропии ............

5. q-биномиальные определители, решеточные пути и полустандартные

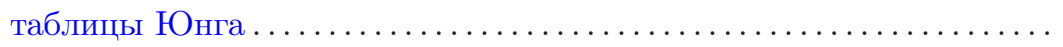

5.1. q-биномиальные определители и определитель куперберговского

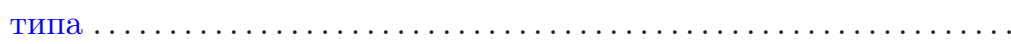

5.2. Производящая функция конфигурации “арбуз” и полустандарт-

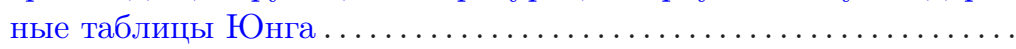

6. Форм-факторы и асимптотики корреляционных функций $X X Z$-магнетика при нулевой и бесконечной анизотропии ..............

6.1. Форм-факторы в $q$-параметризации и производящие функции

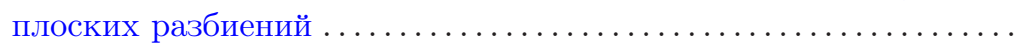

6.2. Асимптотики корреляционных функций при убывающей темпе-

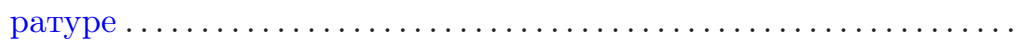

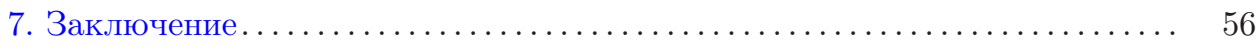

Приложение А. Вычисление определителя куперберговского типа....... 57

Приложение В. q-бозонная модель и комбинаторика ................ 61

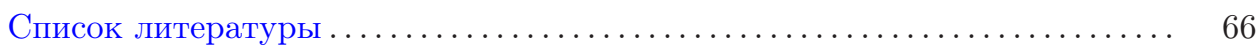

\section{1. Введение}

Квантовый метод обратной задачи (КМО3) [1]-[7] представляет эффективный подход к решению квантовых интегрируемых моделей низкоразмерной квантовой теории поля и статистической физики. Важной составной частью метода является алгебраический анзаи, Бете, который обобщает метод, сформулированный в оригинальном исследовании [8]. КМОЗ связан с такими бурно развивающимися областями современной математики и математической физики, как теория квантовых групп [9], маломерная топология [10], конформная [11]--[13] и суперсимметричная [14], [15] теории поля, а также теория представлений, связанная с уравнением Янга-Бакстера [16]-[18].

Существенное достоинство КМО3 состоит в возможности получать детальную информацию о поведении исследуемых моделей в конечном объеме и, как следствие, корректно выполнять переход к термодинамическому пределу. Перечислительная комбинаторика [19], [20] и ее актуальные задачи открывают новые возможности для приложений КМОЗ благодаря тому, что для корреляционных функций интегрируемых моделей в конечном объеме возникают связи с дискретной математикой. Особое положение в этом направлении исследований занимает точно решаемая шестивершинная модель на квадратной решетке [21]-[23] с граничными условиями типа доменной стенки [24]-[27]. В работе [28] было показано, что статистическая сумма модели, вычисленная 
ранее в [25], при определенной параметризации имеет отношение к перечислению знакопеременных матриц [29], [30] и к перечислению ромбовидных укладок [26], [31], [32]. Комбинаторика шестивершинной и относящихся к ней петлевых моделей подробно описана в работах [33], [34], а ее редукция, так называемая четырехвериинная модель, - в работе [35]. В последнее время активно исследуется проблема разделения фаз в модели с фиксированными граничными условиями [36]-[38], которая связана, в свою очередь, с теорией предельных распределений и вероятностных мер [39]-[42].

Теория плоских разбиений (трехмерных диаграмм Юнга) [20], [43] и теория случайных блужданий на решетках [44], [45], тесно связанные между собой [46]-[50], относятся к классическим проблемам комбинаторики [19], [20], [30], теории вероятностей [39], [40], [51] и теории симметрических функций [52]. Плоские разбиения естественно возникают при изучении математических структур, связанных с направленным протеканием [53], с ограненными кристаллами [54], [55], с плавлением [56], с топологическими струнами на многообразиях Калаби-Яо [57]. С точки зрения интегрируемых моделей плоские разбиения и случайные блуждания допускают как фермионное [54], [58]-[60], так и бозонное [61]-[63] описание.

Трансфер-матрица шестивершинной модели на торе и гамильтониан замкнутой цепочки спинов $1 / 2$ (широко известной как спиновая $X X Z$-цепочка Гейзенберга [64]) имеют общий набор собственных векторов [21]-[23]. Спиновая $X X Z$-цепочка Гейзенберга была решена как с помощью координатного анзаца Бете [65]-[67], так и с помощью квантового метода обратной задачи [1], [2]. Цепочка $X X Z$ характеризуется параметром анизотропии $\Delta$. Специальными случаями являются $\Delta=0$ ( $X X 0$-магнетик),$\Delta=1 / 2$ ("комбинаторная" точка [68]), $\Delta=1$ ( $X X X$-магнетик),$\Delta \rightarrow-\infty$ (изинговский предел [23]). Модель $X X 0$ связана с плоскими разбиениями [20], [30], с симметрическими функциями [52] и со случайными матрицами [69].

Вычисление корреляционных функций является актуальной задачей теории квантовых интегрируемых систем, и КМОЗ дает подход [70], [71] к получению точных ответов в конечном объеме и при различных граничных условиях. Вычисление корреляционных функций $X X Z$-модели с помощью алгебраического анзаца Бете потребовало серьезных усилий [24], [70]-[78].

Замечательным свойством $X X Z$-модели при $\Delta=0$ и при $\Delta \rightarrow-\infty$ является то, что ее многочастичные состояния допускают форму записи, содержащую симметрические функции Шура [52], а гамильтонианы можно рассматривать как генераторы случайных блужданий [58], [60]. Это дает возможность не только вычислять корреляционные функции в конечном объеме, но и давать комбинаторную интерпретацию полученным ответам. Например, форм-факторы некоторых операторов, вычисленные в так называемой $q$-параметризации, оказываются связанными с q-биномиальными определителями [79], что приводит к производящим функциям как плоских разбиений в ящике, так и самоизбегающих путей на решетке. Низкотемпературные асимптотики некоторых корреляторов в случае длинных, но конечных цепочек связаны [80] с матричными интегралами гауссовых ансамблей [81], [82].

Заметим, что корреляционные функции $X X 0$-магнетика интенсивно изучались в термодинамическом пределе [75], [83]-[86]. Мы же будем рассматривать асимптотическое поведение корреляционных функций при низкой температуре и для достаточно длинной цепочки, когда число спинов “вниз" (частиц) будет 
считаться большим, но умеренным. В этом пределе рассматриваемые корреляционные функции демонстрируют связь с матричными моделями [58], а также с двумерными $\mathscr{U}(N)$ калибровочными решеточными моделями [87], [88]. Результаты, полученные в [88], позволяют обсуждать для рассматриваемых нами спиновых систем возможность фазового перехода третьего рода, который известен для двумерной хромодинамики при $N \rightarrow \infty$ [87]. Возможность фазового перехода третьего рода обсуждалась и для непересекающихся броуновских движений на отрезке с периодическими, поглощающими и отражающими граничными условиями [89], а также для шестивершинной модели с граничным условием типа доменной стенки [90].

План работы следующий. Настоящий раздел 1 - вводный. Раздел 2 посвящен, в основном, четырехвершинной модели, а также связанным с ней статистическим суммам и скалярным произведениям векторов состояния. Вводятся плоские разбиения и их производящие функции, а также обсуждается интерпретация скалярных произведений в терминах плоских разбиений. В разделе 3 рассматриваются гамильтонианы и векторы состояния $X X Z$-магнетика Гейзенберга при $\Delta=0$ и при $\Delta \rightarrow-\infty$. Существенно, что используется возможность записи векторов состояния с помощью функций Шура. В разделе 4 обсуждается вычисление форм-факторов и специальных корреляционных функций с применением анзаца Бете и теории симметрических функций. А именно, в произвольной параметризации вычисляются температурные корреляционные функции типа выживания ферромагнитной струны и выживания доменной стенки. Кроме того, в разделе 4 обсуждаются случайные блуждания недружественных пешеходов. Для $X X 0$-модели Гейзенберга на периодической цепочке рассматривается корреляционная функция, являющаяся производящей функцией случайных блужданий недружественных пешеходов. Раздел 5 посвящен вычислению определителя специального вида. Выражение для этого определителя оказывается связанным с q-биномиальными и биномиальными определителями, что приводит, в свою очередь, к производящим функциям плоских разбиений и самоизбегающих решеточных путей. В разделе 6 устанавливается связь форм-факторов и асимптотик корреляторов с комбинаторикой плоских разбиений и перечислением самоизбегающих решеточных путей. При этом вычисления проводятся в $q$-параметризации и существенно опираются на соотношения, полученные в разделе 5. В разделе 7 дается заключение. Детали, опущенные при вычислении определителя в разделе 5 , приведены в приложении А. Ряд фактов, касающихся $q$-бозонной модели, ее корреляционных функций и плоских разбиений, представлен в приложении В. На всем протяжении предлагаемой работы считаются известными основные положения, связанные как с KMO3, так и с его применением к вычислению корреляционных функций интегрируемых моделей (см. [1]-[6], [70], [71]).

\section{2. Разбиения, вершинные модели и их комбинаторные аспекты}

\section{1. Разбиения, плоские разбиения и производящие функции плос-}

ких разбиений. Введем, следуя [52], ряд определений и соглашений. Жирными буквами будем обозначать наборы произвольных (комплексных) чисел следующего вида: например, $\mathbf{u}\left(\right.$ или $\mathbf{u}_{N}$ ) означает последовательность из $N$ чисел $\left(u_{1}, u_{2}, \ldots, u_{N}\right)$ и т. д. 
ОПРЕДЕЛЕНИЕ 1. Пусть $\overline{\mathbb{N}}$ - объединение множества натуральных чисел и нуля. Строгим разбиением $\boldsymbol{\mu}=\left(\mu_{1}, \mu_{2}, \ldots, \mu_{N}\right)$ назовем совокупность строго убывающих чисел $\mu_{i} \in \overline{\mathbb{N}}, 1 \leqslant i \leqslant N$, удовлетворяющих неравенству $P \geqslant$ $\mu_{1}>\mu_{2}>\cdots>\mu_{N} \geqslant 0$. Нестрогим разбиением $\boldsymbol{\lambda}=\left(\lambda_{1}, \lambda_{2}, \ldots, \lambda_{N}\right)$ назовем $N$-элементную последовательность нестрого убывающих чисел $\lambda_{i} \in \overline{\mathbb{N}}$, $1 \leqslant i \leqslant N$, удовлетворяющих неравенству $L \geqslant \lambda_{1} \geqslant \lambda_{2} \geqslant \cdots \geqslant \lambda_{N} \geqslant 0$.

Использование обозначений $\boldsymbol{\mu}$ и $\boldsymbol{\lambda}$, как независимо введенных, должно указывать на подразумеваемый тип разбиений. В приведенном определении фигурируют верхние границы $P, L \in \overline{\mathbb{N}}$. Хотя роль границ в определении не существенна, их уместно ввести, так как при обсуждении приложений они окажутся связанными с числами узлов конечных решеток, на которых будут рассматриваться физические модели.

Элемент разбиения, например $\lambda_{j}$, называется частъю разбиения. Длина $l(\boldsymbol{\lambda})$ разбиения равна количеству его частей. Сумма всех частей называется весом разбиения: $|\boldsymbol{\lambda}|=\sum_{i=1}^{N} \lambda_{i}$. Каждому строгому разбиению $\boldsymbol{\mu}$ можно поставить в соответствие нестрогое разбиение $\boldsymbol{\lambda}=\boldsymbol{\mu}-\boldsymbol{\delta}_{N}$, где $\boldsymbol{\delta}_{N}-$ строгое разбиение $(N-1, N-2, \ldots, 1,0)$. Иными словами, части разбиения $\boldsymbol{\lambda}$ определены равенством $\lambda_{j}=\mu_{j}-N+j$, где $1 \leqslant j \leqslant N$, и удовлетворяют неравенству $P+1-N \geqslant \lambda_{1} \geqslant \lambda_{2} \geqslant \cdots \geqslant \lambda_{N} \geqslant 0$ (т. е. $L=P+1-N$ в данном случае).

Каждому разбиению $\boldsymbol{\lambda}, l(\boldsymbol{\lambda})=N$, соответствует диаграмма Юнга, состоящая из $N$ столбцов таких, что число клеток в $i$-м столбце есть $\lambda_{i}$ для любого $i[19]$, [20]. Следуя [41], разбиению $\boldsymbol{\lambda}$ поставим в соответствие подмножество $\mathfrak{G}(\boldsymbol{\lambda})$ следующего вида:

$$
\mathfrak{G}(\boldsymbol{\lambda})=\left\{\lambda_{j}-j+\frac{1}{2} \mid 1 \leqslant j \leqslant N\right\} \subset \mathbb{Z}+\frac{1}{2} .
$$

Если прибавить $N-1 / 2$ к каждому элементу $\mathfrak{G}(\boldsymbol{\lambda})$, то получим части строгого разбиения $\boldsymbol{\mu}$, отвечающего $\boldsymbol{\lambda}$. Геометрическую интерпретацию элементов $\mathfrak{G}(\boldsymbol{\lambda})$ дает [41] диаграмма Юнга, расположенная под углом $\pi / 4$ к горизонтальной оси, как показано на рис. 1. Проведем вертикальные пунктирные линии, которые проектируют “верхушки” столбцов на горизонтальную ось. Координаты

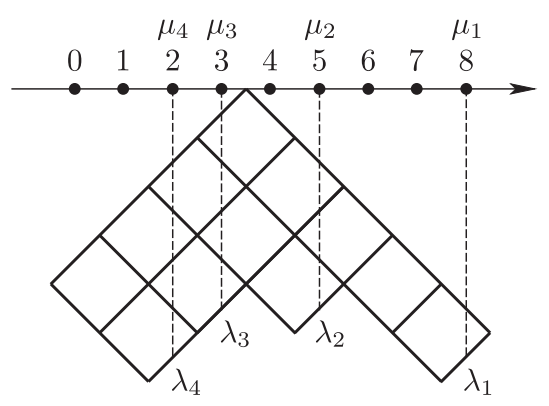

Рис. 1. Графический способ нахождения частей строгого разбиения $\boldsymbol{\mu}$ по диаграмме Юнга нестрогого разбиения $\boldsymbol{\lambda}$ 
точек пересечения пунктирных линий с горизонтальной осью дают части разбиения $\boldsymbol{\mu}$, которое соответствует $\mathfrak{G}(\boldsymbol{\lambda})$. Например, диаграмма на рис. 1 отвечает разбиениям $\boldsymbol{\lambda}=(5,3,2,2)$ и $\boldsymbol{\mu}=(8,5,3,2)$, где $\lambda_{i} \leqslant P-N+1=5$ при $P=8$ и $N=4$.

ОПРЕДЕЛЕНИЕ 2. Плоским разбиением формы $\boldsymbol{\lambda}$ назовем такое отображение $\boldsymbol{\pi}:(i, j) \mapsto \pi_{i j}$, где $i, j \geqslant 1$, из диаграммы Юнга (нестрогого) разбиения $\boldsymbol{\lambda}$ в множество $\overline{\mathbb{N}}$, что значения $\pi_{i j}$ не возрастают с увеличением как $i$, так и $j$.

Элементы $\pi_{i j}$ называются частями плоского разбиения, и $|\boldsymbol{\pi}|=\sum_{i, j} \pi_{i j}$ есть его объем (вес). Каждому плоскому разбиению соответствует трехмерная диаграмма Юнга, образуемая укладкой кубиков с единичной стороной, для которой высота столбца с координатами $(i, j)$ равна $\pi_{i j}$. Будем называть ящиком размера $L \times N \times P$ следующее подмножество целочисленной трехмерной решетки:

$$
\mathscr{B}(L, N, P)=\left\{(i, j, k) \in \overline{\mathbb{N}}^{3} \mid 0 \leqslant i \leqslant L, 0 \leqslant j \leqslant N, 0 \leqslant k \leqslant P\right\} .
$$

Принято говорить, что плоское разбиение $\boldsymbol{\pi}$ соответствует ящику $\mathscr{B}(L, N, P)$, если $i \leqslant L, j \leqslant N$ и $\pi_{i j} \leqslant P$ для всех кубиков диаграммы Юнга. Если части плоского разбиения $\boldsymbol{\pi}$ убывают вдоль каждого столбца и каждого ряда $\left(\pi_{i j}>\pi_{i+1, j}\right.$ и $\left.\pi_{i j}>\pi_{i, j+1}\right)$, то говорят, что $\pi$ есть строгое плоское разбиение, и пишут $\boldsymbol{\pi}_{\mathrm{spp}}$. Если части плоского разбиения $\boldsymbol{\pi}$ убывают вдоль каждого столбца $\left(\pi_{i j}>\pi_{i+1, j}\right)$, то говорят, что $\pi$ есть плоское разбиение, строгое по столбцам, и пишут $\boldsymbol{\pi}_{\text {сspp }}$. Плоское разбиение $\boldsymbol{\pi}$, соответствующее $\mathscr{B}(N, N, P)$, может быть преобразовано в строгое по столбцам плоское разбиение $\boldsymbol{\pi}_{\mathrm{csp}}$ в $\mathscr{B}(N, N, P+N-1)$ добавлением $(N \times N)$-матрицы

$$
\boldsymbol{\pi}_{\min }=\left(\begin{array}{cccc}
N-1 & N-1 & \ldots & N-1 \\
N-2 & N-2 & \ldots & N-2 \\
\vdots & \vdots & \ddots & \vdots \\
0 & 0 & \ldots & 0
\end{array}\right)
$$

Имеется связь между объемами разбиений $\boldsymbol{\pi}$ и $\boldsymbol{\pi}_{\mathrm{cspp}}:\left|\boldsymbol{\pi}_{\mathrm{cspp}}\right|=|\boldsymbol{\pi}|+\frac{N^{2}(N-1)}{2}$. Плоское разбиение $\boldsymbol{\pi}$, соответствующее $\mathscr{B}(N, N, P)$, может быть преобразовано в строгое плоское разбиение $\boldsymbol{\pi}_{\mathrm{spp}}$ в $\mathscr{B}(N, N, P+2 N-2)$ добавлением матрицы $\boldsymbol{\pi}_{\min }+\boldsymbol{\pi}_{\min }^{T}\left(\boldsymbol{\pi}_{\min }^{T}-\right.$ транспонированная матрица). Аналогичным образом связаны объемы разбиений: $\left|\boldsymbol{\pi}_{\mathrm{spp}}\right|=|\boldsymbol{\pi}|+N^{2}(N-1)$.

ОПРЕДЕЛЕНИЕ 3. Производящая функиия плоских разбиений в $\mathscr{B}(L, N, P)$ определяется как многочлен или формальный степенной ряд

$$
Z_{q}(L, N, P)=\sum_{\{\boldsymbol{\pi}\}} q^{|\boldsymbol{\pi}|},
$$

где $q^{|\boldsymbol{\pi}|}$ - статистический вес и суммирование идет по всем плоским разбиениям в $\mathscr{B}(L, N, P)$. Полный коэффициент при $q^{n}$ есть число плоских разбиений веса $n$. 
Производящая функция $Z_{q}(L, N, P)$ принимает следующий вид [52]:

$$
Z_{q}(L, N, P)=\prod_{j=1}^{L} \prod_{k=1}^{N} \prod_{i=1}^{P} \frac{1-q^{i+j+k-1}}{1-q^{i+j+k-2}}=\prod_{j=1}^{L} \prod_{k=1}^{N} \frac{1-q^{P+j+k-1}}{1-q^{j+k-1}} .
$$

В согласии с формулой Мак-Магона [43] имеется

$$
A(L, N, P)=\prod_{j=1}^{L} \prod_{k=1}^{N} \prod_{i=1}^{P} \frac{i+j+k-1}{i+j+k-2}=\prod_{j=1}^{L} \prod_{k=1}^{N} \frac{P+j+k-1}{j+k-1}
$$

плоских разбиений в ящике $\mathscr{B}(L, N, P)$. Правая часть (1) при $q \rightarrow 1$ стремится к $A(L, N, P)(2)$.

Производящая функция строгих по столбцам плоских разбиений в $\mathscr{B}(N, N, P)$ имеет вид

$$
Z_{q}^{\mathrm{cspp}}(N, N, P)=q^{N^{2}(N-1) / 2} \prod_{k=1}^{N} \prod_{j=1}^{N} \frac{1-q^{P+1+j-k}}{1-q^{j+k-1}}
$$

Предел $q \rightarrow 1$ дает число $A^{\operatorname{cspp}}(N, N, P)$ строгих по столбцам плоских разбиений в $\mathscr{B}(N, N, P)$ :

$$
A^{\operatorname{cspp}}(N, N, P)=\prod_{k=1}^{N} \prod_{j=1}^{N} \frac{P+1+j-k}{j+k-1}=\prod_{j=1}^{N} \frac{\Gamma(j) \Gamma(j+P+1)}{\Gamma(j+N) \Gamma(j+P+1-N)},
$$

где используется выражение для гамма-функции [91]. Заметим, что

$$
Z_{q}^{\mathrm{cspp}}(N, N, P)=q^{N^{2}(N-1) / 2} Z_{q}(N, N, P-N+1),
$$

и, следовательно, $A^{\operatorname{cspp}}(N, N, P)=A(N, N, P-N+1)$.

Производящая функция строгих плоских разбиений в $\mathscr{B}(N, N, P)$ имеет вид

$$
Z_{q}^{\mathrm{spp}}(N, N, P)=q^{N^{2}(N-1)} \prod_{k=1}^{N} \prod_{j=1}^{N} \frac{1-q^{P+3-j-k}}{1-q^{j+k-1}}
$$

и для нее выполняется соотношение

$$
Z_{q}^{\text {spp }}(N, N, P)=q^{N^{2}(N-1)} Z_{q}(N, N, P-2 N+2) .
$$

Число строгих плоских разбиений в $\mathscr{B}(N, N, P)$ возникает при $q \rightarrow 1$ :

$$
A^{\mathrm{spp}}(N, N, P)=\prod_{k=1}^{N} \prod_{j=1}^{N} \frac{P+3-j-k}{j+k-1},
$$

и получаем, что $A^{\mathrm{spp}}(N, N, P)=A(N, N, P-2 N+2)$. 
2.2. Четырехвершинная модель и квантовый метод обратной задачи. Шестивершинная модель на решетке описывает статистическую физику конфигураций стрелок на ребрах, соединяющих ближайшие узлы решетки. В каждом узле "сходятся" и "расходятся" по две стрелки. Каждая допустимая конфигурация стрелок на узле образует вершину, характеризующуюся статистическим весом $\omega_{p}, p=1,2, \ldots, 6$ (рис. 2). Заменяя ребра со стрелками, которые направлены "вверх" или "вправо", на толстые отрезки (а все остальные ребра на тонкие отрезки), можно получить альтернативное описание конфигураций вершин в терминах путей, соединяющих узлы решетки. Так как каждое ребро решетки может находиться только в двух состояниях (т. е. изображаться либо толстой, либо тонкой линией), то существует взаимно однозначное соответствие между допустимыми конфигурациями стрелок на решетке и наборами решеточных путей.
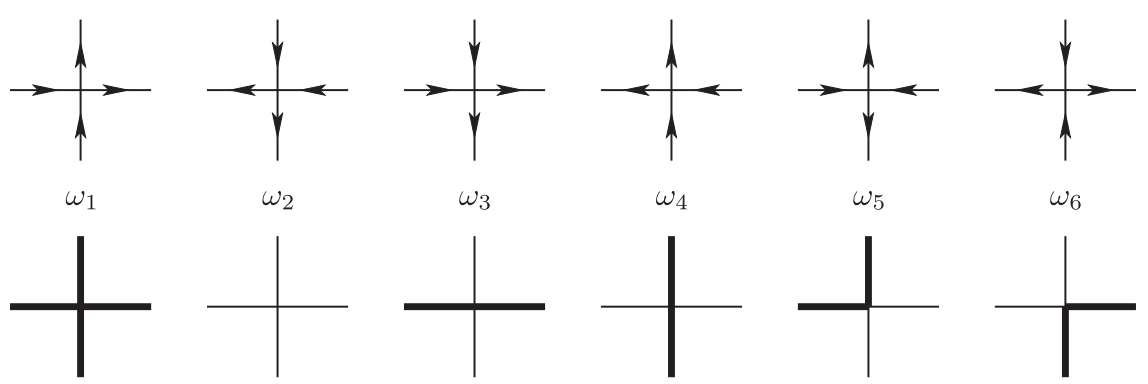

Рис. 2. Конфигурации стрелок (допустимые вершины) шестивершинной модели

В случае исключения двух вершин, $\omega_{1}=\omega_{3}=0$, шестивершинная модель сводится к четырехвершинной. Четырехвершинная модель рассматривалась в работах [92], [93], в которых была выявлена ее связь для периодического граничного условия со случайными укладками на полубесконечном цилиндре. Укладки возникают в некоторых моделях классической статистической физики, связанных с описанием паркетов, т. е. замощений поверхностей многоугольниками без зазоров и наложений. Мы будем интересоваться четырехвершинной моделью, в которой статистические веса третьей и четвертой вершины совпадают: $\omega_{a}=\omega_{2}, \omega_{b}=\omega_{4}, \omega_{c}=\omega_{5}=\omega_{6}$ (рис. 3 ).

Шестивершинная модель - одна из первых моделей, к которым были применены квантовый метод обратной задачи и алгебраический анзац Бете [1]. Модель задается на двумерной решетке, состоящей из $L \times(M+1)$ узлов: индексы $i, j, k$, изменяющиеся от 1 до $L$, будем использовать для нумерации узлов в горизонтальном направлении, индексы $l, m, n$, изменяющиеся от 0 до $M$, используем для нумерации узлов по вертикали. Каждому столбцу и каждой строке узлов поставим в соответствие локальное пространство, изоморфное $\mathbb{C}^{2}$. Вспомогательным пространством $\mathfrak{V}$ назовем пространство, отвечающее тензорному произведению всех локальных пространств, ассоциированных со столбцами узлов: $\mathfrak{V}=\left(\mathbb{C}^{2}\right)^{\otimes L}$, а квантовым - пространство $\mathfrak{H}$, отвечающее тензорному произведению всех локальных пространств, ассоциированных со 

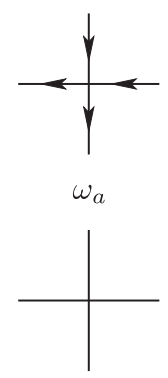

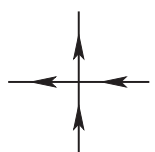

$\omega_{b}$

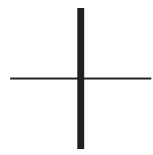

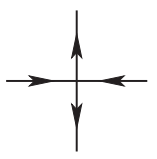

$\omega_{c}$

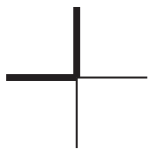

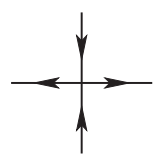

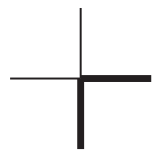

Рис. 3. Допустимые вершины четырехвершинной модели

строками узлов: $\mathfrak{H}=\left(\mathbb{C}^{2}\right)^{\otimes(M+1)}$ (терминология введена в [1]). Векторы $\left(\begin{array}{l}1 \\ 0\end{array}\right)$ (спин "вверх") и $\left(\begin{array}{l}0 \\ 1\end{array}\right)$ (спин "вниз") образуют естественный базис в пространстве $\mathbb{C}^{2}$. Для состояний на $l$-й строке введем специальные обозначения в виде стрелок, направленных влево или вправо: $|\leftarrow\rangle_{l} \equiv\left(\begin{array}{l}1 \\ 0\end{array}\right)_{l}$ и $|\rightarrow\rangle_{l} \equiv\left(\begin{array}{l}0 \\ 1\end{array}\right)_{l}$. Состояния, отвечающие столбцу с индексом $i$, обозначим стрелками, направленными вверх или вниз: $|\uparrow\rangle_{i} \equiv\left(\begin{array}{l}1 \\ 0\end{array}\right)_{i}$ и $|\downarrow\rangle_{i} \equiv\left(\begin{array}{l}0 \\ 1\end{array}\right)_{i}$.

Нам понадобится так называемый $L$-оператор, который действует в пространстве $\mathfrak{V} \otimes \mathfrak{H}$. Действие $L$-оператора нетривиально на одном-единственном узле, расположенном на пересечении $j$-го столбца и $n$-й строки. На остальных узлах $L$-оператор действует как единичный оператор. Введем локальные спиновые операторы $\sigma_{n}^{ \pm}=\left(\sigma_{n}^{x} \pm i \sigma_{n}^{y}\right) / 2$ и $\sigma_{n}^{z}$, зависящие от решеточного аргумента $n \in \mathbf{M}, \mathbf{M} \equiv\{0 \leqslant l \leqslant M \mid l \in \overline{\mathbb{N}}\}$. Операторы определены как $(M+1)$-кратные тензорные произведения и действуют нетривиально в $n$-м квантовом пространстве:

$$
\sigma_{n}^{\#}=\mathbb{I} \otimes \cdots \otimes \mathbb{I} \otimes \underbrace{\sigma^{\#}}_{n} \otimes \mathbb{I} \otimes \cdots \otimes \mathbb{I}
$$

где II обозначает единичную матрицу размера $2 \times 2$, а $\sigma^{\#}$ на $n$-м месте есть матрица Паули, $\sigma^{\#} \in \mathfrak{s u}(2)$ (индекс \# означает либо $x, y, z$, либо \pm ):

$$
\sigma^{x}=\left(\begin{array}{ll}
0 & 1 \\
1 & 0
\end{array}\right), \quad \sigma^{y}=\left(\begin{array}{cc}
0 & -i \\
i & 0
\end{array}\right), \quad \sigma^{z}=\left(\begin{array}{cc}
1 & 0 \\
0 & -1
\end{array}\right) .
$$

Перестановочные соотношения для операторов (9) имеют вид

$$
\left[\sigma_{n}^{+}, \sigma_{l}^{-}\right]=\delta_{n l} \sigma_{l}^{z}, \quad\left[\sigma_{n}^{z}, \sigma_{l}^{ \pm}\right]= \pm 2 \delta_{n l} \sigma_{l}^{ \pm},
$$

где $\delta_{n l}$ - символ Кронекера. Операторы (9) действуют над пространством состояний $\mathfrak{H}_{M+1} \equiv\left(\mathbb{C}^{2}\right)^{\otimes(M+1)}$. С учетом принятых определений рассмотрим $L$-оператор шестивершинной модели $L_{6 \mathrm{v}}(n \mid u)$, который не зависит от индекса 
в $\mathfrak{V}($ см. [1]):

$$
\begin{aligned}
L_{6 \mathrm{v}}(n \mid u)= & \check{q} \otimes\left(-u e^{\gamma \sigma_{n}^{z}}+u^{-1} e^{-\gamma \sigma_{n}^{z}}\right)+\hat{q} \otimes\left(-u e^{-\gamma \sigma_{n}^{z}}+u^{-1} e^{\gamma \sigma_{n}^{z}}\right) \\
& +2 \sinh (2 \gamma)\left(\sigma^{-} \otimes \sigma_{n}^{+}+\sigma^{+} \otimes \sigma_{n}^{-}\right) \\
= & \left(\begin{array}{cc}
-u e^{\gamma \sigma_{n}^{z}}+u^{-1} e^{-\gamma \sigma_{n}^{z}} & 2 \sinh (2 \gamma) \sigma_{n}^{-} \\
2 \sinh (2 \gamma) \sigma_{n}^{+} & -u e^{-\gamma \sigma_{n}^{z}}+u^{-1} e^{\gamma \sigma_{n}^{z}}
\end{array}\right)
\end{aligned}
$$

где $u \in \mathbb{C}$ и $\gamma \equiv(1 / 2) \operatorname{Arch} \Delta$ - параметры. $\mathrm{B}(10)$ введены проекторы на состояния со спинами "вниз" и "вверх":

$$
\hat{q} \equiv \frac{1}{2}\left(\mathbb{I}-\sigma^{z}\right), \quad \check{q} \equiv \frac{1}{2}\left(\mathbb{I}+\sigma^{z}\right), \quad \check{q}+\hat{q}=\mathbb{I} .
$$

Оператор $L_{6 \mathrm{v}}(n \mid u)(10)$ удовлетворяет сплетающему соотношению:

$$
\widetilde{R}(u, v)\left(L_{6 \mathrm{v}}(n \mid u) \otimes L_{6 \mathrm{v}}(n \mid v)\right)=\left(L_{6 \mathrm{v}}(n \mid v) \otimes L_{6 \mathrm{v}}(n \mid u)\right) \widetilde{R}(u, v) .
$$

Здесь $\widetilde{R}(u, v)$ обозначает матрицу размера $4 \times 4$ :

$$
\widetilde{R}(u, v)=\left(\begin{array}{cccc}
\widetilde{f}(v, u) & 0 & 0 & 0 \\
0 & \widetilde{g}(v, u) & 1 & 0 \\
0 & 1 & \widetilde{g}(v, u) & 0 \\
0 & 0 & 0 & \widetilde{f}(v, u)
\end{array}\right)
$$

где

$$
\widetilde{f}(v, u)=\frac{u^{2} e^{2 \gamma}-v^{2} e^{-2 \gamma}}{u^{2}-v^{2}}, \quad \widetilde{g}(v, u)=\frac{u v}{u^{2}-v^{2}}\left(e^{2 \gamma}-e^{-2 \gamma}\right)
$$

и $u, v \in \mathbb{C}$.

Подействуем на $L$-оператор (10) следующим преобразованием:

$$
\begin{aligned}
\check{L}(n \mid u) & =e^{h \sigma_{n}^{z}} e^{(\omega / 2) \sigma^{z}} L_{6 \mathrm{v}}(n \mid u) e^{-(\omega / 2) \sigma^{z}} \\
& =\left(\begin{array}{cc}
-u e^{(h+\gamma) \sigma_{n}^{z}}+u^{-1} e^{(h-\gamma) \sigma_{n}^{z}} & 2 \sinh (2 \gamma) e^{\omega+h \sigma_{n}^{z}} \sigma_{n}^{-} \\
2 \sinh (2 \gamma) e^{-\omega+h \sigma_{n}^{z}} \sigma_{n}^{+} & -u e^{(h-\gamma) \sigma_{n}^{z}}+u^{-1} e^{(h+\gamma) \sigma_{n}^{z}}
\end{array}\right) .
\end{aligned}
$$

Сплетающее соотношение для оператора $\check{L}(n \mid u)(15)$ соблюдается с преобразованной $R$-матрицей $\widetilde{R}(u, v)(13)$ :

$$
\begin{aligned}
\check{R}(u, v) & =\left(\mathbb{I} \otimes e^{-h \sigma^{z}}\right) \widetilde{R}(u, v)\left(\mathbb{I} \otimes e^{h \sigma^{z}}\right) \\
& =\left(\begin{array}{cccc}
\widetilde{f}(v, u) & 0 & 0 & 0 \\
0 & \widetilde{g}(v, u) & e^{2 h} & 0 \\
0 & e^{-2 h} & \widetilde{g}(v, u) & 0 \\
0 & 0 & 0 & \widetilde{f}(v, u)
\end{array}\right),
\end{aligned}
$$

где $\widetilde{f}(v, u)$ и $\widetilde{g}(v, u)$ определены в (14). Если положить $h=\omega=\gamma$, то предел

$$
\lim _{\gamma \rightarrow \infty} e^{-2 \gamma} \check{L}(n \mid u)=L_{4 \mathrm{v}}(n \mid u) \equiv\left(\begin{array}{cc}
-u \check{q}_{n} & \sigma_{n}^{-} \\
\sigma_{n}^{+} & u^{-1} \check{q}_{n}
\end{array}\right),
$$


где $\check{q}_{n} \equiv \sigma_{n}^{+} \sigma_{n}^{-}$, дает нам $L$-оператор $L_{4 \mathrm{v}}(n \mid u)$ четырехвершинной модели. Аналогично, предел $\gamma \rightarrow \infty$ преобразует матрицу $e^{-2 \gamma} \check{R}(u, v)$ в $R$-матрицу четырехвершинной модели [35]:

$$
R(u, v)=\left(\begin{array}{cccc}
f(v, u) & 0 & 0 & 0 \\
0 & g(v, u) & 1 & 0 \\
0 & 0 & g(v, u) & 0 \\
0 & 0 & 0 & f(v, u)
\end{array}\right)
$$

где

$$
f(v, u)=\frac{u^{2}}{u^{2}-v^{2}}, \quad g(v, u)=\frac{u v}{u^{2}-v^{2}} .
$$

Матричным элементам $L$-оператора $L_{4 \mathrm{v}}(n \mid u)(17)$ можно поставить в соответствие вершины, изображаемые точками с примыкающими стрелками (рис. 4).

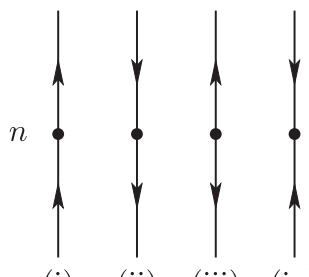

(i) (ii) (iii) (iv)

Рис. 4. Вершинное представление матричных элементов $L$-оператора

Матричный элемент $\left(L_{4 \mathrm{v}}\right)_{11}$ соответствует вершине (i) (рис. 4$)$, где точка отвечает оператору $-u \check{q}_{n}$. Единственное отличное от нуля среднее этого оператора, ${ }_{n}\left\langle\leftarrow\left|-u \check{q}_{n}\right| \leftarrow\right\rangle_{n}$, определяет вершину $b$ (рис. 3) с весом $\omega_{b}=-u$. Элемент $\left(L_{4 \mathrm{v}}\right)_{22}$ соответствует вершине (ii) с точкой, обозначающей оператор $u^{-1} \check{q}_{n}$. Соответствующее ненулевое среднее ${ }_{n}\left\langle\leftarrow\left|u^{-1} \check{q}_{n}\right| \leftarrow\right\rangle_{n}$ определяет вершину $a$ (рис. 3) с ве$\operatorname{com} \omega_{a}=u^{-1}$. Матричные элементы $\left(L_{4 \mathrm{v}}\right)_{12}=\sigma_{n}^{-}$и $\left(L_{4 \mathrm{v}}\right)_{21}=\sigma_{n}^{+}$соответствуют вершинам (iii) и (iv), а ненулевые средние ${ }_{n}\left\langle\rightarrow\left|\sigma_{n}^{-}\right| \leftarrow\right\rangle_{n}$ и ${ }_{n}\left\langle\leftarrow\left|\sigma_{n}^{+}\right| \rightarrow\right\rangle_{n}$ определяют вершины $c$ (рис. 3) с весами, равными единице $\left(\omega_{c}=1\right)$.

Матрища монодромии определяется [70], [71] как матричное произведение $L$-операторов:

$$
T(u)=L(M \mid u) L(M-1 \mid u) \cdots L(0 \mid u)=\left(\begin{array}{ll}
A(u) & B(u) \\
C(u) & D(u)
\end{array}\right) .
$$

Каждому матричному элементу матрицы $T(u)(20)$ поставим в соответствие показанные на рис. 5 вертикальные графы, содержащие $M+1$ узел [35]. Оператору $B(u)$ соответствует граф (B), у которого стрелки на крайних верхнем и нижнем узлах направлены наружу. Оператору $C(u)$ соответствует граф (C), у которого верхняя и нижняя стрелки направлены внутрь. Аналогично, операторам $A(u)$ и $D(u)$ соответствуют графы $(\mathrm{A})$ и $(\mathrm{D})$, у которых направления верхних и нижних стрелок совпадают. При этом аналитическое выражение, 


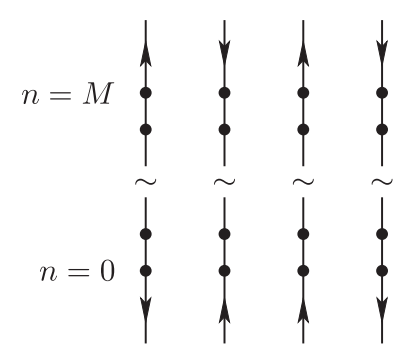

(B) (C) (A) (D)

Рис. 5. Графы, отвечающие матричным элементам матрицы монодромии

например, оператора $B(u)$ дается соотношением

$$
B(u)=\sum_{k_{M}, \ldots, k_{1}=1}^{2}\left(L_{4 \mathrm{v}}\right)_{1 k_{M}}(M \mid u)\left(L_{4 \mathrm{v}}\right)_{k_{M} k_{M-1}}(M-1 \mid u) \cdots\left(L_{4 \mathrm{v}}\right)_{k_{1} 2}(0 \mid u) .
$$

Из (21) видно, что значениям каждого матричного элемента (20) соответствует сумма по всем вариантам расстановки стрелок на одном из графов, показанных на рис. 5. Стрелки расставляются на промежуточных ребрах в соответствии с видом допустимых вершин, показанных на рис. 4.

Перестановочные соотношения элементов матрицы $T(u)(20)$ задаются сплетающим соотношением, содержащим $R$-матрицу (18):

$$
R(v, u)(T(u) \otimes T(v))=(T(v) \otimes T(u)) R(v, u) .
$$

Наиболее важные из соотношений, содержащихся в (22), имеют вид:

$$
\begin{aligned}
C(u) B(v) & =g(v, u)(A(u) D(v)-A(v) D(u)), \\
A(u) B(v) & =f(v, u) B(v) A(u)+g(v, u) B(u) A(v), \\
D(u) B(v) & =f(v, u) B(v) D(u)+g(v, u) B(u) D(v), \\
{[B(u), B(v)] } & =[C(u), C(v)]=0,
\end{aligned}
$$

где $[\cdot, \cdot]$ есть коммутатор матриц. Трансфер-матрица $\tau(u)$ определяется как след матрицы монодромии:

$$
\tau(u)=\operatorname{tr} T(u)=A(u)+D(u) .
$$

Равенство (22) означает, что $[\tau(u), \tau(v)]=0$ при произвольных $u$ и $v$.

Оператор $L_{4 \mathrm{v}}(n \mid u)(17)$ удовлетворяет соотношению

$$
e^{\zeta \sigma_{n}^{z}} L_{4 \mathrm{v}}(n \mid u) e^{-\zeta \sigma_{n}^{z}}=e^{-(\zeta / 2) \sigma^{z}} L_{4 \mathrm{v}}(n \mid u) e^{(\zeta / 2) \sigma^{z}}
$$

где $\zeta$ - произвольный параметр. Из равенства (25) и из определения матрицы монодромии (20) следует, что

$$
e^{\zeta \widehat{S}^{z}} T(u) e^{-\zeta \widehat{S}^{z}}=e^{-\zeta \sigma^{z}} T(u) e^{\zeta \sigma^{z}}, \quad \widehat{S}^{z}=\frac{1}{2} \sum_{l=0}^{M} \sigma_{l}^{z}
$$


где $\widehat{S}^{z}$ является оператором $z$-компоненты полного спина. Равенства

$$
\widehat{S}^{z} B(u)=B(u)\left(\widehat{S}^{z}-1\right), \quad \widehat{S}^{z} C(u)=C(u)\left(\widehat{S}^{z}+1\right)
$$

являются следствием соотношения (26) и означают, что $B(u)$ понижает полный спин системы, а $C(u)$ повышает его. Из $(26)$ следует перестановочность $\tau(u)$ и $\widehat{S}^{z}$ :

$$
\left[\tau(u), \widehat{S}^{z}\right]=0
$$

В квантовом пространстве $\mathfrak{H}_{M+1}$ введем вакуумное состояние $|\Leftarrow\rangle$,

$$
|\Leftarrow\rangle \equiv \bigotimes_{l=0}^{M}|\leftarrow\rangle_{l}=\bigotimes_{l=0}^{M}\left(\begin{array}{l}
1 \\
0
\end{array}\right)_{l}
$$

которое является собственным для операторов $A(u)$ и $D(u)$ :

$$
\begin{array}{ll}
A(u)|\Leftarrow\rangle=\alpha(u)|\Leftarrow\rangle, & \alpha(u)=(-u)^{M+1}, \\
D(u)|\Leftarrow\rangle=\delta(u)|\Leftarrow\rangle, & \delta(u)=\left(u^{-1}\right)^{M+1} .
\end{array}
$$

Кроме того, выполняются соотношения

$$
\langle\Leftarrow|B(u)=0, \quad C(u)| \Leftarrow\rangle=0 .
$$

Основными объектами дальнейшего рассмотрения будут состояние $\left|\Psi_{N}(\mathbf{u})\right\rangle$, полученное последовательным действием $B(u)$ на $|\Leftarrow\rangle$, и соответствующее сопряженное состояние $\left\langle\Psi_{N}(\mathbf{u})\right|$ :

$$
\left|\Psi_{N}(\mathbf{u})\right\rangle=\prod_{i=1}^{N} B\left(u_{i}\right)|\Leftarrow\rangle, \quad\left\langle\Psi_{N}(\mathbf{u})\right|=\langle\Leftarrow| \prod_{j=1}^{N} C\left(u_{j}\right) .
$$

Применяя (27) и (33), получаем полный спин состояния $\left|\Psi_{N}(\mathbf{u})\right\rangle$ :

$$
\widehat{S}^{z}\left|\Psi_{N}(\mathbf{u})\right\rangle=\frac{M+1-2 N}{2}\left|\Psi_{N}(\mathbf{u})\right\rangle .
$$

Из (34) при $N=0$ следует, что полный спин состояния $|\Leftarrow\rangle$ равен $(M+1) / 2$.

Существенную роль в подходе, представляемом в данной работе, будут играть функции Шура $S_{\boldsymbol{\lambda}}\left(\mathbf{u}^{2}\right)$, образующие базис в кольце симметрических многочленов $N$ переменных (доказательство приведено в книге [94]). Для действий над функциями Шура полезно их выражение, задаваемое тождеством Якоби-Труди:

$$
S_{\boldsymbol{\lambda}}\left(\mathbf{u}^{2}\right) \equiv \frac{\operatorname{det}\left(u_{j}^{2\left(\lambda_{k}+N-k\right)}\right)_{1 \leqslant j, k \leqslant N}}{\mathscr{V}_{N}\left(\mathbf{u}^{2}\right)}, \quad \mathscr{V}_{N}\left(\mathbf{u}^{2}\right)=\prod_{1 \leqslant i<k \leqslant N}\left(u_{k}^{2}-u_{i}^{2}\right),
$$

где $\boldsymbol{\lambda}$ - нестрогое разбиение и $\mathscr{V}_{N}\left(\mathbf{u}^{2}\right)$ - определитель Вандермонда (нижний значок $N$ напоминает о “длине" последовательности u). Обобщения тождества Якоби-Труди обсуждаются в [95].

С использованием соотношений (21) и (35) в работе [35] было получено следующее утверждение. 
ПРЕДЛОЖЕНИЕ 1. Вектор состояния $\left|\Psi_{N}(\mathbf{u})\right\rangle$ (33) для четырехвериинной модели приводится к следующему виду:

$$
\begin{aligned}
\left|\Psi_{N}(\mathbf{u})\right\rangle=\left(u_{1} u_{2} \cdots u_{N}\right)^{M-2(N-1)} & \\
& \times \sum_{\widetilde{\boldsymbol{\lambda}} \subseteq\left\{(M-2(N-1))^{N}\right\}}(-1)^{|\widetilde{\boldsymbol{\mu}}|} S_{\widetilde{\boldsymbol{\lambda}}}\left(\mathbf{u}^{-2}\right)\left(\prod_{k=1}^{N} \sigma_{\widetilde{\mu}_{k}}^{-}\right)|\Leftarrow\rangle,
\end{aligned}
$$

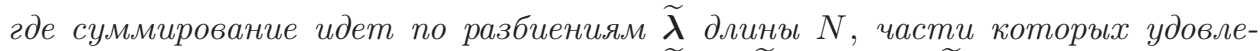
творяют неравенству $M-2(N-1) \geqslant \widetilde{\lambda}_{1} \geqslant \widetilde{\lambda}_{2} \geqslant \cdots \geqslant \widetilde{\lambda}_{N} \geqslant 0, u|\widetilde{\boldsymbol{\mu}}|-$ вес строгого разбиения $\widetilde{\boldsymbol{\mu}}=\widetilde{\boldsymbol{\lambda}}+2 \boldsymbol{\delta}_{N}$. Роль коэфбичиентов в (36) играют функции IIура (35).

Уместно заметить, что в интегрируемой $q$-бозонной модели (см. приложение В, где представлены $q$-бозонная модель и ее комбинаторные аспекты) $L$-оператор аналогичен $L_{4 \mathrm{v}}(n \mid u)(17)$ и, как показано в [61], для $N$-частичного состояния также возникает представление, аналогичное (36).

\section{3. Статистическая сумма четырехвершинной модели и плоские} разбиения. Рассмотрим, следуя [35], четырехвершинную модель с совпадающими статистическими весами третьей и четвертой вершин (рис. 3). Случай, для которого веса $\omega_{a}, \omega_{b}, \omega_{c}$ не зависят от координат узла решетки, называется однородным, и соответствующая статистическая сумма равна

$$
Z\left(\omega_{a}, \omega_{b}, \omega_{c}\right)=\sum \omega_{a}^{l^{a}} \omega_{b}^{l^{b}} \omega_{c}^{l^{c}},
$$

где суммирование ведется по всем допустимым конфигурациям стрелок. При этом число вершин типов $a, b$ и $c$ в каждой конфигурации равно $l^{a}, l^{b}$ и $l^{c}$ coответственно. Допустимые конфигурации зависят от наложенных граничных условий, которые определяются направлением стрелок на границе решетки. Для неоднородного случая веса $\omega_{a}, \omega_{b}, \omega_{c}$ и числа $l^{a}, l^{b}, l^{c}$ приобретают зависимость от координат узлов.

Определим четырехвершинную модель на решетке, состоящей из $2 N \times(M+1)$ узлов ( $M+1$ строка и $2 N$ столбцов), с фиксированными граничными условиями. Пусть стрелки на всех узлах, образующих правую и левую границы решетки, направлены влево. Направления стрелок на всех узлах, образующих верхнюю и нижнюю границу решетки, следующие: для первых $N$ столбцов (считая слева направо) стрелки направлены внутрь, а для остальных $N$ столбцов - наружу (см. рис. 6 для $M=6$ и $N=3$ ).

Для перечисления всех возможных конфигураций стрелок воспользуемся представлением в терминах решеточных путей [35]. Конкретную конфигурацию $N$ решеточных путей будем называть $(N-)$ набором nутей ${ }^{1}$. Пути являются самоизбегающими, т. е. через каждую точку решетки проходит только один из путей, принадлежащих набору. Пусть значения $-N,-N+1, \ldots,-1$ индексируют первые $N$ столбцов решетки, а значения $1,2, \ldots, N$ - остальные столбцы, и пусть номера $n=0$ и $n=M$ отвечают нижней и верхней строкам

\footnotetext{
13аменяя таким образом английский термин "nest of paths".
} 
соответственно. Тогда путь с номером $i, 1 \leqslant i \leqslant N$, идет из узла $(-N+i-1,0)$ в узел $(i, M)$. При этом допустимы звенья путей со стрелками, направленными только вверх или вправо. В вертикальном направлении разрешены несколько последовательных шагов, в горизонтальном - только один шаг. Типичный набор путей изображен на рис. 6. Длина каждого пути в наборе равна $N+M$. Число вершин типа $c$ в допустимом пути равно $2 N$. Число вершин типа $b$ равно $M-N+1$. Это означает, что числа вершин типов $b$ и $c$ в наборе путей равны соответственно $l^{b}=l^{a}=N(M-N+1)$ и $l^{c}=2 N^{2}$.
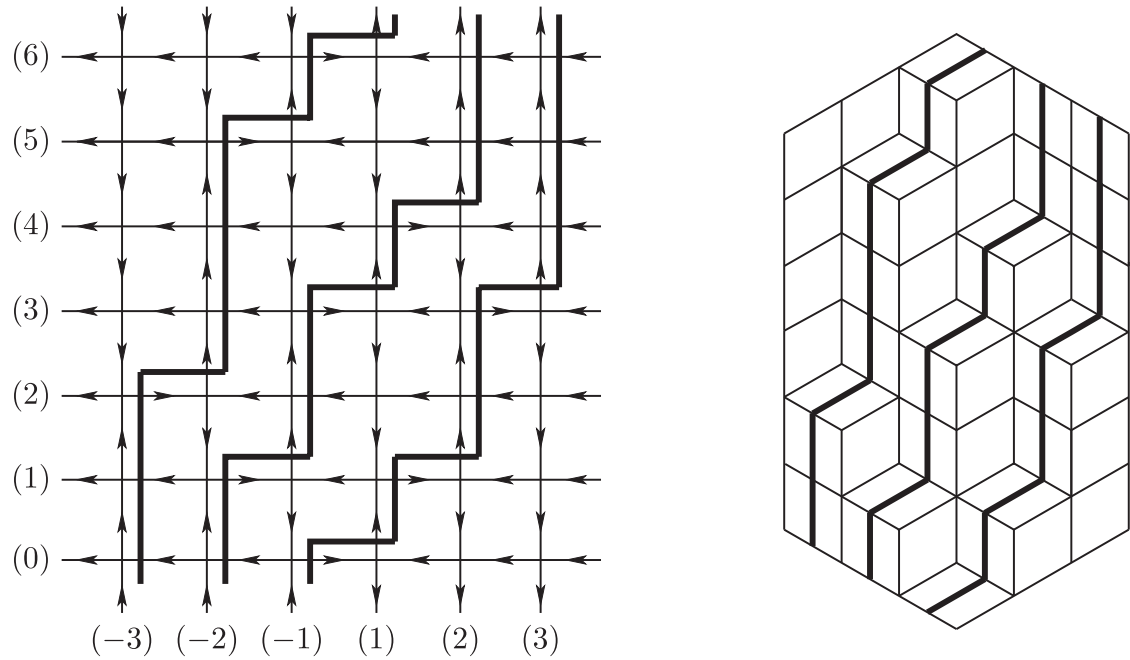

Рис. 6. Типичный набор решеточных путей для четырехвершинной модели с фиксированными граничными условиями и соответствующее строгое плоское разбиение с градиентными линиями

В работе [35] установлено взаимно однозначное соответствие между строгими плоскими разбиениями в $\mathscr{B}(N, N, M)$ и допустимыми наборами самоизбегающих путей на решетке размера $2 N \times(M+1)$ с фиксированными граничными условиями (рис. 6). Каждому допустимому набору путей отвечает матрица $\left(\pi_{i j}\right)_{1 \leqslant i, j \leqslant N}: j$-й путь, отсчитываемый сверху вниз, соответствует $j$-му столбцу матрицы, и элемент $\pi_{i j}$ равен числу клеток в $i$-м столбце решетки (отсчитываемом справа налево) под $j$-м путем. Плоское разбиение в ящике $\mathscr{B}(N, N, M)$ эквивалентно замощению шестиугольника элементарными ромбами с углами $\pi / 3$ и $2 \pi / 3$ (рис. 7). Допустимые замощения ромбами представляют собой проекцию трехмерных диаграмм Юнга с градиентными линиями. На рис. 6 изображены решеточные пути четырехвершинной модели и соответствующие градиентные линии строгого разбиения, характеризующегося $(3 \times 3)$-матрицей

$$
\boldsymbol{\pi}=\left(\begin{array}{lll}
6 & 4 & 3 \\
5 & 3 & 1 \\
2 & 1 & 0
\end{array}\right)
$$




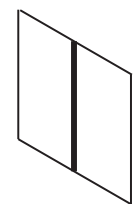

1

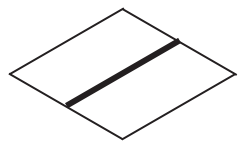

2

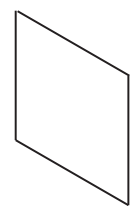

3

Рис. 7. Три типа элементарных ромбов

С учетом сказанного возникает следующее утверждение.

ПреДЛОЖенИЕ 2. Для статистической суммы (37) однородной четырехвериинной модели с фиксированными граничными условиями имеет место представление

$$
Z\left(\omega_{a}, \omega_{b}, \omega_{c}\right)=\left(\omega_{a} \omega_{b}\right)^{N(M-N+1)} \omega_{c}^{2 N^{2}} A(N, N, M-2 N+2) .
$$

ДокАЗАТЕльство. Статистическая сумма указанной модели допускает следующее выражение:

$$
Z\left(\omega_{a}, \omega_{b}, \omega_{c}\right)=\left(\omega_{a} \omega_{b}\right)^{N(M-N+1)} \omega_{c}^{2 N^{2}} S(N, M),
$$

где $S(N, M)$ - число всех допустимых наборов решеточных путей, пронумерованных, как на рис. 6. По построению, $S(N, M)$ в (39) совпадает с числом строгих разбиений $A^{\operatorname{spp}}(N, N, M)(8)$ в ящике $\mathscr{B}(N, N, M)$, которое равно числу плоских разбиений $A(N, N, M-2 N+2)$ в $\mathscr{B}(N, N, M-2 N+2)$ (см. (7) в пределе $q \rightarrow 1)$. В результате для $Z\left(\omega_{a}, \omega_{b}, \omega_{c}\right)(39)$ получаем соотношение $(38)$. Предложение доказано.

Рассмотрим неоднородный случай и допустим для определенности, что веса зависят только от номеров столбцов, образуя $2 N$-элементные последовательности $\boldsymbol{\omega}_{a}, \boldsymbol{\omega}_{b}$ и $\boldsymbol{\omega}_{c}$. Аналогично, вместо чисел $l^{a}, l^{b}$ и $l^{c}$ возникают $2 N$-элементные последовательности $\mathbf{l}^{a}, \mathbf{l}^{b}$ и $\mathbf{l}^{c}$ соответственно. С учетом принятой нумерации столбцов (рис. 6) имеем, например, $\mathbf{l}^{a}=\left(l_{-N}^{a}, l_{-N+1}^{a}, \ldots, l_{-1}^{a}, l_{1}^{a}, \ldots, l_{N}^{a}\right)$ и т. д. Тогда статистическая сумма (37) принимает вид

$$
Z\left(\boldsymbol{\omega}_{a}, \boldsymbol{\omega}_{b}, \boldsymbol{\omega}_{c}\right)=\sum \prod_{i=-1}^{-N}\left(\boldsymbol{\omega}_{a}\right)_{i}^{l_{i}^{a}}\left(\boldsymbol{\omega}_{b}\right)_{i}^{l_{i}^{b}}\left(\boldsymbol{\omega}_{c}\right)_{i}^{l_{i}^{c}} \prod_{j=1}^{N}\left(\boldsymbol{\omega}_{a}\right)_{j}^{l_{j}^{a}}\left(\boldsymbol{\omega}_{b}\right)_{j}^{l_{j}^{b}}\left(\boldsymbol{\omega}_{c}\right)_{j}^{l_{j}^{c}}
$$

Конкретизируем вершинные веса следующим образом:

$$
\begin{array}{lllr}
\left(\boldsymbol{\omega}_{a}\right)_{i}=v_{-i}^{-1}, & \left(\boldsymbol{\omega}_{b}\right)_{i}=v_{-i}, & \left(\boldsymbol{\omega}_{c}\right)_{i}=1, & -N \leqslant i \leqslant-1, \\
\left(\boldsymbol{\omega}_{a}\right)_{j}=u_{j}^{-1}, & \left(\boldsymbol{\omega}_{b}\right)_{j}=u_{j}, & \left(\boldsymbol{\omega}_{c}\right)_{j}=1, & 1 \leqslant j \leqslant N .
\end{array}
$$

Представляет интерес рассмотреть веса (41) в $q$-параметризации:

$$
\mathbf{v}^{-2}=\mathbf{q} \equiv\left(q, q^{2}, \ldots, q^{N}\right), \quad \mathbf{u}^{2}=\frac{\mathbf{q}}{q} \equiv\left(1, q, \ldots, q^{N-1}\right) .
$$

Тогда имеет место следующее утверждение. 
ПреДЛОжЕНИЕ 3. Статистическая сумма (40) неоднородной четырехвершинной модели с фиксированными граничными условиями в q-параметризаиии, определяемой соотношениями (41) и (42), приводится к виду

$$
\left.Z\left(\boldsymbol{\omega}_{a}, \boldsymbol{\omega}_{b}, \boldsymbol{\omega}_{c}\right)\right|_{q-\text {-парам. }}=q^{-N^{2} M / 2} Z_{q}^{\mathrm{spp}}(N, N, M),
$$

где $Z_{q}^{\mathrm{spp}}(N, N, M)$ - производящая функция (6) строгих плоских разбиений в $\mathscr{B}(N, N, M)$.

ДокАзАтЕльство. Соотношение (40) в обозначениях (41) принимает вид

$$
\left.Z\left(\boldsymbol{\omega}_{a}, \boldsymbol{\omega}_{b}, \boldsymbol{\omega}_{c}\right)\right|_{(\mathbf{u}-\mathbf{v}) \text {-парам. }}=\sum \prod_{i=-1}^{-N} v_{-i}^{l_{i}^{b}-l_{i}^{a}} \prod_{j=1}^{N} u_{j}^{l_{j}^{b}-l_{j}^{a}},
$$

где суммирование ведется по всем наборам решеточных путей. Представление (44) в $q$-параметризации (42) принимает вид

$$
\left.Z\left(\boldsymbol{\omega}_{a}, \boldsymbol{\omega}_{b}, \boldsymbol{\omega}_{c}\right)\right|_{q \text {-парам. }}=\sum q^{(1 / 2) \sum_{i=-1}^{-N} i\left(l_{i}^{a}-l_{i}^{b}\right)+(1 / 2) \sum_{j=1}^{N}(j-1)\left(l_{j}^{a}-l_{j}^{b}\right)} .
$$

С другой стороны, в работе [62] для объема трехмерной строгой диаграммы Юнга в $\mathscr{B}(N, N, M)$ была получена формула

$$
\left|\boldsymbol{\pi}_{\mathrm{spp}}\right|=\frac{N^{2} M}{2}+\frac{1}{2} \sum_{i=-1}^{-N} i\left(l_{i}^{a}-l_{i}^{b}\right)+\frac{1}{2} \sum_{j=1}^{N}(j-1)\left(l_{j}^{a}-l_{j}^{b}\right) .
$$

С использованием этой формулы выражение (45) приводится к виду

$$
\left.Z\left(\boldsymbol{\omega}_{a}, \boldsymbol{\omega}_{b}, \boldsymbol{\omega}_{c}\right)\right|_{q \text {-парам. }}=q^{-N^{2} M / 2} \sum_{\left\{\boldsymbol{\pi}_{\mathrm{spp}}\right\}} q^{\left|\boldsymbol{\pi}_{\mathrm{spp}}\right|},
$$

где суммирование ведется по всем строгим плоским разбиениям в $\mathscr{B}(N, N, M)$. Другими словами, мы приходим к соотношению (43), согласующемуся при $q \rightarrow 1$ с ответом для однородной модели (38). Предложение доказано.

Применение КМОЗ позволяет исследовать четырехвершинную модель на решетке конечного размера с различными граничными условиями [35]: статистическая сумма модели с фиксированными граничными условиями имеет смысл производящей функции строгих плоских разбиений; в случае периодических граничных условий четырехвершинная модель эквивалентна модели покрытия тора элементарными ромбами. В частности, справедливо следующее утверждение.

ПРЕДЛОЖЕНИЕ 4. Статистическая сумма (44) неоднородной четырехвершинной модели с фиксированными граничными условиями в параметризации, определяемой соотношениями (41), (42), имеет следующий вид:

$$
\begin{gathered}
\left.Z\left(\boldsymbol{\omega}_{a}, \boldsymbol{\omega}_{b}, \boldsymbol{\omega}_{c}\right)\right|_{(\mathbf{u}-\mathbf{v}) \text {-nарам. }}=\operatorname{det}\left(H_{i j}\right)_{1 \leqslant i, j \leqslant N} \\
\quad \times \prod_{1 \leqslant i_{1}<j_{1} \leqslant N} \frac{v_{i_{1}} v_{j_{1}}}{v_{i_{1}}^{2}-v_{j_{1}}^{2}} \prod_{1 \leqslant i_{2}<j_{2} \leqslant N} \frac{u_{i_{2}} u_{j_{2}}}{u_{j_{2}}^{2}-u_{i_{2}}^{2}} .
\end{gathered}
$$


Матричные элементы $H_{i j}$ определены следуюшим образом:

$$
H_{i j}=\left(\frac{v_{i}}{u_{j}}\right)^{M-N+1} h_{M-N+2}\left(u_{j}^{2} v_{i}^{-2}\right), \quad h_{P}(x) \equiv \frac{1-x^{P}}{1-x} \quad(P \in \mathbb{N}) .
$$

ДоказАтельство. Рассмотрим скалярное произведение векторов состояния (33):

$$
W(\mathbf{u} ; \mathbf{v}) \equiv\left\langle\Psi_{N}(\mathbf{v}) \mid \Psi_{N}(\mathbf{u})\right\rangle=\left\langle\Leftarrow\left|\prod_{i=1}^{N} C\left(v_{i}\right) \prod_{j=1}^{N} B\left(u_{j}\right)\right| \Leftarrow\right\rangle,
$$

где $\mathbf{u}$ и $\mathbf{v}$ - независимые параметры. Матричные элементы (48) связаны с двумерной решеткой размера $2 N \times(M+1)$. Первые $N$ вертикальных линий решетки ассоциируются с операторами $C\left(v_{i}\right)$, а $N$ последних - с операторами $B\left(u_{j}\right)$. Из графического представления операторов $B(u)$ и $C(u)$ (см. рис. 4,5$)$ следует, что $W(\mathbf{u} ; \mathbf{v})(48)$ сводится к сумме по всем конфигурациям путей на решетке с фиксированными граничными условиями (рис. 6):

$$
W(\mathbf{u} ; \mathbf{v})=(-1)^{M N} \sum \prod_{i=-1}^{-N} v_{-i}^{l_{i}^{b}-l_{i}^{a}} \prod_{j=1}^{N} u_{j}^{l_{j}^{b}-l_{j}^{a}}
$$

Сравнивая (49) с (44), получаем статистическую сумму неоднородной четырехвершинной модели в виде

$$
Z\left(\boldsymbol{\omega}_{a}, \boldsymbol{\omega}_{b}, \boldsymbol{\omega}_{c}\right)=(-1)^{M N} W(\mathbf{u} ; \mathbf{v})
$$

Перестановочные соотношения (23) позволяют вычислить скалярное произведение (48) для произвольной параметризации u, v [35], [62], [70], [71]. В результате с учетом (50) приходим к соотношениям (46), (47), которые и дают статистическую сумму неоднородной модели в виде определителя. Предложение доказано.

В параметризации (42) получаем из (50) следующее равенство:

$$
\left.Z\left(\boldsymbol{\omega}_{a}, \boldsymbol{\omega}_{b}, \boldsymbol{\omega}_{c}\right)\right|_{q \text {-парам. }}=(-1)^{N M} W\left(\mathbf{q}^{-1 / 2} ;\left(\frac{\mathbf{q}}{q}\right)^{1 / 2}\right),
$$

и приходим к соотношению

$$
W\left(\mathbf{q}^{-1 / 2} ;\left(\frac{\mathbf{q}}{q}\right)^{1 / 2}\right)=(-1)^{N M+N(N-1) / 2} \operatorname{det} Q \prod_{1 \leqslant k<j \leqslant N}\left(q^{(j-k) / 2}-q^{-(j-k) / 2}\right)^{-2}
$$

где элементы матрицы $Q=\left(Q_{i j}\right)_{1 \leqslant i, j \leqslant N}$ заданы формулой $(47)$ при $u_{j}^{2} v_{i}^{-2}=$ $q^{i+j-1}$. Определитель матрицы $Q$ вычисляется с помощью соотношения, полученного в [28]:

$$
\operatorname{det}\left(\frac{1-s^{j+k-1}}{1-q^{j+k-1}}\right)_{1 \leqslant j, k \leqslant N}=\mathscr{V}_{N}(\mathbf{q}) \mathscr{V}_{N}\left(\frac{\mathbf{q}}{q}\right) \prod_{k=1}^{N} \prod_{j=1}^{N} \frac{1-s q^{j-k}}{1-q^{j+k-1}}
$$


В результате (52) принимает вид

$$
\begin{aligned}
W\left(\mathbf{q}^{-1 / 2} ;\left(\frac{\mathbf{q}}{q}\right)^{1 / 2}\right) & =(-1)^{N M} q^{-N^{2}(M+2-2 N) / 2} \prod_{k=1}^{N} \prod_{j=1}^{N} \frac{1-q^{M+3-j-k}}{1-q^{k+j-1}} \\
& =(-1)^{N M} q^{-N^{2} M / 2} Z_{q}^{\text {spp }}(N, N, M),
\end{aligned}
$$

откуда видно, что статистическая сумма (51) совпадает с (43).

Как показано в [62], использование вектора состояния в форме (36) позволяет получить для статистической суммы представление (46) в виде определителя. При этом используется формула Бине-Коши [96], записанная в терминах функций Шура (35). При переходе к $q$-параметризации именно на этом пути выявляется связь рассматриваемых корреляционных функций с производящими функциями плоских разбиений и самоизбегающих решеточных путей. В оставшейся части работы будет продемонстрировано, что аналогичные комбинаторные проявления возникают и для корреляционных функций спиновой $X X Z$-цепочки Гейзенберга в пределах нулевой и бесконечной анизотропии.

\section{3. Гамильтониан $X X Z$-магнетика, векторы состояния и функции Шура}

Перейдем к комбинаторике, связанной с корреляционными функциями $X X Z$ магнетика Гейзенберга в пределах нулевой и бесконечной анизотропии. Начнем с общих положений, касающихся $X X Z$-модели при произвольной анизотропии.

3.1. Произвольная анизотропия. Гамильтониан $X X Z$-модели на одномерной решетке из $M+1$ узлов, $M+1=0(\bmod 2)$, пронумерованных элементами множества $\mathbf{M} \equiv\{0 \leqslant l \leqslant M \mid l \in \overline{\mathbb{N}}\}$, имеет вид

$$
\widehat{H}_{X X Z}=-\frac{1}{2} \sum_{k=0}^{M}\left(\sigma_{k+1}^{-} \sigma_{k}^{+}+\sigma_{k+1}^{+} \sigma_{k}^{-}+\frac{\Delta}{2}\left(\sigma_{k+1}^{z} \sigma_{k}^{z}-\mathbb{I}\right)+h \sigma_{k}^{z}\right),
$$

где $\Delta \in \mathbb{R}$ - внутренняя анизотропия, $h \in \mathbb{R}$ - магнитное поле. Локальные спиновые операторы $\sigma_{k}^{ \pm}=\left(\sigma_{k}^{x} \pm i \sigma_{k}^{y}\right) / 2$ и $\sigma_{k}^{z}$, зависящие от $k \in \mathbf{M}$, определены в (9) и действуют над пространством состояний $\mathfrak{H}_{M+1}=\bigotimes_{k=0}^{M} \mathfrak{h}_{k}$, где $\mathfrak{h}_{k} \equiv \mathbb{C}^{2}$. Пространство $\mathfrak{H}_{M+1}$ натянуто на векторы состояния $\bigotimes_{k=0}^{M}|s\rangle_{k}$, где $|s\rangle_{k}$ означает состояния со спином либо "вверх", $|\uparrow\rangle$, либо “вниз", $|\downarrow\rangle$, на $k$-м узле. Состояния $|\uparrow\rangle \equiv\left(\begin{array}{l}1 \\ 0\end{array}\right)$ и $|\downarrow\rangle \equiv\left(\begin{array}{l}0 \\ 1\end{array}\right)$ образуют естественный базис линейного пространства $\mathbb{C}^{2}$. Цепочка считается замкнутой, и наложены периодические граничные условия: $\sigma_{k+(M+1)}^{\#}=\sigma_{k}^{\#}$. Гамильтониан $(54)$ перестановочен с оператором третьей компоненты полного спина $\widehat{S}^{z}$ :

$$
\left[\widehat{H}_{X X Z}, \widehat{S}^{z}\right]=0, \quad \widehat{S}^{z} \equiv \frac{1}{2} \sum_{k=0}^{M} \sigma_{k}^{z}
$$


Рассмотрим произвольное состояние на цепочке, характеризующееся тем, что $N$ узлов заняты спинами "вниз" и $M-N+1$ узлов - спинами "вверх". Пусть параметры $\mu_{i}, 1 \leqslant i \leqslant N$, нумеруют узлы, занятые спинами "вниз", и образуют строгое разбиение $\boldsymbol{\mu}=\left(\mu_{1}, \mu_{2}, \ldots, \mu_{N}\right)$, где $M \geqslant \mu_{1}>\mu_{2}>\cdots>$ $\mu_{N} \geqslant 0$. Вектор состояния для $N$ спинов "вниз", $\left|\Psi_{N}\left(u_{1}, \ldots, u_{N}\right)\right\rangle$, определяется следующим образом:

$$
\left|\Psi_{N}(\mathbf{u})\right\rangle=\sum_{\boldsymbol{\mu}} \chi_{\boldsymbol{\mu}}^{X X Z}(\mathbf{u})\left(\prod_{k=1}^{N} \sigma_{\mu_{k}}^{-}\right)|\Uparrow\rangle, \quad|\Uparrow\rangle \equiv \bigotimes_{n=0}^{M}|\uparrow\rangle_{n},
$$

где суммирование идет по всем разбиениям $\boldsymbol{\mu}$, образующим множество из $C_{M}^{N} \equiv$ $M ! /(N !(M-N) !)$ элементов. Здесь состояние $|\Uparrow\rangle$ отвечает спинам "вверх" на всех узлах. Векторы состояния $\left(\prod_{k=1}^{N} \sigma_{\mu_{k}}^{-}\right)|\uparrow\rangle$ отвечают $N$ спинам “вниз", так как каждый из операторов $\sigma_{\mu_{k}}^{-}$действует на $|\uparrow\rangle$, преобразуя спин "вверх" на узле $\mu_{k}$ в состояние спин "вниз". Коэффициентная функция $\chi_{\boldsymbol{\mu}}^{X X Z}(\mathbf{u})$ в (55) имеет вид

$$
\chi_{\boldsymbol{\mu}}^{X X Z}(\mathbf{u})=\sum_{S_{p_{1}, p_{2}, \ldots, p_{N}}} \mathscr{A}_{S}(\mathbf{u}) u_{p_{1}}^{2 \mu_{1}} u_{p_{2}}^{2 \mu_{2}} \cdots u_{p_{N}}^{2 \mu_{N}}
$$

где суммирование распространяется на все элементы симметрической группы перестановок $S_{p_{1}, p_{2}, \ldots, p_{N}} \equiv S\left(\begin{array}{cccc}1, & 2, & \ldots, & N \\ p_{1}, & p_{2}, & \ldots, & p_{N}\end{array}\right)$. Амплитуда $\mathscr{A}_{S}(\mathbf{u})(56)$ имеет вид

$$
\mathscr{A}_{S}(\mathbf{u}) \equiv \prod_{1 \leqslant j<i \leqslant N} \frac{1-2 \Delta u_{p_{i}}^{2}+u_{p_{i}}^{2} u_{p_{j}}^{2}}{u_{p_{i}}^{2}-u_{p_{j}}^{2}}
$$

Состояние $\left|\Psi_{N}(\mathbf{u})\right\rangle$ (55) определено аналогично (36).

Векторы состояния (55) являются собственными для гамильтониана (54),

$$
\widehat{H}_{X X Z}\left|\Psi_{N}(\mathbf{u})\right\rangle=E_{N}(\mathbf{u})\left|\Psi_{N}(\mathbf{u})\right\rangle,
$$

тогда и только тогда, когда параметры $u_{l}(1 \leqslant l \leqslant N)$ удовлетворяют уравнениям Бете:

$$
u_{l}^{2(M+1)}=(-1)^{N-1} \prod_{k=1}^{N} \frac{1-2 \Delta u_{l}^{2}+u_{l}^{2} u_{k}^{2}}{1-2 \Delta u_{k}^{2}+u_{l}^{2} u_{k}^{2}}
$$

Соответствующие собственные значения энергии имеют вид

$$
E_{N}^{X X Z}(\mathbf{u})=-\frac{1}{2} \sum_{i=1}^{N}\left(u_{i}^{2}+u_{i}^{-2}-2 \Delta-2 h\right) .
$$

Будем рассматривать $X X Z$-модель для двух значений анизотропии: $\Delta=0$ и $\Delta \rightarrow-\infty$ [80], [97], [98]. 
3.2. Нулевая анизотропия ( $X X 0$-магнетик). Гамильтониан модели $X X 0(\equiv X X)$ описывает взаимодействие размещенных на ближайших узлах периодической цепочки спинов "вверх" и "вниз"; в нулевом магнитном поле он имеет вид

$$
\widehat{H}_{X X} \equiv-\frac{1}{2} \sum_{k=0}^{M} \widehat{h}_{k+1, k}, \quad \widehat{h}_{k+1, k} \equiv \sigma_{k+1}^{-} \sigma_{k}^{+}+\sigma_{k+1}^{+} \sigma_{k}^{-} .
$$

Система, описываемая (60), эквивалентна свободным фермионам [54] и представляет интерес, например, для описания экситонов Френкеля [85], а также в связи с построением теории квантовых вычислений [86], [99].

Для получения вектора состояния $\left|\Psi_{N}(\mathbf{u})\right\rangle$ и уравнений Бете $X X$-модели выполняется переход $\Delta \rightarrow 0$ в соотношениях (57) и (58). В результате получаем, что гамильтониан (60) диагонализуется на векторах состояния (55) с коэффициентной функцией следующего вида:

$$
\chi_{\boldsymbol{\mu}}^{X X}(\mathbf{u})=\operatorname{det}\left(u_{j}^{2 \mu_{k}}\right)_{1 \leqslant j, k \leqslant N} \prod_{1 \leqslant n<l \leqslant N}\left(u_{l}^{2}-u_{n}^{2}\right)^{-1} .
$$

При этом уравнения Бете в параметризации $u_{j}^{2}=e^{i \theta_{j}}$ возникают в виде (см. [83])

$$
e^{i(M+1) \theta_{j}}=(-1)^{N-1}, \quad 1 \leqslant j \leqslant N .
$$

Соответствующие решения

$$
\theta_{j}=\frac{2 \pi}{M+1}\left(I_{j}-\frac{N-1}{2}\right)
$$

параметризуются числами $I_{j}$, которые могут быть целыми или полуцелыми в зависимости от того, является $N$ четным или нечетным. Достаточно рассматривать $N$ чисел $I_{j}$, удовлетворяющих условию $M \geqslant I_{1}>I_{2}>\cdots>I_{N} \geqslant 0$.

Обозначение $\boldsymbol{\theta}$ для $N$-элементной совокупности $\left(\theta_{1}, \theta_{2}, \ldots, \theta_{N}\right)$ решений (63) будет в дальнейшем подчеркивать использование именно решения уравнения Бете. При этом $e^{i \boldsymbol{\theta}} \equiv\left(e^{i \theta_{1}}, e^{i \theta_{2}}, \ldots, e^{i \theta_{N}}\right)$. В противном случае использование обозначения $\mathbf{u} \equiv\left(u_{1}, u_{2}, \ldots, u_{N}\right)$ или $\mathbf{u}^{2} \equiv\left(u_{1}^{2}, u_{2}^{2}, \ldots, u_{N}^{2}\right)$ указывает на то, что имеется в виду набор произвольных параметров.

Собственные значения энергии (59) для $X X$-модели имеют вид

$$
E_{N}^{X X}(\boldsymbol{\theta})=-\sum_{j=1}^{N} \cos \theta_{j}=-\sum_{j=1}^{N} \cos \left(\frac{2 \pi}{M+1}\left(I_{j}-\frac{N-1}{2}\right)\right) .
$$

Для энергии основного состояния $E_{N}^{X X}\left(\boldsymbol{\theta}^{\text {g }}\right)$ используем (63) при параметризации $I_{j}=N-j$ :

$$
\begin{aligned}
E_{N}^{X X}\left(\boldsymbol{\theta}^{\mathrm{g}}\right) & =-\sin \frac{\pi N}{M+1} \operatorname{cosec} \frac{\pi}{M+1}, \\
\theta_{j}^{\mathrm{g}} & \equiv \frac{2 \pi}{M+1}\left(\frac{N+1}{2}-j\right), \quad 1 \leqslant j \leqslant N .
\end{aligned}
$$


3.3. Бесконечная анизотропия (изинговский магнетик). Менее изученным пределом $X X Z$-модели является предел сильной анизотропии $\Delta \rightarrow-\infty$ (аббревиатура SA) [23], [66], [100], [101]. Этот предел уместно называть изинговским, так как простейший гамильтониан в этом пределе оказывается гамильтонианом одномерной модели Изинга [23]:

$$
\lim _{\Delta \rightarrow-\infty} \frac{1}{\Delta} \widehat{H}_{X X Z}=\widehat{H}_{\mathrm{IZ}} \equiv \frac{-1}{4} \sum_{k=0}^{M}\left(\sigma_{k+1}^{z} \sigma_{k}^{z}-\mathbb{I}\right) .
$$

Однако, следуя [98], мы будем рассматривать случай сильной анизотропии с использованием эффективного гамильтониана $\widehat{H}_{\mathrm{SA}}$, который формально эквивалентен $X X$-гамильтониану (60), но для которого учитывается требование, запрещающее двум состояниям со спинами “вниз" занимать какую-либо пару ближайших узлов [100], [101]:

$$
\widehat{H}_{\mathrm{SA}}=-\frac{1}{2} \sum_{k=0}^{M} \mathrm{P}\left(\widehat{h}_{k+1, k}+h \sigma_{k}^{z}\right) \mathrm{P}, \quad \mathrm{P} \equiv \prod_{k=0}^{M}\left(\mathbb{I}-\hat{q}_{k+1} \hat{q}_{k}\right) .
$$

Проекторы $\mathrm{P}$ в $\widehat{H}_{\mathrm{SA}}(67)$ определены так, чтобы обращать в нуль состояния со спинами “вниз" для любой пары ближайших узлов. При этом локальные проекторы на состояния со спинами "вниз" и "вверх" зависят от решеточного аргумента $k \in \mathbf{M}$ (обобщается определение $(11))$ и имеют вид

$$
\hat{q}_{k} \equiv \frac{1}{2}\left(\mathbb{I}-\sigma_{k}^{z}\right), \quad \check{q}_{k} \equiv \frac{1}{2}\left(\mathbb{I}+\sigma_{k}^{z}\right), \quad \check{q}_{k}+\hat{q}_{k}=\mathbb{I}, \quad k \in \mathbf{M}
$$

Гамильтонианы (66) и (67) перестановочны. Действительно, с помощью (68) можно представить $\widehat{H}_{\mathrm{IZ}}(66)$ в следующем виде:

$$
\widehat{H}_{\mathrm{IZ}}=\widehat{N}-\sum_{k=0}^{M} \widehat{q}_{k+1} \hat{q}_{k}, \quad \widehat{N} \equiv \sum_{k=0}^{M} \hat{q}_{k},
$$

где оператор числа частиц $\widehat{N}$ перестановочен с гамильтонианами $\widehat{H}_{X X}$ и $\widehat{H}_{\mathrm{SA}}$. В силу определения проектора $\mathrm{P}$ соблюдается соотношение

$$
\mathrm{P} \sum_{k=0}^{M} \hat{q}_{k+1} \hat{q}_{k}=\sum_{k=0}^{M} \hat{q}_{k+1} \hat{q}_{k} \mathrm{P}=0 .
$$

Следовательно, гамильтонианы (66) и (67) также перестановочны: $\left[\widehat{H}_{\mathrm{SA}}, \widehat{H}_{\mathrm{IZ}}\right]=0$, и поэтому обладают общей системой собственных функций.

Волновая функция (56) в пределе $\Delta \rightarrow-\infty$ принимает вид

$$
\chi_{\boldsymbol{\mu}}^{\mathrm{SA}}(\mathbf{u})=\operatorname{det}\left(u_{j}^{2\left(\mu_{k}-N+k\right)}\right)_{1 \leqslant j, k \leqslant N} \prod_{1 \leqslant n<l \leqslant N}\left(u_{l}^{2}-u_{n}^{2}\right)^{-1},
$$

где координаты состояний со спинами "вниз" образуют, как и в (61), строгое разбиение $\boldsymbol{\mu}$, т. е. $M \geqslant \mu_{1}>\mu_{2}>\cdots>\mu_{N} \geqslant 0$. Кроме того, из (69) следует, 
что волновая функция отлична от нуля тогда и только тогда, когда части $\mu_{i}$, $1 \leqslant i \leqslant N$, удовлетворяют условию исключения: $\mu_{i}>\mu_{i+1}+1$. Таким образом, наличие двух спинов "вниз" на ближайших узлах запрещено. Уравнения Бете в случае $\Delta \rightarrow-\infty$ принимают вид

$$
e^{i(M+1-N) \theta_{k}}=(-1)^{N-1} \prod_{j=1}^{N} e^{-i \theta_{j}}, \quad 1 \leqslant k \leqslant N
$$

и имеют решения

$$
\theta_{j}=\frac{2 \pi}{M+1-N}\left(I_{j}-\frac{N-1}{2}-P\right)
$$

где $P \equiv \frac{1}{2 \pi} \sum_{j=1}^{N} \theta_{j}$. В зависимости от того, четно или нечетно $N$, числа $I_{j}$ являются целыми или полуцелыми, они удовлетворяют неравенству $M-N \geqslant$ $I_{1}>I_{2}>\cdots>I_{N} \geqslant 0$. Основное состояние определяется решением при $P=0$ :

$$
\theta_{j}^{\mathrm{g}}=\frac{2 \pi}{M+1-N}\left(N-j-\frac{N-1}{2}\right) .
$$

Собственные значения энергии имеют вид

$$
E_{N}^{\mathrm{SA}}(\boldsymbol{\theta})=-\sum_{j=1}^{N} \cos \left(\frac{2 \pi}{M+1-N}\left(I_{j}-\frac{N-1}{2}-P\right)\right) .
$$

Собственная энергия основного состояния, вычисленная с помощью (59) и (72), такова:

$$
E_{N}^{\mathrm{SA}}\left(\boldsymbol{\theta}^{\mathrm{g}}\right)=h N-\sin \frac{\pi N}{M+1-N} \operatorname{cosec} \frac{\pi}{M+1-N} .
$$

Собственная энергия изинговского гамильтониана (66) равна $E_{\mathrm{IZ}}=N$.

Оба случая, описываемые гамильтонианами (60) и (67), можно объединить, так как $N$-частичные состояния $\left|\Psi_{N}(\mathbf{u})\right\rangle$ допускают представления, аналогичные представлению (36) (см. [80] [97], [98]). С учетом предельных выражений (61) и (69) для коэффициентной функции (56) вектор состояния (55) принимает следующий вид (с функциями Шура $S_{\boldsymbol{\lambda}}\left(\mathbf{u}^{2}\right)(35)$ в качестве коэффициентов).

(*) Нулевая анизотропия:

$$
\left|\Psi_{N}(\mathbf{u})\right\rangle=\sum_{\boldsymbol{\lambda} \subseteq\left\{\mathscr{M}^{N}\right\}} S_{\boldsymbol{\lambda}}\left(\mathbf{u}^{2}\right)\left(\prod_{k=1}^{N} \sigma_{\mu_{k}}^{-}\right)|\uparrow\rangle,
$$

где суммирование ведется по нестрогим разбиениям $\boldsymbol{\lambda}=\boldsymbol{\mu}-\boldsymbol{\delta}_{N}, \boldsymbol{\delta}_{N}=(N-1$, $N-2, \ldots, 1,0)$, части которых удовлетворяют неравенству $\mathscr{M} \equiv M+1-N \geqslant$ $\lambda_{1} \geqslant \lambda_{2} \geqslant \cdots \geqslant \lambda_{N} \geqslant 0$.

$(* *)$ Бесконечная анизотропия:

$$
\left|\Psi_{N}(\mathbf{u})\right\rangle=\sum_{\tilde{\boldsymbol{\lambda}} \subseteq\left\{(M-2(N-1))^{N}\right\}} S_{\widetilde{\boldsymbol{\lambda}}}\left(\mathbf{u}^{2}\right)\left(\prod_{k=1}^{N} \sigma_{\widetilde{\mu}_{k}}^{-}\right)|\Uparrow\rangle,
$$


где суммирование ведется по разбиениям $\widetilde{\boldsymbol{\lambda}}=\widetilde{\boldsymbol{\mu}}-2 \boldsymbol{\delta}_{N}$, части которых удовлетворяют неравенству $M+2(1-N) \geqslant \widetilde{\lambda}_{1} \geqslant \widetilde{\lambda}_{2} \geqslant \cdots \geqslant \widetilde{\lambda}_{N} \geqslant 0$. В случае бесконечной анизотропии части строгих разбиений $\widetilde{\boldsymbol{\mu}}$ удовлетворяют условию $\widetilde{\mu}_{i}>\widetilde{\mu}_{i+1}+1$.

Векторы состояния, сопряженные векторам (75) и (76), возникают из соотношений, аналогичных (33). Например, состояние $\left\langle\Psi_{N}(\mathbf{v})\right|$, которое сопряжено (75), принимает вид

$$
\left\langle\Psi_{N}(\mathbf{v})\right|=\sum_{\boldsymbol{\lambda} \subseteq\left\{\mathscr{M}^{N}\right\}}\langle\Uparrow|\left(\prod_{k=1}^{N} \sigma_{\mu_{k}}^{+}\right) S_{\boldsymbol{\lambda}}\left(\mathbf{v}^{-2}\right) .
$$

Использование представлений (75), (76) и (77) позволяет применять теорию симметрических функций [52] для вычисления форм-факторов и температурных корреляционных функций. При этом формула Бине-Коши [96], записанная с помощью функций Шура, зависящих от наборов аргументов у и х, принимает вид

$$
\mathscr{P}_{L / n}(\mathbf{y}, \mathbf{x}) \equiv \sum_{\boldsymbol{\lambda} \subseteq\left\{(L / n)^{N}\right\}} S_{\boldsymbol{\lambda}}(\mathbf{y}) S_{\boldsymbol{\lambda}}(\mathbf{x})=\left(\prod_{l=1}^{N} y_{l}^{n} x_{l}^{n}\right) \frac{\operatorname{det}\left(T_{k j}\right)_{1 \leqslant k, j \leqslant N}}{\mathscr{V}_{N}(\mathbf{y}) \mathscr{V}_{N}(\mathbf{x})}
$$

где сумма $\sum_{\boldsymbol{\lambda} \subseteq\left\{(L / n)^{N}\right\}}$ берется по области, охватывающей все разбиения $\boldsymbol{\lambda}$, части которых подчиняются неравенству $L \geqslant \lambda_{1} \geqslant \lambda_{2} \geqslant \cdots \geqslant \lambda_{N} \geqslant n$, где $n \geqslant 0$. В соотношении (78) используются определители Вандермонда $\mathscr{V}_{N}(35)$, а матричные элементы $T_{k j}$ имеют вид

$$
T_{k j}=\frac{1-\left(x_{k} y_{j}\right)^{N+L-n}}{1-x_{k} y_{j}} .
$$

\section{4. О выборе эффективного гамильтониана при бесконечной ани-} зотропии. В работе [102] показано, что трансфер-матрица шестивершинной модели (см. (24)) перестановочна с гамильтонианом $X X Z$-модели (54): $\left[\widehat{H}_{X X Z}, \tau_{6 \mathrm{v}}(u)\right]=0$.

Далее, имеет место следующая теорема [98].

ТЕОРема 1. Трансфер-матрица четырехвериинной модели $\tau_{4 \mathrm{v}}(u)$ перестановочна с эффективным гамильтонианом $\widehat{H}_{\mathrm{SA}}(67)$ :

$$
\left[\widehat{H}_{\mathrm{SA}}, \tau_{4 \mathrm{v}}(u)\right]=0 .
$$

ДоказАтеЛьство. Шаг I. Введем оператор $\mathscr{C}_{n+1, n} \equiv \mathbb{I}-\hat{q}_{n+1} \hat{q}_{n}$ (см. (68)) и запишем проектор Р, определенный в (67), в виде Р $\equiv \prod_{n=0}^{M} \mathscr{C}_{n+1, n}$. Покажем, что трансфер-матрица четырехвершинной модели $\tau_{4 \mathrm{v}}(u)$ удовлетворяет соотношению

$$
\mathrm{P} \tau_{4 \mathrm{v}}(u)=\tau_{4 \mathrm{v}}(u) \mathrm{P}=\tau_{4 \mathrm{v}}(u)
$$


Действительно, воспользовавшись явным видом матричных элементов произведения двух $L$-операторов четырехвершинной модели (здесь и ниже $L$ означает $L_{4 \mathrm{v}}$ ),

$$
L(n+1 \mid u) L(n \mid u) \equiv\left(\begin{array}{ll}
A_{n+1, n} & B_{n+1, n} \\
C_{n+1, n} & D_{n+1, n}
\end{array}\right),
$$

можно показать, что

$$
\mathscr{C}_{n+1, n} L(n+1 \mid u) L(n \mid u)=L(n+1 \mid u) L(n \mid u) \mathscr{C}_{n+1, n}=L(n+1 \mid u) L(n \mid u)
$$

и, как следствие,

$$
\mathscr{C}_{n+1, n} \tau_{4 \mathrm{v}}(u)=\tau_{4 \mathrm{v}}(u) \mathscr{C}_{n+1, n}=\tau_{4 \mathrm{v}}(u)
$$

для $n \in \mathbf{M}$ (множество $\mathbf{M}$ определено в п. 2.2). Из инвариантности матричного следа относительно циклической перестановки получаем:

$$
\mathscr{C}_{0, M} \tau_{4 \mathrm{v}}(u)=\tau_{4 \mathrm{v}}(u) \mathscr{C}_{0, M}=\tau_{4 \mathrm{v}}(u)
$$

Следовательно, соотношение (81) действительно имеет место.

Шаг II. Чтобы доказать (80), достаточно рассмотреть $\widehat{H}_{\mathrm{SA}}(67)$ при $h=0$. Воспользуемся методом, предложенным в [22] и затем примененным в [102]. Развивая этот подход для рассматриваемого случая, примем во внимание соотношение

$$
\begin{aligned}
\mathscr{C}_{n+1, n}\left[\widehat{h}_{n+1, n}, L(n+1 \mid u) L(n \mid u)\right] \mathscr{C}_{n+1, n} \\
=\mathscr{C}_{n+1, n}(Q(n+1 \mid u) L(n \mid u)-L(n+1 \mid u) Q(n \mid u)) \mathscr{C}_{n+1, n},
\end{aligned}
$$

где используется $\widehat{h}_{n+1, n}(60)$. Если (83) выполняется, то, учитывая тождество

$$
\begin{aligned}
{\left[\widehat{h}_{n+1, n}, T(u)\right]=} & L(M \mid u) \cdots L(n+2 \mid u) \\
& \times\left[\widehat{h}_{n+1, n}, L(n+1 \mid u) L(n \mid u)\right] L(n-1 \mid u) \cdots L(0 \mid u)
\end{aligned}
$$

и принимая во внимание (81), получаем, что рассматриваемый коммутатор обращается в нуль:

$$
\begin{aligned}
{\left[\widehat{H}_{\mathrm{SA}}, \tau_{4 \mathrm{v}}(u)\right] } & =-\frac{1}{2} \sum_{n=0}^{M} \mathrm{P}\left[\widehat{h}_{n+1, n}, \tau_{4 \mathrm{v}}(u)\right] \mathrm{P} \\
& =-\frac{1}{2} \sum_{n=0}^{M} \operatorname{Tr}\left(\mathrm{P}\left[\widehat{h}_{n+1, n}, T(u)\right] \mathrm{P}\right)=0 .
\end{aligned}
$$

Вернемся к (83). С одной стороны, для матричных элементов из правой части (82) получаем перестановочные соотношения

$$
\begin{aligned}
\mathscr{C}_{n+1, n}\left[\widehat{h}_{n+1, n}, A_{n+1, n}\right] \mathscr{C}_{n+1, n} & =\mathscr{C}_{n+1, n}\left(\check{q}_{n+1}-\check{q}_{n}\right) \mathscr{C}_{n+1, n}, \\
\mathscr{C}_{n+1, n}\left[\widehat{h}_{n+1, n}, B_{n+1, n}\right] \mathscr{C}_{n+1, n} & =\mathscr{C}_{n+1, n}\left(-u \sigma_{n+1}^{-}+u^{-1} \sigma_{n}^{-}\right) \mathscr{C}_{n+1, n}, \\
\mathscr{C}_{n+1, n}\left[\widehat{h}_{n+1, n}, C_{n+1, n}\right] \mathscr{C}_{n+1, n} & =\mathscr{C}_{n+1, n}\left(-u^{-1} \sigma_{n+1}^{+}+u \sigma_{n}^{+}\right) \mathscr{C}_{n+1, n}, \\
\mathscr{C}_{n+1, n}\left[\widehat{h}_{n+1, n}, D_{n+1, n}\right] \mathscr{C}_{n+1, n} & =\mathscr{C}_{n+1, n}\left(-\check{q}_{n+1}+\check{q}_{n}\right) \mathscr{C}_{n+1, n} .
\end{aligned}
$$


С другой стороны, вводя диагональную матрицу

$$
Q(n \mid u)=\left(\begin{array}{cc}
u^{-1} \mathbb{I} & 0 \\
0 & u \mathbb{I}
\end{array}\right)
$$

получаем следующее тождество для $L$-оператора (17):

$$
\begin{aligned}
Q(n+1 \mid u) L(n \mid u)-L(n+1 \mid u) Q(n \mid u) \\
\quad=\left(\begin{array}{cc}
\check{q}_{n+1}-\check{q}_{n} & -u \sigma_{n+1}^{-}+u^{-1} \sigma_{n}^{-} \\
-u^{-1} \sigma_{n+1}^{+}+u \sigma_{n}^{+} & -\check{q}_{n+1}+\check{q}_{n}
\end{array}\right) .
\end{aligned}
$$

Сопоставление (84) и (85) показывает, что соотношение (83), а следовательно, и утверждение $(80)$ действительно имеют место. Теорема доказана.

Вектор состояния (33) является собственным для трансфер-матрицы четырехвершинной модели $\tau_{4 \mathrm{v}}(u)$, а следовательно, и для гамильтониана $(67)$, если параметры $u_{i}$ удовлетворяют уравнениям Бете (70). В согласии с предложением 1, этот вектор представим в виде (76).

Далее, в работе [98] сформулировано и доказано следующее утверждение.

ПреДЛОжЕНИЕ 5. СКалярное произведение $\left\langle\Psi_{N}(\mathbf{v}) \mid \Psi_{N}(\mathbf{u})\right\rangle$ векторов состояния (76) обращается в нуль, если параметры $\mathbf{u} u \mathbf{v}$ соответствуют независимым решениям бетевских уравнений (70).

ДокАЗАтельство. Рассматриваемое скалярное произведение пропорционально определителю матрицы $\left(T_{k j}^{\circ}\right)_{1 \leqslant k, j \leqslant N}$ следующего вида:

$$
\operatorname{det} T^{\mathrm{o}} \equiv \operatorname{det}\left(h_{M-N+2}\left(u_{k}^{2} v_{j}^{-2}\right)\right)_{1 \leqslant k, j \leqslant N}=\left(\prod_{k=1}^{N} \frac{-v_{k}}{u_{k}}\right)^{N} \operatorname{det}\left(A_{k j}\right)_{1 \leqslant k, j \leqslant N},
$$

где $h_{P}(x)$ определена в $(47)$ и матричные элементы $A_{k j}$ имеют вид

$$
A_{k j}=\frac{\left(u_{k} v_{j}\right)^{M+3-N}}{u_{k}^{2}-v_{j}^{2}}\left(\prod_{\substack{1 \leqslant l \leqslant N \\ l \neq k}} u_{l}^{2}-\prod_{\substack{1 \leqslant l \leqslant N \\ l \neq j}} v_{l}^{2}\right) .
$$

При выводе правой части (86) используются уравнения Бете (70). Форм-фактор, который обобщает выражение для $\left\langle\Psi_{N}(\mathbf{v}) \mid \Psi_{N}(\mathbf{u})\right\rangle \propto \operatorname{det} T^{\circ}$, будет получен в п. 4.2 (соотношение (132)).

Определитель матрицы $\left(A_{k j}\right)_{1 \leqslant k, j \leqslant N}$ обращается в нуль при условии, что $\mathbf{u}=e^{i \boldsymbol{\theta} / 2}$ и $\mathbf{v}=e^{i \boldsymbol{\theta}^{\prime} / 2}$, где параметры $\boldsymbol{\theta}$ и $\boldsymbol{\theta}^{\prime}$ являются независимыми решениями уравнений (70). В самом деле, для матрицы $A$ существует нетривиальный собственный вектор с компонентами $\chi_{j}, 1 \leqslant j \leqslant N$, имеющий нулевое собственное значение:

$$
\sum_{j=1}^{N} A_{k j} \chi_{j}=0, \quad \chi_{j}=\left(u_{j}^{2}-v_{j}^{2}\right) \prod_{\substack{1 \leqslant l \leqslant N \\ l \neq j}} \frac{u_{j}^{2}-v_{l}^{2}}{u_{j}^{2}-u_{l}^{2}}
$$


Соотношения (87) справедливы благодаря двум тождествам:

$$
\begin{aligned}
& \sum_{k=1}^{N}\left(\frac{y_{k}-x_{k}}{y_{k}-x_{j}} \prod_{\substack{1 \leqslant l \leqslant N \\
l \neq k}} \frac{y_{k}-x_{l}}{y_{k}-y_{l}}\right)=1, \\
& \sum_{k=1}^{N}\left(\frac{y_{k}-x_{k}}{y_{k}} \prod_{\substack{1 \leqslant l \leqslant N \\
l \neq k}} \frac{y_{k}-x_{l}}{y_{k}-y_{l}}\right)=1-\prod_{l=1}^{N} \frac{x_{l}}{y_{l}} .
\end{aligned}
$$

Таким образом, $\left\langle\Psi_{N}\left(e^{i \boldsymbol{\theta}^{\prime} / 2}\right) \mid \Psi_{N}\left(e^{i \boldsymbol{\theta} / 2}\right)\right\rangle$ обращается в нуль, т. е. векторы состояния ортогональны, если множества параметров $\boldsymbol{\theta}$ и $\boldsymbol{\theta}^{\prime}$ соответствуют независимым решениям уравнений (70). Предложение доказано.

Для общего случая $X X Z$-модели в работе [77] представлено доказательство ортогональности собственных состояний трансфер-матрицы, отвечающих двум независимым наборам бетевских решений. Доказательство в [98] основано на представлении (76) для вектора состояния при бесконечной анизотропии.

Итак, эффективный гамильтониан $\widehat{H}_{\mathrm{SA}}(67)$ перестановочен с трансфер-матрицей $\tau_{4 \mathrm{v}}(u)$, которая возникает из трансфер-матрицы шестивершинной модели в пределе бесконечной анизотропии. Кроме того, полнота набора состояний для $\widehat{H}_{\mathrm{SA}}$ была доказана в [23], [101]. Следовательно, гамильтониан $\widehat{H}_{\mathrm{SA}}(67)$ и изинговский гамильтониан $\widehat{H}_{\mathrm{IZ}}(66)$ обладают общей полной ортогональной системой собственных функций. Поэтому как гамильтониан $\widehat{H}_{\mathrm{SA}}(67)$, так и векторы состояния (76) являются приемлемыми для исследования изинговского предела $X X Z$-модели.

\section{4. Корреляционные функции $X X Z$-магнетика при нулевой и бесконечной анизотропии}

\section{1. Форм-факторы и корреляционные функции при нулевой ани- зотропии.}

4.1.1. Определения. Основной целью (как при $\Delta=0$, так и при $\Delta \rightarrow-\infty$ ) будет вычисление и исследование температурных корреляционных функций, которые назовем выживанием ферромагнитной струны и выживанием доменной стенки.

Выживание ферромагнитной струны связано с оператором $\bar{\Pi}_{n}$, обеспечивающим отсутствие частиц на $n$ последовательных узлах цепочки:

$$
\mathscr{T}\left(\boldsymbol{\theta}_{N}^{\mathrm{g}}, n, \beta\right) \equiv \frac{\left\langle\Psi_{N}\left(\boldsymbol{\theta}^{\mathrm{g}}\right)\left|\bar{\Pi}_{n} e^{-\beta \mathscr{H}} \bar{\Pi}_{n}\right| \Psi_{N}\left(\boldsymbol{\theta}^{\mathrm{g}}\right)\right\rangle}{\left\langle\Psi_{N}\left(\boldsymbol{\theta}^{\mathrm{g}}\right)\left|e^{-\beta \mathscr{H}}\right| \Psi_{N}\left(\boldsymbol{\theta}^{\mathrm{g}}\right)\right\rangle}, \quad \bar{\Pi}_{n} \equiv \prod_{j=0}^{n-1} \check{q}_{j},
$$

где $\beta$ - обратная температура, $\check{q}_{j}$ определено в $(68)$ и $\boldsymbol{\theta}_{N}^{\mathrm{g}}=\left(\theta_{1}^{\mathrm{g}}, \theta_{2}^{\mathrm{g}}, \ldots, \theta_{N}^{\mathrm{g}}\right)$ отвечает $N$-частичному основному состоянию. Здесь и далее до конца раздела 4 будем использовать $\mathscr{H}$ для обозначения гамильтониана $\widehat{H}_{X X}$ в случае нулевой анизотропии. Кроме того, будем упрощать обозначения, используя $\left|\Psi_{N}\left(\boldsymbol{\theta}^{\mathrm{g}}\right)\right\rangle$ вместо $\left|\Psi_{N}\left(e^{i \boldsymbol{\theta}_{N}^{\mathrm{g}} / 2}\right)\right\rangle$. Использование $\boldsymbol{\theta}$ вместо $\boldsymbol{\theta}_{N}$ также допускается, когда нет двусмысленности. 
Выживание доменной стенки связано с оператором $\overline{\mathrm{F}}_{n}$, порождающим $n$ последовательных спинов "вниз":

$$
\mathscr{F}\left(\boldsymbol{\theta}_{N-n}^{\mathrm{g}}, n, \beta\right) \equiv \frac{\left\langle\Psi_{N-n}\left(\boldsymbol{\theta}^{\mathrm{g}}\right)\left|\overline{\mathrm{F}}_{n} e^{-\beta \mathscr{H}} \overline{\mathrm{F}}_{n}\right| \Psi_{N-n}\left(\boldsymbol{\theta}^{\mathrm{g}}\right)\right\rangle}{\left\langle\Psi_{N-n}\left(\boldsymbol{\theta}^{\mathrm{g}}\right)\left|e^{-\beta \mathscr{H}}\right| \Psi_{N-n}\left(\boldsymbol{\theta}^{\mathrm{g}}\right)\right\rangle}, \quad \overline{\mathrm{F}}_{n} \equiv \prod_{j=0}^{n-1} \sigma_{j}^{-},
$$

где $\boldsymbol{\theta}_{N-n}^{\mathrm{g}}=\left(\theta_{1}^{\mathrm{g}}, \theta_{2}^{\mathrm{g}}, \ldots, \theta_{N-n}^{\mathrm{g}}\right)$ соответствует (справа на это же указывает нижний значок в обозначении вектора состояния) $(N-n)$-частичному решению уравнений Бете (62) для основного состояния

$$
\theta_{j}^{\mathrm{g}}=\frac{2 \pi}{M+1}\left(\frac{N-n+1}{2}-j\right), \quad 1 \leqslant j \leqslant N-n .
$$

При этом мы полагаем $\bar{\Pi}_{0}$ и $\overline{\mathrm{F}}_{0}$ равными тождественному оператору $\mathbf{I}$, т. е. $\mathscr{T}\left(\boldsymbol{\theta}_{N}^{\mathrm{g}}, 0, \beta\right)=1$ и $\mathscr{F}\left(\boldsymbol{\theta}_{N-n}^{\mathrm{g}}, 0, \beta\right)=1$.

4.1.2. Форм-факторы. В случае, описываемом ХХ-гамильтонианом (60), использование формул $(75),(77),(78)$ и (79) позволяет получить форм-фактор

$$
\left\langle\Psi_{N}(\mathbf{v})\left|\bar{\Pi}_{n}\right| \Psi_{N}(\mathbf{u})\right\rangle=\mathscr{P}_{\mathscr{M} / n}\left(\mathbf{v}^{-2}, \mathbf{u}^{2}\right)=\left(\prod_{l=1}^{N} \frac{u_{l}^{2 n}}{v_{l}^{2 n}}\right) \frac{\operatorname{det}\left(T_{k j}\right)_{1 \leqslant k, j \leqslant N}}{\mathscr{V}_{N}\left(\mathbf{u}^{2}\right) \mathscr{V}_{N}\left(\mathbf{v}^{-2}\right)},
$$

где матричные элементы имеют вид

$$
T_{k j}=\frac{1-\left(u_{k}^{2} / v_{j}^{2}\right)^{M+1-n}}{1-u_{k}^{2} / v_{j}^{2}} .
$$

Соотношение (91) превращается при $n=0$ в представление для скалярного произведения $\left\langle\Psi_{N}(\mathbf{v}) \mid \Psi_{N}(\mathbf{u})\right\rangle$, которое становится при $\mathbf{v}=\mathbf{u}$ "квадратом длины" $\mathscr{N}^{2}(\mathbf{u}) \equiv\left\langle\Psi_{N}(\mathbf{u}) \mid \Psi_{N}(\mathbf{u})\right\rangle$ состояний (75). На решениях $\mathbf{u}^{2}=\mathbf{v}^{2}=e^{i \boldsymbol{\theta}}$ (63) имеем квадрат нормы:

$$
\begin{aligned}
\mathscr{N}^{2}(\boldsymbol{\theta}) \equiv \mathscr{N}^{2}\left(e^{i \boldsymbol{\theta} / 2}\right) & =\frac{(M+1)^{N}}{\left|\mathscr{V}_{N}\left(e^{i \boldsymbol{\theta}}\right)\right|^{2}} \\
& =\frac{(M+1)^{N}}{\prod_{1 \leqslant m<l \leqslant N} 2\left(1-\cos (2 \pi /(M+1))\left(I_{l}-I_{m}\right)\right)} .
\end{aligned}
$$

Так как бетевские векторы состояния $\left|\Psi_{N}\left(e^{i \boldsymbol{\theta} / 2}\right)\right\rangle$ образуют полный и ортогональный набор, то разложение тождественного оператора принимает следующий вид [70], [71]:

$$
\mathbf{I}=\sum_{\{\boldsymbol{\theta}\}} \mathscr{N}^{-2}(\boldsymbol{\theta})\left|\Psi_{N}\left(e^{i \boldsymbol{\theta} / 2}\right)\right\rangle\left\langle\Psi_{N}\left(e^{i \boldsymbol{\theta} / 2}\right)\right|,
$$

где суммирование идет по всем наборам независимых решений (63).

Рассмотрим отношение

$$
\mathscr{T}(\mathbf{v}, \mathbf{u}, n) \equiv \frac{\left\langle\Psi_{N}(\mathbf{v})\left|\bar{\Pi}_{n}\right| \Psi_{N}(\mathbf{u})\right\rangle}{\mathscr{N}(\mathbf{v}) \mathscr{N}(\mathbf{u})}
$$


где $\bar{\Pi}_{n}$ - проектор (88). На бетевских решениях (63) нормированное среднее (95) принимает вид

$$
\mathscr{T}\left(e^{i \boldsymbol{\theta} / 2}, e^{i \boldsymbol{\theta} / 2}, n\right)=\operatorname{det}\left(\delta_{l m}-K_{n}\left(\theta_{l}, \theta_{m}\right)\right)_{1 \leqslant l, m \leqslant N},
$$

где матричные элементы $K_{n}\left(\theta_{k}, \theta_{j}\right)$ равны

$$
K_{n}\left(\theta_{k}, \theta_{j}\right) \equiv \frac{e^{i(n-1)\left(\theta_{k}-\theta_{j}\right) / 2}}{M+1} \frac{\sin \left(n\left(\theta_{k}-\theta_{j}\right) / 2\right)}{\sin \left(\left(\theta_{k}-\theta_{j}\right) / 2\right)} .
$$

Соотношение (96) известно как вероятность образования пустоты, т. е. вероятность образования цепочки $n$ последовательных спинов “вверх" [73], [76], [77], [103].

Другой коррелятор связан с оператором рождения доменной стенки $\overline{\mathrm{F}}_{n}(89)$ :

$$
\mathscr{F}\left(\mathbf{v}_{N}, \mathbf{u}_{N-n}, n\right) \equiv \frac{\left\langle\Psi_{N}\left(\mathbf{v}_{N}\right)\left|\overline{\mathrm{F}}_{n}\right| \Psi_{N-n}\left(\mathbf{u}_{N-n}\right)\right\rangle}{\mathscr{N}\left(\mathbf{v}_{N}\right) \mathscr{N}\left(\mathbf{u}_{N-n}\right)},
$$

где $\mathbf{v}_{N} \equiv\left(v_{1}, v_{2}, \ldots, v_{N}\right), \mathbf{u}_{N-n} \equiv\left(u_{1}, u_{2}, \ldots, u_{N-n}\right)$. Для его вычисления введем вспомогательный оператор $\mathrm{D}^{n}(\mathbf{u})$, действующий на функцию, которая зависит от набора аргументов $\mathbf{u}$ и обозначается через $\langle\cdots\rangle_{\mathbf{u}}$. В последующем $\langle\cdots\rangle_{\mathbf{u}}$ приобретет смысл коррелятора. Действие $\mathrm{D}^{n}(\mathbf{u})$ определяется следующим правилом:

$$
\mathrm{D}^{n}(\mathbf{u})\langle\cdot\rangle_{\mathbf{u}} \equiv \mathrm{D}_{u_{N-n+1}, u_{N-n+2}, \ldots, u_{N}}\left(\frac{\mathscr{V}_{N}\left(\mathbf{u}_{N}^{2}\right)}{\mathscr{V}_{N-n}\left(\mathbf{u}_{N-n}^{2}\right)} \times\langle\cdot\rangle_{\mathbf{u}}\right) .
$$

Здесь $\mathrm{D}_{u_{N-n+1}, u_{N-n+2}, \ldots, u_{N}}$ означает последовательное $n$-кратное применение операторов дифференцирования $\mathrm{D}_{u_{N-j}}^{j}$ :

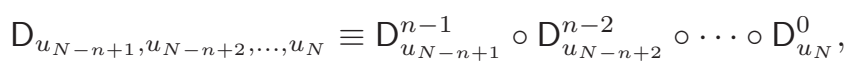

где

$$
\mathrm{D}_{u_{N-j}}^{j} \equiv \lim _{u_{N-j}^{2} \rightarrow 0} \frac{1}{j !} \frac{d^{j}}{d\left(u_{N-j}^{2}\right)^{j}}, \quad 0 \leqslant j \leqslant n-1 .
$$

Определение (98) означает, что $\langle\cdot\rangle_{\mathbf{u}}$ сначала умножается на отношение определителей Вандермонда, а результат дифференцируется $n$ раз. С учетом введенных определений имеет место следующая теорема.

Теорема 2. Действие оператора $\mathrm{D}^{n}(\mathbf{u})$, заданного соотношениями (98), (99), (100), на скалярное произведение $\left\langle\Psi_{N}(\mathbf{v}) \mid \Psi_{N}(\mathbf{u})\right\rangle$ дает форм-фбактор оператора рождения доменной стенки $\overline{\mathrm{F}}_{n}(89)$ :

$$
\left\langle\Psi_{N}(\mathbf{v})\left|\overline{\mathrm{F}}_{n}\right| \Psi_{N-n}(\mathbf{u})\right\rangle=\mathrm{D}^{n}(\mathbf{u})\left\langle\Psi_{N}(\mathbf{v}) \mid \Psi_{N}(\mathbf{u})\right\rangle,
$$

где

$$
\left|\Psi_{N-n}(\mathbf{u})\right\rangle \equiv\left|\Psi_{N-n}\left(\mathbf{u}_{N-n}\right)\right\rangle, \quad\left\langle\Psi_{N}(\mathbf{v})\right| \equiv\left\langle\Psi_{N}\left(\mathbf{v}_{N}\right)\right| .
$$

Значения $u_{N-n+1}, \ldots, u_{N}$, входящие в $\mathbf{u}=\mathbf{u}_{N}=\left(\mathbf{u}_{N-n}, u_{N-n+1}, \ldots, u_{N}\right)$ в правой части (101), используются формально, чтобы выразить $\left\langle\Psi_{N}(\mathbf{v}) \mid \Psi_{N}(\mathbf{u})\right\rangle$. 
ДокАЗАТЕЛЬство. Определения векторов состояния (75) и (77) приводят к форм-фактору оператора $\bar{F}_{n}$ в виде суммы [80]:

$$
\left\langle\Psi_{N}(\mathbf{v})\left|\overline{\mathrm{F}}_{n}\right| \Psi_{N-n}(\mathbf{u})\right\rangle=\left(\prod_{l=1}^{N-n} u_{l}^{2 n}\right) \sum_{\boldsymbol{\lambda} \subseteq\left\{\mathscr{M}^{N-n}\right\}} S_{\widehat{\boldsymbol{\lambda}}}\left(\mathbf{v}_{N}^{-2}\right) S_{\boldsymbol{\lambda}}\left(\mathbf{u}_{N-n}^{2}\right),
$$

где сумма имеет тот же смысл, что в $(75)$, и для $\boldsymbol{\lambda}$ имеем $l(\boldsymbol{\lambda})=N-n$. Разбиение $\hat{\boldsymbol{\lambda}}_{\text {в }}(102)$ имеет длину $l(\hat{\boldsymbol{\lambda}})=N$, так как содержит дополнительные нули: $\widehat{\lambda}_{p}=\lambda_{p}$ при $1 \leqslant p \leqslant N-n$ и $\widehat{\lambda}_{N-n+1}=\widehat{\lambda}_{N-n+2}=\cdots=\widehat{\lambda}_{N}=0$. Соответствующие строгие разбиения имеют вид $\widehat{\boldsymbol{\mu}}=\widehat{\boldsymbol{\lambda}}+\boldsymbol{\delta}_{N}$ и $\boldsymbol{\mu}=\boldsymbol{\lambda}+\boldsymbol{\delta}_{N-n}$. Для вывода (102) действуем оператором $\bar{F}_{n}$ на состояние $\left|\Psi_{N-n}(\mathbf{u})\right\rangle$ вида (75), но с областью суммирования $\boldsymbol{\lambda} \subseteq\left\{(\mathscr{M}+n)^{N-n}\right\}$. Действие $\overline{\mathrm{F}}_{n}$ нетривиально только на тех векторах, входящих в $\left|\Psi_{N-n}(\mathbf{u})\right\rangle$, которые не имеют спинов "вниз" на первых $n$ узлах:

$$
\overline{\mathrm{F}}_{n}\left|\Psi_{N-n}(\mathbf{u})\right\rangle=\left(\prod_{l=1}^{N-n} u_{l}^{2 n}\right) \sum_{\boldsymbol{\lambda} \subseteq\left\{\mathscr{M}^{N-n}\right\}} S_{\boldsymbol{\lambda}}\left(\mathbf{u}_{N-n}^{2}\right)\left(\prod_{l=0}^{n-1} \sigma_{l}^{-}\right)\left(\prod_{k=1}^{N-n} \sigma_{\mu_{k}}^{-}\right)|\Uparrow\rangle .
$$

Пользуясь ортогональностью состояний (75) и (77), переходим от (103) к (102). Вычисляя правую часть (101) дифференцированием форм-фактора (91), взятого при $n=0$ в виде ряда, также получаем правую часть (102). Теорема доказана.

Теорема 2 позволяет получить два правила суммирования произведений функций Шура, которые существенны для получения комбинаторных соотношений. Таким образом, возникает следующая теорема.

ТЕОРема 3. Для сумм произведений функиий Шура имеют место следующие соотношения:

$$
\begin{aligned}
\sum_{\boldsymbol{\lambda} \subseteq\left\{\mathscr{M}^{N-n}\right\}} S_{\widehat{\boldsymbol{\lambda}}}\left(\mathbf{v}_{N}^{-2}\right) S_{\boldsymbol{\lambda}}\left(\mathbf{u}_{N-n}^{2}\right) & =\left(\prod_{l=1}^{N-n} u_{l}^{-2 n}\right) \frac{\operatorname{det}\left(\bar{T}_{k j}\right)_{1 \leqslant k, j \leqslant N}}{\mathscr{V}_{N-n}\left(\mathbf{u}_{N-n}^{2}\right) \mathscr{V}_{N}\left(\mathbf{v}_{N}^{-2}\right)}, \\
\sum_{\boldsymbol{\lambda} \subseteq\left\{\mathscr{M}^{N-n}\right\}} S_{\boldsymbol{\lambda}}\left(\mathbf{v}_{N-n}^{-2}\right) S_{\widehat{\boldsymbol{\lambda}}}\left(\mathbf{u}_{N}^{2}\right) & =\left(\prod_{l=1}^{N-n} v_{l}^{2 n}\right) \frac{\operatorname{det}\left(\widetilde{T}_{k j}\right)_{1 \leqslant k, j \leqslant N}}{\mathscr{V}_{N-n}\left(\mathbf{v}_{N-n}^{-2}\right) \mathscr{V}_{N}\left(\mathbf{u}_{N}^{2}\right)}
\end{aligned}
$$

где $\left(\bar{T}_{k j}\right)_{1 \leqslant k, j \leqslant N} u\left(\widetilde{T}_{k j}\right)_{1 \leqslant k, j \leqslant N}$ - матричные элементъ:

$$
\begin{array}{lll}
\bar{T}_{k j}=T_{k j}^{\circ}, & 1 \leqslant k \leqslant N-n, & 1 \leqslant j \leqslant N, \\
\bar{T}_{k j}=v_{j}^{-2(N-k)}, & N-n+1 \leqslant k \leqslant N, & 1 \leqslant j \leqslant N,
\end{array}
$$

$u$

$$
\begin{array}{ll}
\widetilde{T}_{k j}=T_{k j}^{\mathrm{o}}, & 1 \leqslant k \leqslant N, \quad 1 \leqslant j \leqslant N-n, \\
\widetilde{T}_{k j}=u_{j}^{2(N-k)}, & 1 \leqslant k \leqslant N, \quad N-n+1 \leqslant j \leqslant N .
\end{array}
$$

Матричнъе элементы $T_{k j}^{o}$ совпадают с $T_{k j}(92)$, взятыми при $n=0$. 
ДокАЗАТЕЛЬСтво. Вычислим правую часть соотношения (101), используя представление для скалярного произведения в виде определителя (в (91) при $n=0$ заменяем $T_{k j}$ на $\left.T_{k j}^{\mathrm{o}}\right)$ :

$$
\left\langle\Psi_{N}(\mathbf{v})\left|\overline{\mathrm{F}}_{n}\right| \Psi_{N-n}(\mathbf{u})\right\rangle=\mathrm{D}^{n}(\mathbf{u})\left(\frac{\operatorname{det}\left(T_{k j}^{\mathrm{o}}\right)_{1 \leqslant k, j \leqslant N}}{\mathscr{V}_{N}\left(\mathbf{u}^{2}\right) \mathscr{V}_{N}\left(\mathbf{v}^{-2}\right)}\right) .
$$

Далее, учитывая (92), получаем из (107) равенство

$$
\left\langle\Psi_{N}(\mathbf{v})\left|\overline{\mathrm{F}}_{n}\right| \Psi_{N-n}(\mathbf{u})\right\rangle=\frac{\operatorname{det}\left(\bar{T}_{k j}\right)_{1 \leqslant k, j \leqslant N}}{\mathscr{V}_{N-n}\left(\mathbf{u}^{2}\right) \mathscr{V}_{N}\left(\mathbf{v}^{-2}\right)}
$$

где элементы матрицы $\bar{T}$ определены в (106). Так как правые части (102) и (108) совпадают, то соотношение для функций Шура (104) (которое, в принципе, того же типа, что и (78)) имеет место. Действуя аналогично, можно установить соотношение (105). Теорема доказана.

4.1.3. Производящие функции случайных блужданий недружественных пешеходов. Проблеме перечисления случайных блужданий по одномерной решетке недружественных пешеходов, которые при встрече в любом из узлов решетки уничтожают друг друга, посвящена обширная литература [44]-[50], [58], [59], [104]-[106]. Как показано в [58], [59], переходы между состояниями спинов "вверх" и "вниз" на узлах спиновой цепочки можно интерпретировать как случайные перемещения пешеходов в модели блужданий со случайнъми поворотами. Блуждания со случайными поворотами характеризуются тем, что в каждый момент времени только один из пешеходов, выбранный случайно, делает один шаг влево или вправо, а остальные остаются на своих местах. При этом из спиновых средних над ферромагнитным состоянием $X X 0$-магнетика в нулевом магнитном поле можно построить производящие функции случайных блужданий недружественных пешеходов [58], [59]. Парная корреляционная функция спинов, вычисленная по всем собственным состояниям магнетика, интерпретируется в [60] как производящая функция блужданий одиночного пешехода в среде с переменным числом недружественных соседей.

В этом разделе мы рассмотрим, следуя [58]-[60], производящие функции случайных блужданий недружественных пешеходов. Гамильтониан $\mathscr{H}$ (60) (т. е. гамильтониан (54) при $\Delta=h=0$ ) используется в [60] с нумерацией узлов, изменяющейся от 1 до $M(M=0(\bmod 2))$. Кроме того, введена матрица перескоков $\Delta_{n m}^{(s)}$ :

$$
\Delta_{n m}^{(s)} \equiv \frac{1}{2}\left(\delta_{|n-m|, 1}+s \delta_{|n-m|, M-1}\right)
$$

где параметр $s$ связан с выбором периодических $(s=+1)$ или антипериодических $(s=-1)$ граничных условий. С учетом (109) гамильтониан периодической $X X$-модели принимает следующий вид: $-\sum_{n, m=1}^{M} \Delta_{n m}^{(+)} \sigma_{n}^{+} \sigma_{m}^{-}$. Далее рассматри-

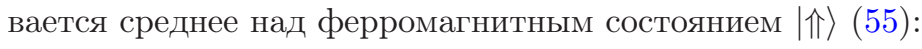

$$
F_{j ; l}(\beta) \equiv\left\langle\Uparrow\left|\sigma_{j}^{+} e^{-\beta \mathscr{H}} \sigma_{l}^{-}\right| \Uparrow\right\rangle,
$$


где $\beta \in \mathbb{C}$ можно рассматривать как "эволюционный" параметр. Спины "вверх" (или “вниз") соответствуют пустым (или занятым) узлам цепочки. Дифференцируя $F_{j ; l}(\beta)(110)$ и раскрывая коммутатор $\left[\mathscr{H}, \sigma_{j}^{+}\right]$, мы получаем разностно-дифференциальное уравнение

$$
\frac{d}{d \beta} F_{j ; l}(\beta)=\frac{1}{2}\left(F_{j+1 ; l}(\beta)+F_{j-1 ; l}(\beta)\right) .
$$

Решение уравнения (111) определяется требованием периодичности по решеточному аргументу, а также "начальным" условием при $\beta=0$ и принимает вид

$$
F_{k ; l}(\beta) \equiv \frac{1}{M+1} \sum_{s=0}^{M} e^{\beta \cos \phi_{s}} e^{i \phi_{s}(k-l)}, \quad \phi_{s}=\frac{2 \pi}{M+1}\left(s-\frac{M}{2}\right) .
$$

Среднее $F_{j ; l}(\beta)$ (110) можно рассматривать как производящую функцию траекторий со случайными поворотами, начинающихся в $l$-м узле и оканчивающихся в $j$-м узле цепочки. Действительно, введем обозначение $\mathscr{D}_{\ell}^{K}$ для оператора $K$-кратного дифференцирования по $\ell$ в точке $\ell=0$. Применение $\mathscr{D}_{\beta}^{K}$ к (110) дает

$$
\mathscr{D}_{\beta}^{K}\left[F_{j ; l}(\beta)\right]=\left\langle\Uparrow\left|\sigma_{j}^{+}(-\mathscr{H})^{K} \sigma_{l}^{-}\right| \Uparrow\right\rangle=\sum_{n_{1}, \ldots, n_{K-1}} \Delta_{j n_{K-1}}^{(+)} \cdots \Delta_{n_{2} n_{1}}^{(+)} \Delta_{n_{1} l}^{(+)} .
$$

Правая часть (113) совпадает с элементом $j$-й строки и $l$-го столбца в произведении $K$ матриц перескоков (109). Каждая матрица соответствует переходу между двумя ближайшими узлами. Будучи умноженной на $2^{K}$ (что связано с принятой нормировкой матрицы (109)), правая часть (113) дает число траекторий из $K$ звеньев между $l$-м и $j$-м узлами. Обозначим это число $\left|P_{K}(l \rightarrow j)\right|$.

Рассмотрим бесконечную цепочку $(M \rightarrow \infty)$. В этом случае решением уравнения (111), удовлетворяющим условию $F_{j ; l}(0)=\delta_{j l}$, оказывается модифицированная функция Бесселя $I_{j-l}(\beta)[58]$ :

$$
F_{j ; l}(\beta)=I_{j-l}(\beta)=\frac{1}{2 \pi} \int_{-\pi}^{\pi} e^{\beta \cos \theta} e^{i(j-l) \theta} d \theta .
$$

Пусть $K$ удовлетворяет $K \geqslant|l-j|$ и $K+|j-l|=0(\bmod 2)$. Тогда дифференцирование формулы (114)) приводит к биномиальной формуле $\left|P_{K}(l \rightarrow j)\right|=C_{K}^{L}$ для числа всех решеточных путей “длины” $K$ между двумя узлами на бесконечной оси:

$$
\left|P_{K}(l \rightarrow j)\right| \equiv \mathscr{D}_{\beta / 2}^{K}\left[F_{j ; l}(\beta)\right]=\frac{(m+2 L) !}{L !(m+L) !},
$$

где $L \equiv(K-m) / 2$ означает половину числа поворотов.

Перейдем к многоточечной корреляционной функции, определяемой соотношением

$$
F_{\boldsymbol{\mu}^{\mathrm{L}} ; \boldsymbol{\mu}^{\mathrm{R}}}(\beta) \equiv\left\langle\Uparrow\left|\left(\prod_{l=1}^{N} \sigma_{\mu_{l}^{\mathrm{L}}}^{+}\right) e^{-\beta \mathscr{H}}\left(\prod_{p=1}^{N} \sigma_{\mu_{p}^{\mathrm{R}}}^{-}\right)\right| \Uparrow\right\rangle,
$$

где начальное и конечное положения пешеходов на узлах задаются частями строгих разбиений $\boldsymbol{\mu}^{\mathrm{L}} \equiv\left(\mu_{1}^{\mathrm{L}}, \mu_{2}^{\mathrm{L}}, \ldots, \mu_{N}^{\mathrm{L}}\right)$ и $\boldsymbol{\mu}^{\mathrm{R}} \equiv\left(\mu_{1}^{\mathrm{R}}, \mu_{2}^{\mathrm{R}}, \ldots, \mu_{N}^{\mathrm{R}}\right)$. Как показано в [58] и [59], 2N-точечная корреляционная функция над ферромагнитным 
состоянием (115) связана с перечислением допустимых траекторий $N$ недружественных пешеходов, перемещающихся по узлам одномерной решетки в модели блужданий со случайными поворотами.

Коррелятор (115) удовлетворяет дифференциально-разностному уравнению, выведенному в [59], которое для случая периодичности по решеточному аргументу и при "начальном" условии $F_{\boldsymbol{\mu}^{\mathrm{L} ; \mu^{\mathrm{R}}}}(0)=\prod_{k=1}^{N} \delta_{\mu_{k}^{\mathrm{L}}, \mu_{k}^{\mathrm{R}}}$ приводит к решению
в виде определителя:

$$
F_{\boldsymbol{\mu}^{\mathrm{L}} ; \boldsymbol{\mu}^{\mathrm{R}}}(\beta)=\operatorname{det}\left(F_{\mu_{k}^{\mathrm{L}} ; \mu_{l}^{\mathrm{R}}}(\beta)\right)_{1 \leqslant k, l \leqslant N}
$$

Матричные элементы в (116) являются амплитудами перехода (110) и принимают вид (112). Соответствующее решение задается при $N=1$ суммой (112), которая в пределе $M \rightarrow \infty$ приводит к функции Бесселя (114).

Пусть $\left|P_{K}\left(\boldsymbol{\mu}^{\mathrm{L}} \rightarrow \boldsymbol{\mu}^{\mathrm{R}}\right)\right|$ обозначает количество траекторий, состоящих из $K$ звеньев, которые описаны $N$ недружественными пешеходами в модели со случайными поворотами. Числа $\left|P_{K}\left(\boldsymbol{\mu}^{\mathrm{L}} \rightarrow \boldsymbol{\mu}^{\mathrm{R}}\right)\right|$ возникают в результате применения $\mathscr{D}_{\beta / 2}^{K}$ к коррелятору (115) (см. [59], [60]). Условие непересечения траекторий пешеходов проявляется в обращении в нуль выражения (115) для любой пары совпадающих индексов $\mu_{k}^{\mathrm{R}}$ или $\mu_{p}^{\mathrm{L}}$ (на рис. 8 показана типичная конфигурация путей для трех пешеходов). Число траекторий $N$ недружественных пешеходов $\left|P_{K}\left(\boldsymbol{\mu}^{\mathrm{L}} \rightarrow \boldsymbol{\mu}^{\mathrm{R}}\right)\right|$ может быть выражено в терминах случайных блужданий той же “длины” $K$ для одного пешехода, перемещающегося по узлам $N$-мерной бесконечной гиперкубической решетки [60].

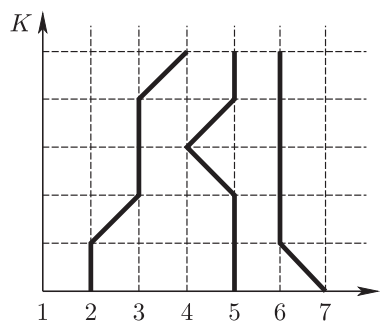

Рис. 8. Конфигурация путей трех недружественных пешеходов $\left(\boldsymbol{\mu}^{\mathrm{L}}=\right.$ $\left.(2,5,7), \boldsymbol{\mu}^{\mathrm{R}}=(4,5,6)\right)$ в модели блужданий со случайными поворотами

Подстановка (112) в (116) позволяет выразить амплитуду (115) через функции Шура и определители Вандермонда, введенные в (35) (см. [58]):

$$
F_{\boldsymbol{\mu}^{\mathrm{L}} ; \boldsymbol{\mu}^{\mathrm{R}}}(\beta)=\frac{1}{(M+1)^{N}} \sum_{\left\{\boldsymbol{\phi}_{N}\right\}} e^{-\beta E_{N}\left(\phi_{N}\right)}\left|\mathscr{V}_{N}\left(e^{i \boldsymbol{\phi}_{N}}\right)\right|^{2} S_{\boldsymbol{\lambda}^{\mathrm{L}}}\left(e^{i \phi_{N}}\right) S_{\boldsymbol{\lambda}^{\mathrm{R}}}\left(e^{-i \phi_{N}}\right) .
$$

Суммирование в (117) идет по $N$-наборам $\phi_{N} \equiv\left(\phi_{k_{1}}, \phi_{k_{2}}, \ldots, \phi_{k_{N}}\right)$, где целые числа $k_{i}, 1 \leqslant i \leqslant N$, удовлетворяют условиям $M \geqslant k_{1}>k_{2}>\cdots>k_{N} \geqslant 0$. Энергия $E_{N}\left(\phi_{N}\right)$ определена в (64). В представлении (117) положим $\boldsymbol{\mu}^{\mathrm{R}}=\boldsymbol{\delta}_{N}$, 
где $\boldsymbol{\delta}_{N}=(N-1, N-2, \ldots, 1,0)$. Тогда $S_{\boldsymbol{\lambda}^{\mathrm{R}}}\left(e^{-i \phi_{N}}\right)=1$, и при $M \gg 1$ получаем

$$
\begin{gathered}
F_{\boldsymbol{\mu} ; \boldsymbol{\delta}_{N}}(\beta)=\frac{e^{\beta N}}{N !} \int_{-\pi}^{\pi} \int_{-\pi}^{\pi} \cdots \int_{-\pi}^{\pi} \exp \left\{\beta \sum_{l=1}^{N}\left(\cos \phi_{l}-1\right)\right\} S_{\boldsymbol{\lambda}}\left(e^{i \phi}\right) \\
\times \prod_{1 \leqslant k<l \leqslant N}\left|e^{i \phi_{k}}-e^{i \phi_{l}}\right|^{2} \frac{d \phi_{1} d \phi_{2} \cdots d \phi_{N}}{(2 \pi)^{N}},
\end{gathered}
$$

где $e^{i \phi} \equiv\left(e^{i \phi_{1}}, e^{i \phi_{2}}, \ldots, e^{i \phi_{N}}\right), \boldsymbol{\mu} \equiv \boldsymbol{\mu}^{\mathrm{L}}$ и $\boldsymbol{\lambda} \equiv \boldsymbol{\lambda}^{\mathrm{L}}$. Ведущая асимптотика (118) при $\beta \rightarrow \infty$ имеет вид

$$
\begin{gathered}
F_{\boldsymbol{\mu} ; \boldsymbol{\delta}_{N}}(\beta) \simeq S_{\boldsymbol{\lambda}}(\mathbf{1}) \frac{e^{\beta N} \mathscr{I}_{N}}{\beta^{N^{2} / 2}}, \\
\mathscr{I}_{N} \equiv \frac{1}{N !} \int_{-\infty}^{\infty} \int_{-\infty}^{\infty} \cdots \int_{-\infty}^{\infty} \exp \left\{-\frac{1}{2} \sum_{l=1}^{N} x_{l}^{2}\right\} \prod_{1 \leqslant k<l \leqslant N}\left|x_{k}-x_{l}\right|^{2} \frac{d x_{1} d x_{2} \cdots d x_{N}}{(2 \pi)^{N}},
\end{gathered}
$$

где $S_{\boldsymbol{\lambda}}(\mathbf{1}) \equiv S_{\boldsymbol{\lambda}}(1,1, \ldots, 1)$. Интеграл $\mathscr{I}_{N}(120)$ есть специальная форма интеграла Мехты, связанного со статистической суммой гауссова унитарного ансамбля [81], [107]. Значение $S_{\boldsymbol{\lambda}}(\mathbf{1})$ совпадает с размерностью неприводимого представления унитарной группы, а кроме того, может интерпретироваться как число наборов самоизбегающих решеточных путей (см. (А.22) в приложении А).

С учетом (75) оказывается, что нормированное среднее

$$
\left\langle\sigma_{m+l}^{+} e^{-\beta \mathscr{H}} \sigma_{l}^{-}\right\rangle_{N} \equiv \frac{\left\langle\Psi_{N}\left|\sigma_{m+l}^{+} e^{-\beta \mathscr{H}} \sigma_{l}^{-}\right| \Psi_{N}\right\rangle}{\left\langle\Psi_{N} \mid \Psi_{N}\right\rangle}
$$

(корреляционная функция спинов, вычисленная по всем собственным состояниям) может быть представлено как билинейная комбинация $(N+1)$-точечных производящих функций типа (115) и, таким образом, связано с блужданиями $N+1$ пешехода. Начальное и конечное положения одного из них зафиксированы на $l$-м и $(m+l)$-м узлах, в то время как для остальных выбор положений случаен.

4.1.4. Корреляционные функции выживания ферромагнитной струны и доменной стенки. Перейдем к вычислению корреляционных функций типа выживания ферромагнитной струны и доменной стенки.

Используя определения (75) и (77), получаем следующую амплитуду перехода [97]:

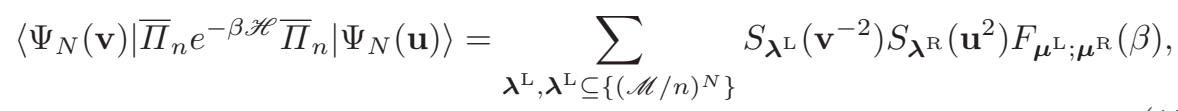


где $\mathbf{u}$ и $\mathbf{v}$ означают произвольную параметризацию и $F_{\boldsymbol{\mu}^{\mathrm{L}} ; \boldsymbol{\mu}^{\mathrm{R}}}(\beta)$ задается соотношением (115). Область суммирования по $\lambda^{\mathrm{L}}$ и $\lambda^{\mathrm{R}}$ определена в (78), и $\boldsymbol{\mu}^{\mathrm{L}, \mathrm{R}}=\boldsymbol{\lambda}^{\mathrm{L}, \mathrm{R}}+\boldsymbol{\delta}_{N}$ определяет соответствующие строгие разбиения. Благодаря возникновению $F_{\boldsymbol{\mu}^{\mathrm{L}} ; \boldsymbol{\mu}^{\mathrm{R}}}(\beta)$ в $(122)$, корреляционная функция выживания ферромагнитной струны связана с перечислением путей недружественных пешеходов в модели блужданий со случайными поворотами. В пределе $\beta \rightarrow 0$ представление (122) переходит в (91), так как значение правой части (115) дается произведением $\prod_{n=1}^{N} \delta_{\mu_{n} \nu_{n}}$ (соотношение ортогональности). Подставляя (117) в (122) и применяя формулу Бине-Коши (78), получаем [80], [97]:

$$
\begin{aligned}
& \left\langle\Psi_{N}(\mathbf{v})\left|\bar{\Pi}_{n} e^{-\beta \mathscr{H}} \bar{\Pi}_{n}\right| \Psi_{N}(\mathbf{u})\right\rangle \\
& =\frac{1}{(M+1)^{N}} \sum_{\{\boldsymbol{\phi}\}} e^{-\beta E_{N}(\boldsymbol{\phi})}\left|\mathscr{V}_{N}\left(e^{i \boldsymbol{\phi}}\right)\right|^{2} \mathscr{P} \mathscr{M} / n\left(\mathbf{v}^{-2}, e^{i \boldsymbol{\phi}}\right) \mathscr{P} \mathscr{M} / n_{n}\left(e^{-i \boldsymbol{\phi}}, \mathbf{u}^{2}\right) \\
& =\frac{1}{\mathscr{V}_{N}\left(\mathbf{u}^{2}\right) \mathscr{V}_{N}\left(\mathbf{v}^{-2}\right)} \operatorname{det}\left(\sum_{k, l=n}^{M} F_{k ; l}(\beta) \frac{u_{i}^{2 l}}{v_{j}^{2 k}}\right)_{1 \leqslant i, j \leqslant N},
\end{aligned}
$$

где $\phi \equiv \phi_{N}$. Далее, $\mathscr{P}_{L / n}(\mathbf{y}, \mathbf{x})$ и $F_{k ; l}(\beta)$ определены в $(78)$ и $(112)$ соответственно. При $\beta=0$ выражение (124) переходит в (91). При $n=0$ соотношение (124) дает ответ для $\left\langle\Psi_{N}(\mathbf{v})\left|e^{-\beta \mathscr{H}}\right| \Psi_{N}(\mathbf{u})\right\rangle$.

Принимая во внимание соотношение

$$
\left\langle\Psi_{N}(\mathbf{v})\left|e^{-\beta \mathscr{H}}\right| \Psi_{N}\left(e^{i \boldsymbol{\theta} / 2}\right)\right\rangle=\left\langle\Psi_{N}(\mathbf{v}) \mid \Psi_{N}\left(e^{i \boldsymbol{\theta} / 2}\right)\right\rangle e^{-\beta E_{N}(\boldsymbol{\theta})}
$$

где энергия $E_{N}(\boldsymbol{\theta})$ дана в (64), получаем из (124) ответ для выживания ферромагнитной струны (88):

$$
\mathscr{T}\left(\boldsymbol{\theta}_{N}^{\mathrm{g}}, n, \beta\right)=\frac{e^{\beta E_{N}\left(\boldsymbol{\theta}^{\mathrm{g}}\right)}}{(M+1)^{N}} \operatorname{det}\left(\sum_{k, l=n}^{M} F_{k ; l}(\beta) e^{i\left(l \theta_{i}^{\mathrm{g}}-k \theta_{j}^{\mathrm{g}}\right)}\right)_{1 \leqslant i, j \leqslant N},
$$

где энергия основного состояния $E_{N}\left(\boldsymbol{\theta}^{\mathrm{g}}\right)$ дана соотношением (65). Из (123) следует представление для корреляционной функции, которое удобно при изучении асимптотического поведения:

$$
\begin{aligned}
\mathscr{T}\left(\boldsymbol{\theta}_{N}^{\mathrm{g}}, n, \beta\right)= & \frac{1}{\mathscr{N}^{2}\left(\boldsymbol{\theta}^{\mathrm{g}}\right)(M+1)^{N}} \sum_{\{\boldsymbol{\theta}\}} e^{-\beta\left(E_{N}(\boldsymbol{\theta})-E_{N}\left(\boldsymbol{\theta}^{\mathrm{g}}\right)\right)} \\
& \times\left|\mathscr{V}_{N}\left(e^{i \boldsymbol{\theta}}\right) \mathscr{P} \mathscr{M} / n\left(e^{-i \boldsymbol{\theta}}, e^{i \boldsymbol{\theta}^{\mathrm{g}}}\right)\right|^{2}
\end{aligned}
$$

где $\mathscr{N}\left(\boldsymbol{\theta}^{\mathrm{g}}\right)$ обозначает норму (93) для основного состояния (65) и выражение для $\mathscr{P} \mathscr{M} / n\left(e^{-i \boldsymbol{\theta}}, e^{i \boldsymbol{\theta}^{\mathrm{g}}}\right)$ вычисляется с помощью (78) на бетевских решениях (63) и (65). Как видно из (122) и (124), выживание ферромагнитной струны связано с производящими функциями блужданий недружественных пешеходов (110) и (115). 
Перейдем к выводу замкнутого выражения для выживания доменной стенки $\mathscr{F}\left(\boldsymbol{\theta}_{N-n}^{\mathrm{g}}, n, \beta\right)(89)$, аналогичного (126) (см. [80], [97]). Пользуясь в числителе дроби в (89) разложением единицы (94) и применяя (101), получаем:

$$
\begin{aligned}
& \left\langle\Psi_{N-n}(\mathbf{v})\left|\overline{\mathrm{F}}_{n}^{+} e^{-\beta \mathscr{H}} \overline{\mathrm{F}}_{n}\right| \Psi_{N-n}(\mathbf{u})\right\rangle \\
& \quad=\mathrm{D}^{n}(\mathbf{u}) \mathrm{D}^{n}\left(\mathbf{v}^{-1}\right)\left\langle\Psi_{N}(\mathbf{v})\left|e^{-\beta \mathscr{H}}\right| \Psi_{N}(\mathbf{u})\right\rangle,
\end{aligned}
$$

где $\overline{\mathrm{F}}_{n}^{+}$- оператор, эрмитово сопряженный с $\overline{\mathrm{F}}_{n}(89)$ и действующий на сопряженное состояние (77). Кроме того, в правой части (128)

$$
\begin{aligned}
& \mathbf{v}=\mathbf{v}_{N}=\left(\mathbf{v}_{N-n}, v_{N-n+1}, \ldots, v_{N}\right), \\
& \mathbf{u}=\mathbf{u}_{N}=\left(\mathbf{u}_{N-n}, u_{N-n+1}, \ldots, u_{N}\right) .
\end{aligned}
$$

Используя в (128) соотношение (124), взятое при $n=0$, приходим к ответу:

$$
\begin{aligned}
& \left\langle\Psi_{N-n}(\mathbf{v})\left|\overline{\mathrm{F}}_{n}^{+} e^{-\beta \mathscr{H}} \overline{\mathrm{F}}_{n}\right| \Psi_{N-n}(\mathbf{u})\right\rangle=\frac{1}{\mathscr{V}_{N-n}\left(\mathbf{u}^{2}\right) \mathscr{V}_{N-n}\left(\mathbf{v}^{-2}\right)} \\
& \quad \times \mathrm{D}_{v_{N-n+1}^{-1}, v_{N-n+2}^{-1}, \ldots, v_{N}^{-1}} \circ \mathrm{D}_{u_{N-n+1}, u_{N-n+2}, \ldots, u_{N}} \operatorname{det}\left(\sum_{k, l=0}^{M} F_{k ; l}(\beta) \frac{u_{i}^{2 l}}{v_{j}^{2 k}}\right)_{1 \leqslant i, j \leqslant N},
\end{aligned}
$$

где $\mathrm{D}_{u_{N-n+1}, u_{N-n+2}, \ldots, u_{N}}$ задается формулами $(99)$ и $(100)$ и аналогично определяется $\mathrm{D}_{v_{N-n+1}^{-1}, v_{N-n+2}^{-1}, \ldots, v_{N}^{-1}}$. Дифференцирования в (129) выполняются непосредственно, и можно получить [80] формулы, аналогичные соотношениям (124) и (126).

Использование форм-фактора (102) позволяет получить для выживания доменной стенки выражение в терминах функций Шура, аналогичное представлению (127) (см. [80]):

$$
\begin{aligned}
\mathscr{F}\left(\boldsymbol{\theta}_{N-n}^{\mathrm{g}}, n, \beta\right)= & \frac{1}{\mathscr{N}^{2}\left(\boldsymbol{\theta}_{N-n}^{\mathrm{g}}\right)(M+1)^{N-n}} \sum_{\left\{\boldsymbol{\theta}_{N-n}\right\}} e^{-\beta\left(E_{N-n}(\boldsymbol{\theta})-E_{N-n}\left(\boldsymbol{\theta}^{\mathrm{g}}\right)\right)} \\
& \times\left|\mathscr{V}_{N-n}\left(e^{i \boldsymbol{\theta}}\right) \sum_{\boldsymbol{\lambda} \subseteq\left\{\mathscr{M}^{N-n}\right\}} S_{\widehat{\boldsymbol{\lambda}}}\left(e^{-i \boldsymbol{\theta}}\right) S_{\boldsymbol{\lambda}}\left(e^{i \boldsymbol{\theta}^{\mathrm{g}}}\right)\right|^{2}
\end{aligned}
$$

где суммирование идет по всем решениям уравнений Бете $(62)$ и $\boldsymbol{\theta}_{N-n}^{\mathrm{g}}$ соответствует решению (90) для основного состояния из $N-n$ частиц. Кроме того,

$$
\begin{aligned}
E_{N-n}(\boldsymbol{\theta}) & \equiv E_{N-n}\left(\boldsymbol{\theta}_{N-n}\right), & E_{N-n}\left(\boldsymbol{\theta}^{\mathrm{g}}\right) & \equiv E_{N-n}\left(\boldsymbol{\theta}_{N-n}^{\mathrm{g}}\right), \\
e^{i \boldsymbol{\theta}} & \equiv e^{i \boldsymbol{\theta}_{N-n}}, & e^{i \boldsymbol{\theta}^{\mathrm{g}}} & \equiv e^{i \boldsymbol{\theta}_{N-n}^{\mathrm{g}}} .
\end{aligned}
$$

4.2. Форм-факторы и корреляционная функция выживания ферромагнитной струны при бесконечной анизотропии. Перейдем к случаю сильной анизотропии $\Delta \rightarrow-\infty$, который описывается гамильтонианом $\widehat{H}_{\mathrm{SA}}(67)$. Будем вычислять корреляционную функцию выживания ферромагнитной струны, которую, следуя [98], рассмотрим для состояний, не имеющих 
спинов "вниз" на последних $n$ узлах решетки:

$$
\mathscr{T}\left(\boldsymbol{\theta}_{N}^{\mathrm{g}}, n, \beta\right) \equiv \frac{\left\langle\Psi_{N}\left(\boldsymbol{\theta}^{\mathrm{g}}\right)\left|\bar{\Pi}_{n} e^{-\beta \widehat{H}_{\mathrm{SA}}} \bar{\Pi}_{n}\right| \Psi_{N}\left(\boldsymbol{\theta}^{\mathrm{g}}\right)\right\rangle}{\left\langle\Psi_{N}\left(\boldsymbol{\theta}^{\mathrm{g}}\right)\left|e^{-\beta \widehat{H}_{\mathrm{SA}}}\right| \Psi_{N}\left(\boldsymbol{\theta}^{\mathrm{g}}\right)\right\rangle}, \quad \bar{\Pi}_{n} \equiv \prod_{j=M-n+1}^{M} \check{q}_{j},
$$

где $\widehat{H}_{\mathrm{SA}}, \check{q}_{j}$ и $\boldsymbol{\theta}^{\mathrm{g}}$ определены соотношениями (67), (68) и (72) соответственно. Вектор состояния определен соотношением (76). Будем считать $\bar{\Pi}_{0}$ тождественным оператором, т. е. $\mathscr{T}\left(\boldsymbol{\theta}^{\mathrm{g}}, 0, \beta\right)=1$.

Начнем с нормированного среднего (95), числитель которого вычисляется с помощью формулы Бине-Коши:

$$
\begin{aligned}
\left\langle\Psi_{N}(\mathbf{v})\left|\bar{\Pi}_{n}\right| \Psi_{N}(\mathbf{u})\right\rangle & =\sum_{\tilde{\boldsymbol{\lambda}} \subseteq\left\{(M-2 N+2-n)^{N}\right\}} S_{\widetilde{\boldsymbol{\lambda}}}\left(\mathbf{v}^{-2}\right) S_{\widetilde{\boldsymbol{\lambda}}}\left(\mathbf{u}^{2}\right) \\
& =\frac{\operatorname{det}\left(T_{k j}\right)_{1 \leqslant k, j \leqslant N}}{\mathscr{V}_{N}\left(\mathbf{u}^{2}\right)_{N}\left(\mathbf{v}^{-2}\right)},
\end{aligned}
$$

где

$$
T_{k j}=\frac{1-\left(u_{k}^{2} / v_{j}^{2}\right)^{M-N+2-n}}{1-u_{k}^{2} / v_{j}^{2}} .
$$

При $n=0$ соотношение (132) сводится к формуле для скалярного произведения. Скалярное произведение $\left\langle\Psi_{N}(\mathbf{v}) \mid \Psi_{N}(\mathbf{u})\right\rangle$ обращается в нуль, если параметры $\mathbf{u}$ и $\mathbf{v}$ отвечают независимым бетевским решениям [98]. Заметим также, что обозначение $T_{k j}^{\circ}$ в (86) соответствует $T_{k j}(132)$ при $n=0$ (использование $T_{k j}^{\circ}$ в (86) и, скажем, в (107) не противоречиво, так как ясно, какой из пределов анизотропии и какая матрица $T_{k j}$ имеются при этом в виду). Квадрат нормы собственных векторов, отвечающих уравнению Бете $(70)$, $\mathscr{N}^{2}(\boldsymbol{\theta}) \equiv\left\langle\Psi_{N}(\boldsymbol{\theta}) \mid \Psi_{N}(\boldsymbol{\theta})\right\rangle$, выражается соотношением

$$
\mathscr{N}^{2}(\boldsymbol{\theta})=\frac{(M+1)(M+1-N)^{N-1}}{\left|\mathscr{V}_{N}\left(e^{i \boldsymbol{\theta}}\right)\right|^{2}}=\frac{(M+1)(M+1-N)^{N-1}}{\prod_{1 \leqslant m<l \leqslant N} 2\left(1-\cos \left(\theta_{l}-\theta_{m}\right)\right)},
$$

где используется решение (71). Использование (132) и (133) позволяет выразить (95) на бетевских решениях (71) в виде вероятности образования пустоты, аналогичной (96):

$$
\begin{aligned}
E(n, N) \equiv & \frac{M-N+1}{M+1} \operatorname{det}\left(\left(1-\frac{n-1}{M-N+1}\right) \delta_{j k}\right. \\
& \left.+\frac{1-e^{i(n-1)\left(\theta_{j}-\theta_{k}\right)}}{(M-N+1)\left(1-e^{i\left(\theta_{k}-\theta_{j}\right)}\right)}\left(1-\delta_{j k}\right)\right)_{1 \leqslant k, j \leqslant N} .
\end{aligned}
$$

Вычислим числитель (131) в произвольной параметризации:

$$
\begin{aligned}
& \left\langle\Psi_{N}(\mathbf{v})\left|\bar{\Pi}_{n} e^{-\beta \widehat{H}_{\mathrm{SA}}} \bar{\Pi}_{n}\right| \Psi_{N}(\mathbf{u})\right\rangle \\
& =\sum_{\widetilde{\boldsymbol{\lambda}}^{\mathrm{L}}, \widetilde{\boldsymbol{\lambda}}^{\mathrm{R}} \subseteq\left\{(M-2 N+2-n)^{N}\right\}} S_{\widetilde{\boldsymbol{\lambda}}^{\mathrm{L}}}\left(\mathbf{v}^{-2}\right) S_{\widetilde{\boldsymbol{\lambda}}^{\mathrm{R}}}\left(\mathbf{u}^{2}\right) F_{\widetilde{\boldsymbol{\mu}}^{\mathrm{L}} ; \widetilde{\boldsymbol{\mu}}^{\mathrm{R}}}(\beta),
\end{aligned}
$$


где $F_{\widetilde{\boldsymbol{\mu}}^{\mathrm{L}} ; \widetilde{\boldsymbol{\mu}}^{\mathrm{R}}}(\beta)$ есть $2 N$-точечная функция над ферромагнитным состоянием, определяемая формулой (115), где вместо гамильтониана (60) используется гамильтониан $\widehat{H}_{\mathrm{SA}}(67)$. Суммирование по разбиениям $\tilde{\lambda}^{\mathrm{L}}$ и $\tilde{\lambda}^{\mathrm{R}}$ в (134) аналогично суммированию в (76). Соответствующие строгие разбиения $\widetilde{\boldsymbol{\mu}}^{\mathrm{L}}$ и $\widetilde{\boldsymbol{\mu}}^{\mathrm{R}}$ определяются следующим образом: $\widetilde{\boldsymbol{\mu}}^{\mathrm{L}, \mathrm{R}}=\widetilde{\boldsymbol{\lambda}}^{\mathrm{L}, \mathrm{R}}+2 \boldsymbol{\delta}_{N}$, где $\boldsymbol{\delta}_{N}=(N-1, N-2, \ldots, 0)$. Части разбиений $\widetilde{\boldsymbol{\mu}}^{\mathrm{L}}$ и $\widetilde{\boldsymbol{\mu}}^{\mathrm{R}}$ удовлетворяют требованию исключения.

Решениям уравнений Бете (70) соответствует полный набор собственных состояний, и разложение единичного оператора принимает вид

$$
\mathbf{I}=\frac{1}{(M+1)(M+1-N)^{N-1}} \sum_{\{\boldsymbol{\theta}\}}\left|\mathscr{V}_{N}\left(e^{i \boldsymbol{\theta}}\right)\right|^{2}\left|\Psi_{N}\left(e^{i \boldsymbol{\theta} / 2}\right)\right\rangle\left\langle\Psi_{N}\left(e^{i \boldsymbol{\theta} / 2}\right)\right|,
$$

где $\sum_{\{\boldsymbol{\theta}\}}$ означает суммирование по всем независимым решениям (71). Будем вычислять числитель дроби в (131), используя (135). Принимая во внимание соотношение (125), получаем:

$$
\begin{aligned}
\left\langle\Psi_{N}(\mathbf{v})\left|\bar{\Pi}_{n} e^{-\beta \widehat{H}_{\mathrm{SA}}} \bar{\Pi}_{n}\right| \Psi_{N}(\mathbf{u})\right\rangle=\mathrm{V}^{-1} & \sum_{\{\boldsymbol{\theta}\}} e^{-\beta E_{N}^{\mathrm{SA}}(\boldsymbol{\theta})}\left|\mathscr{V}_{N}\left(e^{i \boldsymbol{\theta}}\right)\right|^{2} \\
& \times \widetilde{\mathscr{P}}\left(\mathbf{v}^{-2}, e^{i \boldsymbol{\theta}}\right) \widetilde{\mathscr{P}}\left(e^{-i \boldsymbol{\theta}}, \mathbf{u}^{2}\right),
\end{aligned}
$$

где $\mathrm{V} \equiv(M+1)(M+1-N)^{N-1}$ и $E_{N}^{\mathrm{SA}}(\boldsymbol{\theta})$ - собственное значение энергии при бесконечной анизотропии (73). Суммы

$$
\widetilde{\mathscr{P}}\left(\mathbf{v}^{-2}, e^{i \boldsymbol{\theta}}\right) \equiv \sum_{\widetilde{\boldsymbol{\lambda}}^{\mathrm{L}}} S_{\widetilde{\boldsymbol{\lambda}}^{\mathrm{L}}}\left(\mathbf{v}^{-2}\right) S_{\widetilde{\boldsymbol{\lambda}}^{\mathrm{L}}}\left(e^{i \boldsymbol{\theta}}\right), \quad \widetilde{\mathscr{P}}\left(e^{-i \boldsymbol{\theta}}, \mathbf{u}^{2}\right) \equiv \sum_{\widetilde{\boldsymbol{\lambda}}^{\mathrm{R}}} S_{\widetilde{\boldsymbol{\lambda}}^{\mathrm{R}}}\left(e^{-i \boldsymbol{\theta}}\right) S_{\widetilde{\boldsymbol{\lambda}}^{\mathrm{R}}}\left(\mathbf{u}^{2}\right)
$$

в правой части (136) определены аналогично суммам в (78) и берутся по областям $\tilde{\boldsymbol{\lambda}}^{\mathrm{L}}, \tilde{\boldsymbol{\lambda}}^{\mathrm{R}} \subseteq\left\{(M-2 N+2-n)^{N}\right\}$ соответственно. Тогда, используя (78), мы получаем из (136) выражение для $\mathscr{T}\left(\boldsymbol{\theta}_{N}^{\mathrm{g}}, n, \beta\right)(131)$ :

$$
\mathscr{T}\left(\boldsymbol{\theta}_{N}^{\mathrm{g}}, n, \beta\right)=\mathrm{V}^{-2} \sum_{\{\boldsymbol{\theta}\}} e^{-\beta\left(E_{N}^{\mathrm{SA}}(\boldsymbol{\theta})-E_{N}^{\mathrm{SA}}\left(\boldsymbol{\theta}^{\mathrm{g}}\right)\right)}\left|\operatorname{det} T\left(\boldsymbol{\theta}, \boldsymbol{\theta}^{\mathrm{g}}\right)\right|^{2},
$$

где суммирование идет по всем независимым решениям $\boldsymbol{\theta}(71)$ и $\boldsymbol{\theta}^{\mathrm{g}}$ - решение для основного состояния (72). В представлении $(138) \operatorname{det} T\left(\boldsymbol{\theta}, \boldsymbol{\theta}^{\mathrm{g}}\right)$ обозначает определитель матрицы $\left(T_{k j}\right)_{1 \leqslant k, j \leqslant N}(132)$, вычисленной для $\mathbf{u}^{2}=e^{i \boldsymbol{\theta}}$ и $\mathbf{v}^{2}=e^{i \boldsymbol{\theta}^{\mathrm{g}}}$, и $E_{N}^{\mathrm{SA}}\left(\boldsymbol{\theta}^{\mathrm{g}}\right)$ - энергия основного состояния в пределе сильной анизотропии (74).

\section{5. q-биномиальные определители, решеточные пути и полустандартные таблицы Юнга}

Прежде чем показать, что скалярные произведения векторов состояния и некоторые форм-факторы $X X Z$-цепочки в пределах нулевой и бесконечной анизотропии связаны с производящими функциями плоских разбиений и решеточных самоизбегающих путей, приведем необходимые сведения о производящих 
функциях плоских разбиений [20], [30], [52], а также о q-биномиальных [79] и биномиальных [108] определителях.

\section{1. q-биномиальные определители и определитель купербергов-} ского типа. Итак, перейдем к связи производящих функции плоских разбиений с форм-факторами операторов $\bar{\Pi}_{n}$ и $\overline{\mathrm{F}}_{n}$, вычисленными в специальной $q$-параметризации (42). Продемонстрируем, что форм-факторы (91), (108) и (132), вычисленные в $q$-параметризации (42), связаны с производящими функциями (1), (3) и (6) плоских разбиений в ящике. $\mathrm{K}$ соответствующим формулам приводит теорема 4, которая будет сформулирована в данном разделе и в которой существенно используются q-биномиальные определители [79]. Напомним основные определения.

ОПРЕДЕЛЕНИЕ 4. Пусть $[n] \equiv\left(1-q^{n}\right) /(1-q)$ есть $q$-число, являющееся $q$-аналогом положительного целого $n \in \mathbb{Z}_{+}$, и пусть определены $q$-факториаль $[n] ! \equiv[1][2] \cdots[n]$ и $[0] ! \equiv 1$. Введем пару $S$-наборов, а и $\mathbf{b}$, упорядоченных следующим образом: $0 \leqslant a_{1}<a_{2}<\cdots<a_{S}$ и $0 \leqslant b_{1}<b_{2}<\cdots<b_{S}$. Тогда q-биномиальный определитель, $\left(\begin{array}{l}\mathbf{a} \\ \mathbf{b}\end{array}\right)_{q}$, имеет вид

$$
\left(\begin{array}{l}
\mathbf{a} \\
\mathbf{b}
\end{array}\right)_{q} \equiv\left(\begin{array}{llll}
a_{1}, & a_{2}, & \ldots, & a_{S} \\
b_{1}, & b_{2}, & \ldots, & b_{S}
\end{array}\right)_{q} \equiv \operatorname{det}\left(\left[\begin{array}{c}
a_{j} \\
b_{i}
\end{array}\right]\right)_{1 \leqslant i, j \leqslant S}
$$

где матричные элементы $\left[\begin{array}{l}a_{j} \\ b_{i}\end{array}\right]$ задаются $q$-биномиальными коэффиииентами:

$$
\left[\begin{array}{c}
N \\
r
\end{array}\right] \equiv \frac{[N] !}{[r] ![N-r] !}
$$

Свойства q-биномиальных коэффициентов (140) приведены в приложении А. В пределе $q \rightarrow 1$-биномиальные коэффициенты становятся биномиальными коэффициентами $\left(\begin{array}{c}a_{j} \\ b_{i}\end{array}\right)$ и q-биномиальный определитель (139) превращается в биномиальный определитель [108]

$$
\left(\begin{array}{l}
\mathbf{a} \\
\mathbf{b}
\end{array}\right) \equiv\left(\begin{array}{llll}
a_{1}, & a_{2}, & \ldots, & a_{S} \\
b_{1}, & b_{2}, & \ldots, & b_{S}
\end{array}\right)=\operatorname{det}\left(\left(\begin{array}{c}
a_{j} \\
b_{i}
\end{array}\right)\right)_{1 \leqslant i, j \leqslant S} .
$$

Биномиальный определитель (141) неотрицателен и становится положительным, если $b_{i} \leqslant a_{i}$ для всех $i$ [108]. Упорядочение элементов а и $\mathbf{b}$ в (139), (141) согласовано с работой [108], где показано, что биномиальный определитель (141) дает число самоизбегающих путей определенного типа на двумерной решетке. А именно, соответствующий набор решеточных путей $\left(w_{1}, w_{2}, \ldots, w_{S}\right)$ таков, что путь $w_{i}$ идет из $A_{i}=\left(0, a_{i}\right)$ в $B_{i}=\left(b_{i}, b_{i}\right), 1 \leqslant i \leqslant S$, и при этом допустимы шаги только в северном и восточном направлениях. Типичный набор изображен на рис. 9 для $S=5$. 


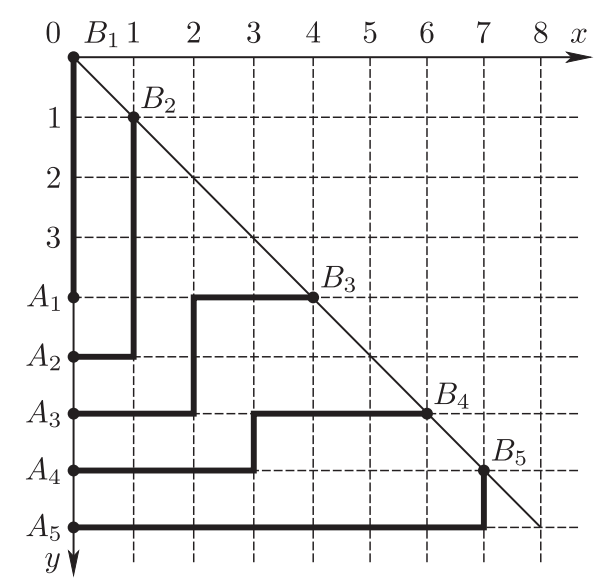

Рис. 9. $S$-набор $\left(w_{1}, w_{2}, \ldots, w_{S}\right)$ самоизбегающих путей для $S=5$

Основным утверждением данного раздела является следующая теорема.

ТЕОРема 4. Пусть q-биномиальный определитель $\mathrm{B}_{q}$ задан формулой

$$
\begin{aligned}
\mathrm{B}_{q} & \equiv \operatorname{det}\left(\left[\begin{array}{c}
N+L+i-1 \\
L+j-1
\end{array}\right]\right)_{1 \leqslant i, j \leqslant \mathscr{P}} \\
& =\left(\begin{array}{cccc}
N+L, & N+L+1, & \ldots, & N+L+\mathscr{P}-1 \\
L, & L+1, & \ldots, & L+\mathscr{P}-1
\end{array}\right)_{q} \\
& =\left(\begin{array}{cccc}
L+N, & L+1+N, & \ldots, & L+P \\
L, & L+1, & \ldots, & L+P-N
\end{array}\right)_{q}
\end{aligned}
$$

и матричные элементы (106) вычислены в q-параметризации (42):

$$
\begin{array}{lll}
\overline{\mathrm{T}}_{k j}=\frac{1-q^{(P+1)(j+k-1)}}{1-q^{j+k-1}}, & 1 \leqslant k \leqslant L, & 1 \leqslant j \leqslant N, \\
\overline{\mathrm{T}}_{k j}=q^{j(N-k)}, & L+1<k \leqslant N, & 1 \leqslant j \leqslant N,
\end{array}
$$

где $P / 2<N<P, L \leqslant N$ (числа $P$ и $L$ в (143) произвольны; если $L=N$, то матрица $\bar{T}$ состоит из одного блока). Тогда значение определителя матриць $(\overline{\mathbf{T}})_{1 \leqslant j, k \leqslant N}(143)$ выражается из следующих соотношений:

$$
\begin{aligned}
q^{-L(L-1)(N-L) / 2} \frac{\operatorname{det}(\overline{\mathbf{T}})_{1 \leqslant j, k \leqslant N}}{\mathscr{V}_{N}(\mathbf{q}) \mathscr{V}_{L}(\mathbf{q} / q)} & =q^{N(1-\mathscr{P}) \mathscr{P} / 2} \mathrm{~B}_{q} \\
& =\prod_{k=1}^{\mathscr{P}} \prod_{j=1}^{L} \frac{1-q^{j+k+N-1}}{1-q^{j+k-1}}=Z_{q}(L, N, \mathscr{P}),
\end{aligned}
$$

где $\mathscr{P} \equiv P-N+1$ и $\mathscr{V}_{N}(\mathbf{q}), \mathscr{V}_{L}(\mathbf{q} / q)$ - определители Вандермонда. Кроме того, $Z_{q}(L, N, \mathscr{P})$ есть производящая функиия (1) плоских разбиений в $\mathscr{B}(L, N, \mathscr{P})$.

ДокАЗАТЕЛЬСтво соотношений (144) и (145) (где $1 \leqslant L \leqslant N$ ), основанное на теории симметрических функций, приведено в приложении А. При $L=0$ 
можно формально записать:

$$
\operatorname{det} \overline{\mathrm{T}}=q^{-N(\mathscr{P}-1) \mathscr{P} / 2 \mathscr{V}_{N}(\mathbf{q})}\left(\begin{array}{cccc}
N, & N+1, & \ldots, & N+\mathscr{P}-1 \\
0, & 1, & \ldots, & \mathscr{P}-1
\end{array}\right)_{q},
$$

где значение $\left.\mathrm{B}_{q}\right|_{L=0}=q^{N(\mathscr{P}-1) \mathscr{P} / 2} q$-биномиального определителя получается из (А.17). Таким образом, определитель $\operatorname{det} \overline{\mathrm{T}}$ сводится к определителю Вандермонда. Теорема 4 доказана.

ЗАмечАниЕ 1. Уместно называть определитель матрицы Т (143) определителем куперберговского типа (см. [28], где обсуждается связь числа знакопеременных матриц [30] со статистической суммой шестивершинной модели с граничным условием типа доменной стенки). А именно, в случае $L=N$ значение $\operatorname{det} \overline{\mathrm{T}}$, даваемое теоремой 4 , согласуется с (53) при $s=q^{P+1}$. Отличие состоит в том, что матрица $\overline{\mathrm{T}}(143)$ состоит из двух блоков и ее матричные элементы зависят от одного параметра $q$, тогда как матрица под знаком определителя в левой части (53) состоит из одного блока, зависящего от двух независимых параметров $s$ и $q$. Доказательство теоремы 4, в отличие от доказательства в [28], основано на формуле Бине-Коши и теории симметрических функций.

\section{2. Производящая функция конфигурации “арбуз" и полустан-} дартные таблицы Юнга. В данном пункте представлена наглядная комбинаторная интерпретация, связанная с основным утверждением теоремы 4. А именно, будет показано, что основные соотношения (144) и (145) связаны с конфигурациями решеточных путей типа "арбуз" [44]. В работах [48]-[50] конфигурации решеточных путей "арбуз" и "звезда" обсуждались в связи со случайными блужданиями недружественных пешеходов.

5.2.1. Конфигурация решеточных путей “арбуз". Итак, соотношение (144) связывает $\operatorname{det} \overline{\mathrm{T}}$ с q-биномиальным определителем (142), который переходит при $q \rightarrow 1$ в биномиальный определитель, равный числу $\mathscr{P}$-наборов самоизбегающих решеточных путей, соединяющих конщевые точки $A_{l}=(0, N+L+l-1)$ и $B_{l}=(L+l-1, L+l-1), 1 \leqslant l \leqslant \mathscr{P}$. Рис. 9 дает изображение соответствующих путей, но при этом точки $A_{l}$ и $B_{l}$, связанные с $\mathrm{B}_{q}(142)$, эквидистантны. Так как узлы $A_{l}$ на оси ординат являются ближайшими, то соответствующие непересекающиеся решеточные пути имеют горизонтальные участки, примыкающие к $A_{l}$ (см. рис. 10 для $\mathscr{P}=L=3$ и $N=2$ ).

Конфигурация "арбуз" [44], [48]-[50] отвечает набору самоизбегающих путей между точками $C_{l}=(l-1, N+L+l-1)$ и $B_{l}=(L+l-1, L+l-1)$, $1 \leqslant l \leqslant \mathscr{P}$, и возникает, как показано на рис. 10 , благодаря частичной "ампутации" горизонтальных участков путей, примыкающих к точкам $A_{l}$. Концевые точки путей, образующих “арбуз", располагаются эквидистантно. Кроме того, каждый путь характеризуется одними и теми же полными числами шагов, подсчитанными независимо вдоль осей абсцисс и ординат. Например, для "арбуза", задаваемого точками $C_{l}=(l-1, N+L+l-1)$ и $B_{l}=(L+l-1, L+l-1)$, $1 \leqslant l \leqslant \mathscr{P}$, полные числа шагов вдоль осей абсцисс и ординат равны $L$ и $N$ соответственно. 

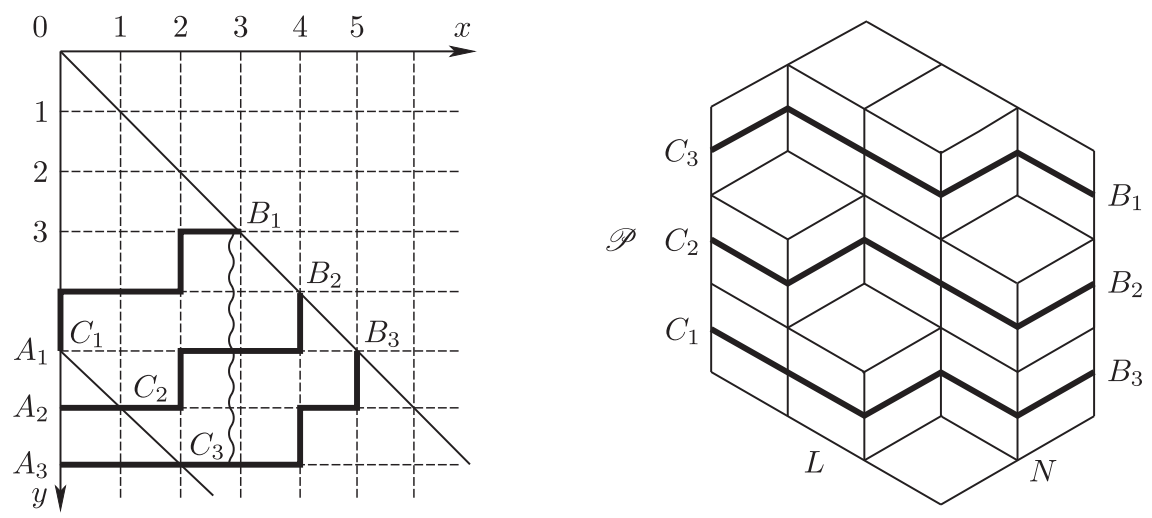

Рис. 10. Набор самоизбегающих решеточных путей, образующих конфигурацию "арбуз" (слева), и трехмерная диаграмма Юнга с градиентными линиями

В пределе $q \rightarrow 1$ производящая функция $Z_{q}(L, N, \mathscr{P})(145)$ дает число плоских разбиений $A(L, N, \mathscr{P})(2)$ в ящике $\mathscr{B}(L, N, \mathscr{P})$ (формула Мак-Магона [43]):

$$
\begin{aligned}
Z_{q}(L, N, \mathscr{P}) & =\prod_{j=1}^{L} \prod_{k=1}^{N} \frac{1-q^{\mathscr{P}+j+k-1}}{1-q^{j+k-1}} \\
& \underset{q \rightarrow 1}{\longrightarrow} A(L, N, \mathscr{P})=\operatorname{det}\left(\left(\begin{array}{c}
N+L+i-1 \\
L+j-1
\end{array}\right)\right)_{1 \leqslant i, j \leqslant \mathscr{P}} .
\end{aligned}
$$

Правое равенство в формуле (146) выражает совпадение числа плоских разбиений $A(L, N, \mathscr{P})$ и числа самоизбегающих решеточных путей (см. рис. 10). При этом именно для путей, образующих "арбуз", имеет место биективное соответствие с градиентными линиями на трехмерной диаграмме Юнга, изображающей плоское разбиение в $\mathscr{B}(L, N, \mathscr{P})$.

Биективность соответствия между плоскими разбиениями в ящике и "арбузами" [48] приводит к комбинаторной интерпретации равенства

$$
q^{-N(\mathscr{P}-1) \mathscr{P} / 2}\left(\begin{array}{cccc}
L+N, & L+N+1, & \ldots, & L+N+\mathscr{P}-1 \\
L, & L+1, & \ldots, & L+\mathscr{P}-1
\end{array}\right)_{q}=Z_{q}(L, N, \mathscr{P}) .
$$

Напомним [19], [30], что биномиальный коэффициент вида $\left(\begin{array}{c}n+m \\ m\end{array}\right)$ равен числу решеточных путей внутри прямоугольника размера $n \times m$, левая нижняя и правая верхняя вершины которого совпадают с точками $(0,0)$ и $(n, m)$ соответственно. Шаги совершаются только вверх или вправо. Множество единичных квадратов сверху и слева от такого пути образует диаграмму Юнга некоторого разбиения $\boldsymbol{\lambda}$, для которого $l(\boldsymbol{\lambda}) \leqslant m$ и $\lambda_{1} \leqslant n$. Число путей из $(0,0)$ в $(n, m)$ равно числу способов разместить диаграмму Юнга в прямоугольнике. Производящая функция таких диаграмм дается $q$-биномиальным коэффициентом $\left[\begin{array}{c}n+m \\ m\end{array}\right]=\sum_{\{\boldsymbol{\lambda}\}} q^{|\boldsymbol{\lambda}|}$. Таким образом, вклады диаграмм Юнга 
в соответствующие $q$-биномиальные коэффициенты, образующие (142), порождаются весами $q^{|\boldsymbol{\lambda}|}[19]$. Веса путей между $A_{l}=(0, N+L+l-1)$ и $B_{l}=$ $(L+l-1, L+l-1)$ содержат общий множитель, отличающий их от весов путей между $C_{l}=(l-1, N+L+l-1)$ и $B_{l}=(L+l-1, L+l-1), 1 \leqslant l \leqslant \mathscr{P}$. Данный множитель имеет вид

$$
q^{\sum_{k=1}^{\mathscr{P}}\left|\boldsymbol{\lambda}^{\prime}(k)\right|}=q^{N(\mathscr{P}-1) \mathscr{P} / 2},
$$

где $\boldsymbol{\lambda}^{\prime}(k)$ обозначает нестрогие разбиения $(k-1, k-1, \ldots, k-1), 1 \leqslant k \leqslant \mathscr{P}$, длина и объем которых суть $l\left(\boldsymbol{\lambda}^{\prime}(k)\right)=N$ и $\left|\boldsymbol{\lambda}^{\prime}(k)\right|=N(k-1)$ соответственно. Конфигурация на рис. 10 приводит к множителю $q^{2 \times 0+2 \times 1+2 \times 2}=q^{6}$, согласующемуся с формулой (148). Другими словами, равенство (147) между $Z_{q}(L, N, \mathscr{P})$ и произведением $q^{-N(\mathscr{P}-1) \mathscr{P} / 2}$ на определитель $\mathrm{B}_{q}(142)$ оказывается соотношением между производящими функциями плоских разбиений и "арбузов" соответственно.

Пусть типичная конфигурация "арбуз" образована путями с концевыми точками $C_{l}$ и $B_{l}, 1 \leqslant l \leqslant \mathscr{P}$ (рис. 10 ). Путь с номером $l$ содержится в прямоугольнике, левая нижняя и правая верхняя вершины которого даны точками $C_{l}$ и $B_{l}$ соответственно. Так как "арбузы" тесно связаны с плоскими разбиениями в ящике [19], определим объем пути как число клеток, расположенных под путем внутри соответствующего прямоугольника, и объем конфигурации “арбуз" как суммарный объем всех путей, образующих "арбуз".

Нам потребуется следующее определение.

ОПРЕДЕЛЕНИЕ 5. Производящая функиия $\mathrm{W}_{q}(L, N)$ конфигуращии “арбуз”, характеризующейся полными числами шагов $L$ и $N$ вдоль осей абсцисс и ординат, дается соотношением

$$
\mathrm{W}_{q}(L, N) \equiv \sum_{\left\{\mathbf{w}_{L N}\right\}} q^{\left|\mathbf{w}_{L N}\right|}
$$

где $\sum_{\left\{\mathbf{w}_{L N}\right\}}$ означает суммирование по всем наборам $\mathbf{w}_{L N}$ путей, образующих “арбуз". Кроме того, $\left|\mathbf{w}_{L N}\right|$ - объем конфигурации $\mathbf{w}_{L N}$ и $q^{\left|\mathbf{w}_{L N}\right|}$ - соответствующий статистический вес.

5.2.2. Полустандартные таблицы Юнга. Доказательство соотношений (144) и (145), содержащееся в приложении А, основано на связи функций Шура с элементарными симметрическими функциями. В основе доказательства лежит соотношение (А.9), которое следует из утверждений теоремы 3, записанных в $q$-параметризации. Объединяя утверждения теоремы 3 и теоремы 4, можно сформулировать следующий результат.

ПреДЛОЖЕНИЕ 6. Положим $L=N$ u $P=M+N-1$ в (144), (145) $u$ учтем (А.9). Тогда имеет место соотношение

$$
\begin{aligned}
\sum_{\boldsymbol{\lambda} \subseteq\left\{M^{N}\right\}} S_{\boldsymbol{\lambda}}(\mathbf{q}) S_{\boldsymbol{\lambda}}\left(\frac{\mathbf{q}}{q}\right) & =q^{N M(1-M) / 2} \operatorname{det}\left(\left[\begin{array}{c}
2 N+i-1 \\
N+j-1
\end{array}\right]\right)_{1 \leqslant i, j \leqslant M} \\
& =\prod_{k=1}^{N} \prod_{j=1}^{N} \frac{1-q^{M+j+k-1}}{1-q^{j+k-1}} .
\end{aligned}
$$


Соотношение (150) является частным случаем равенства (147) и будет в данном пункте выведено с использованием связи между функциями Шура и полустандартнъми таблицами Юнга, допускающими, в свою очередь, интерпретацию в терминах самоизбегающих путей. Использование полустандартных таблиц позволяет выразить сумму в левой части (150) в терминах производящей функции (149). Независимое вычисление функции (149) приводит ко второму соотношению в (150). Иными словами, для соотношения (150) возникает "графическое" (в отличие от независимого "алгебраического" в приложении А) доказательство, позволяющее пояснить возникновение конфигурации "арбуз".

Идея "графического" доказательства состоит в следующем. Конфигурацию "арбуз" можно "рассечь" (как схематически показано на рис. 10 с помощью вертикальной волнистой линии) и получить два набора путей (для обозначения которых будем в дальнейшем использовать буквы $\mathscr{B}$ и $\mathscr{C}$ ). При этом суммирования по конфигурациям "арбуз" эквивалентны независимым суммированиям по наборам путей $\mathscr{B}$ и $\mathscr{C}$. Кроме того, для обоих наборов $\mathscr{B}$ и $\mathscr{C}$ концевые точки, возникающие при "рассечении", являются в общем случае неэквидистантными. Как следствие, каждому набору путей, соединяющих эквидистантные точки с неэквидистантными, можно естественным образом поставить в соответствие (полустандартную) таблицу Юнга, отвечающую разбиению, части которого связаны с нумерацией неэквидистантных узлов.

Итак, дадим следующее определение.

ОПРЕдЕЛЕНИЕ 6 . Пусть разбиение $\boldsymbol{\lambda}$ отождествлено с соответствующей диаграммой Юнга. Тогда полустандартной таблищей Юнга $T$ на диаграмме $\boldsymbol{\lambda}$ называется диаграмма, клетки которой заполнены положительными целыми числами $n \in \mathbb{N}$, слабо убывающими по строкам и строго убывающими по столбцам.

Пусть $\mathbf{x} \equiv\left(x_{1}, \ldots, x_{N}\right)$, где $N=l(\boldsymbol{\lambda})$. Определим вес таблицы $T$ как произведение $\mathbf{x}^{T} \equiv \prod_{i, j} x_{T_{i j}}$, взятое по всем элементам $T_{i j}$ таблицы $T$. Тогда для функции Шура выполняется следующее, эквивалентное определению (35), соотношение [94]:

$$
S_{\boldsymbol{\lambda}}(\mathbf{x})=\sum_{\{T\}} \mathbf{x}^{T},
$$

где суммирование идет по всем таблицам $T$ на диаграмме $\boldsymbol{\lambda}$, содержащим в клетках натуральные числа от 1 до $N$.

ДоКАЗАТЕЛЬСтво ПРЕДЛОЖЕНИЯ 6. Шаг І. Пусть $T$ - полустандартная таблица на диаграмме $\boldsymbol{\lambda}$, отвечающей разбиению длины $l(\boldsymbol{\lambda})=N$. Определим набор $\mathscr{C}$ самоизбегающих путей, которые соединяют расположенные эквидистантно точки $C_{i}=(N-i+1, N-i), 1 \leqslant i \leqslant N$, с неэквидистантными точками $\left(1, \mu_{i}\right)$, где $\mu_{i}=\lambda_{i}+N-i$ (см. рис. 11). ${ }^{2}$ В наборе $\mathscr{C}$ каждый $i$-й путь, отсчитываемый сверху вниз, содержит $\lambda_{i}$ шагов на север. При этом шагов на север вдоль линии узлов с абсциссой $j$ имеется столько, сколько раз число $N-j+1$

\footnotetext{
${ }^{2}$ Направление и нумерация абсцисс и ординат выбраны с учетом правила заполнения клеток полустандартных таблиц Юнга [19].
} 


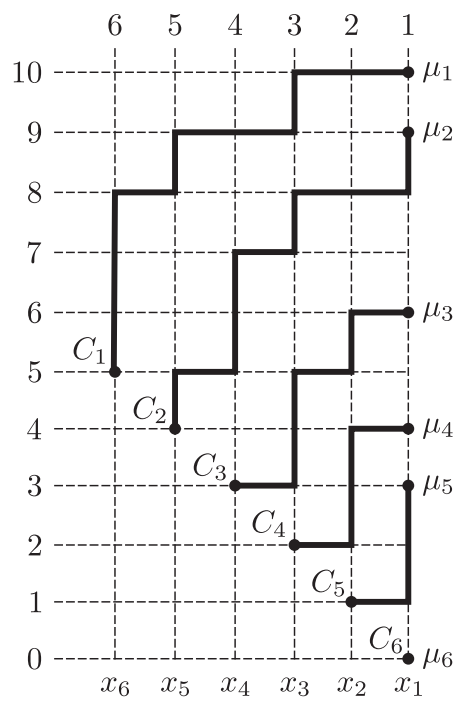

\begin{tabular}{|c|c|c|c|c|}
\hline 1 & 1 & 1 & 2 & 4 \\
\hline 2 & 3 & 3 & 4 & 6 \\
\hline 4 & 4 & 5 & & \\
\hline 5 & 5 & & & \\
\hline 6 & 6 & & & \\
\hline
\end{tabular}

Рис. 11. Набор решеточных путей $\mathscr{C}$, соответствующих полустандартной таблице с весом $x_{6}^{3} x_{5}^{2} x_{4}^{2} x_{3}^{4} x_{2}^{3} x_{1}^{3}$ на диаграмме $\boldsymbol{\lambda}=(5,5,3,2,2,0)$ для $N=6$

встречается в клетках $i$-й строки таблицы $T$. Для каждой таблицы $T$ вес $\mathbf{x}^{T}$ принимает вид одночлена $\prod_{j=1}^{N} x_{j}^{c_{j}}$, где $c_{j}$ равно количеству появлений числа $N-j+1$ во всей таблице. Следовательно, представление (151) принимает вид

$$
S_{\boldsymbol{\lambda}}(\mathbf{x})=\sum_{\{\mathscr{C}\}} \prod_{j=1}^{N} x_{j}^{c_{j}}
$$

где суммирование идет по всем допустимым наборам $\mathscr{C}$ и в каждом наборе полное число шагов на север вдоль $j$-й абсциссы равно $c_{j}$. Представление $(152)$ естественно возникает в моделях, решаемых с помощью КМО3 [109].

Путь с номером $i$ содержится в прямоугольнике размера $\lambda_{i}(N-i), 1 \leqslant i \leqslant N$. Объем каждого пути равен числу квадратов, расположенных под ним внутри соответствующего прямоугольника. Объем набора путей $|\mathscr{C}|$ равен суммарному объему отдельных путей:

$$
|\mathscr{C}|=\sum_{j=1}^{N}(j-1) c_{j}
$$

Из соотношений (152) и (153) видно, что функция Шура с аргументом в $q$-параметризованном виде $\mathbf{x}=\mathbf{q} / q \equiv\left(1, q, \ldots, q^{N-1}\right)$ принимает вид статистической суммы: $S_{\boldsymbol{\lambda}}(\mathbf{q} / q)=\sum_{\{\mathscr{C}\}} q^{|\mathscr{C}|}$, где суммирование идет по всем допустимым набо- 


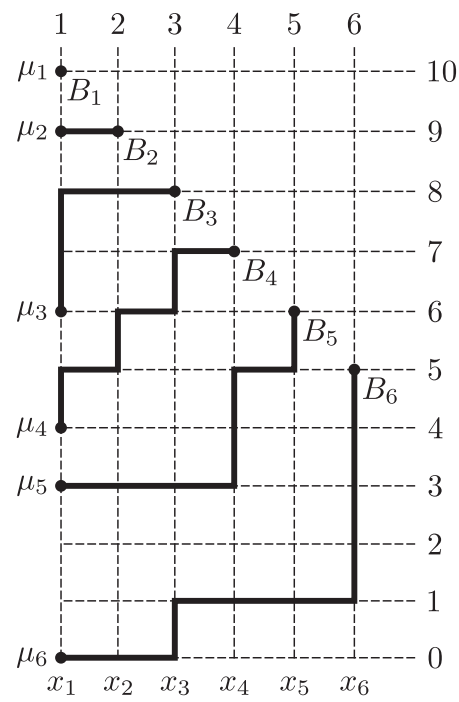

Рис. 12. Сопряженный набор решеточных путей $\mathscr{B}$ для таблицы Юнга на диаграмме $\mathbf{M}-\boldsymbol{\lambda}$ с весом $x_{1}^{2} x_{2}^{4} x_{3}^{3} x_{4}^{3} x_{5}^{4} x_{6}$ для $N=6$ и $M=5$

рам $\mathscr{C}$. Добавляя вес разбиения $|\boldsymbol{\lambda}|$ к объему набора, получаем:

$$
S_{\boldsymbol{\lambda}}(\mathbf{q})=\sum_{\{\mathscr{C}\}} q^{|\boldsymbol{\lambda}|+|\mathscr{C}|}=q^{|\boldsymbol{\lambda}|} S_{\boldsymbol{\lambda}}\left(\frac{\mathbf{q}}{q}\right),
$$

где $|\boldsymbol{\lambda}|+|\mathscr{C}|=\sum_{j=1}^{N} j c_{j}$, так как $|\boldsymbol{\lambda}|$ совпадает с $\sum_{j=1}^{N} c_{j}$.

Рассмотрим сопряженный набор самоизбегающих путей $\mathscr{B}$, которые соединяют точки $\left(1, \mu_{i}\right)$, где $\mu_{i}=\lambda_{i}+N-i, 1 \leqslant i \leqslant N$, с эквидистантными точками $B_{i}=(i, N+M-i)$ (рис. 12$)$. Конфигурация $\mathscr{B}$ описывается полустандартной таблицей на диаграмме $\mathbf{M}-\boldsymbol{\lambda}$, где $\mathbf{M} \equiv(M, M, \ldots, M)$ и $l(\mathbf{M})=N$. При этом соответствующий $i$-й путь делает $M-\lambda_{i}$ шагов на север. Полустандартной таблице отвечает функция Шура (см. [52])

$$
S_{\boldsymbol{\lambda}}(\mathbf{x})=\sum_{\{\mathscr{B}\}} \prod_{j=1}^{N} x_{j}^{M-b_{j}},
$$

где $b_{j}$ - полное число шагов на север вдоль линии узлов с абсциссой $j$ и суммирование идет по всем допустимым наборам $\mathscr{B}$. Путь с номером $i$ содержится в прямоугольнике размера $M(i-1), i=1, \ldots, N$. Объем пути равен числу квадратов под ним внутри соответствующего прямоугольника, а объем набора путей равен суммарному объему всех путей. Тогда

$$
S_{\boldsymbol{\lambda}}\left(\frac{\mathbf{q}}{q}\right)=\sum_{\{\mathscr{B}\}} q^{|\mathscr{B}|}, \quad|\mathscr{B}|=\sum_{j=1}^{N}(j-1)\left(M-b_{j}\right) .
$$


С учетом (154) и (156) получаем, что значение функции Шура при $q \rightarrow 1$ (см. соотношение (А.22) в приложении А) равно числу наборов самоизбегающих решеточных путей типа $\mathscr{B}$ или $\mathscr{C}$ :

$$
S_{\boldsymbol{\lambda}}(1,1, \ldots, 1)=\sum_{\{\mathscr{B}\}} 1=\sum_{\{\mathscr{C}\}} 1 .
$$

Соединим диаграммы, изображенные на рис. 11 и рис. 12 , по точкам, задаваемым разбиением $\boldsymbol{\mu}$ на линии "разреза", так, чтобы получилась конфигурация “арбуз", образованная набором путей с концевыми точками $C_{i}$ и $B_{i}, i=1, \ldots, N$ (на рис. 13 представлена конфигурация "арбуз" для $N=6$ и $M=5$ ). Благодаря графическому соответствию сумма произведений функций Шура в левой части (150) равна сумме по $N$-наборам самоизбегающих путей, которые соединяют точки $C_{i}$ и $B_{i}(i=1, \ldots, N)$, т. е. сумма в $(150)$ сводится к сумме по всем "арбузам". Объем "арбуза" есть $\left|\mathbf{w}_{N M}\right|=|\mathscr{C}|+|\mathscr{B}|$, и с учетом (149) получаем, что левая часть (150) равна производящей функции $\mathrm{W}_{q}(N, M)$.

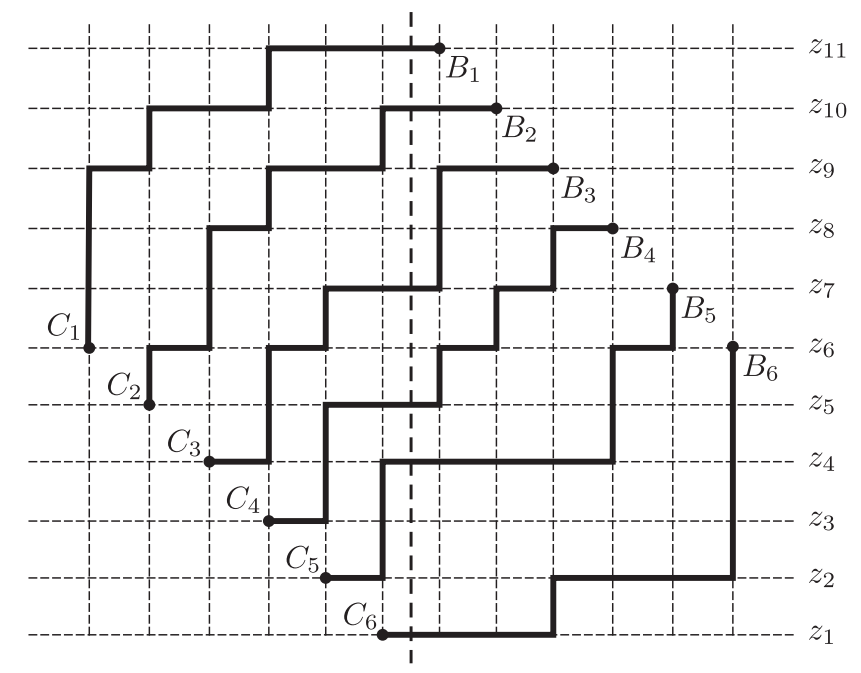

Рис. 13. Конфигурация "арбуз"

Шаг II. Конфигурации "арбуз" с концевыми точками $C_{i}$ и $B_{i}(i=1, \ldots, N)$ отвечает соответствующая полустандартная таблица Юнга, размещенная в прямоугольнике размера $N \times N$. Для рассматриваемой конфигурации “арбуз" каждый путь характеризуется $N$ шагами на восток и $M$ шагами на север. Пусть $m_{j}$ обозначает число шагов на восток вдоль ординаты $z_{j}, j=1, \ldots, N+M($ см. рис. 13). Запишем соответствующую функцию Шура в виде

$$
S_{\mathbf{N}}\left(z_{1}, z_{2}, \ldots, z_{N+M}\right)=\sum_{\left\{\mathbf{w}_{N M}\right\}} \prod_{j=1}^{N+M} z_{j}^{m_{j}},
$$

где $\mathbf{N}$ есть нестрогое разбиение

$$
\mathbf{N}=(\underbrace{N, N, \ldots, N}_{N} \underbrace{0,0, \ldots, 0}_{M})
$$


длины $l(\mathbf{N})=N+M$ и суммирование идет по всем допустимым "арбузам". Объем "арбуза" имеет вид

$$
\left|\mathbf{w}_{N M}\right|=-\frac{N^{2}(N-1)}{2}+\sum_{j=1}^{M+N}(j-1) m_{j} .
$$

Статистическая сумма “арбузов” выражается через функцию Шура (157):

$$
\mathrm{W}_{q}(N, M)=q^{-N^{2}(N-1) / 2} S_{\mathbf{N}}\left(1, q^{2}, \ldots, q^{N+M-1}\right),
$$

значение которой вычисляется с помощью известной формулы (см. [52; гл. 1.3, пример 1])

$$
S_{\boldsymbol{\lambda}}\left(1, q^{2}, \ldots, q^{m-1}\right)=q^{\sum_{i=1}^{m}(i-1) \lambda_{i}} \prod_{1 \leqslant i<j \leqslant m} \frac{1-q^{\lambda_{i}-\lambda_{j}-i+j}}{1-q^{j-i}} .
$$

Кроме того, если $m>N$, то $\lambda_{i}=0$ для $i>N$. Тогда из (158) и (159) получаем:

$$
\mathrm{W}_{q}(N, M)=\prod_{i=1}^{N} \prod_{j=N+1}^{N+M} \frac{1-q^{N-i+j}}{1-q^{j-i}} .
$$

Делая в (160) замены $j \mapsto N+j$ и $i \mapsto N+1-i$, приходим ко второму соотношению в (150):

$$
\mathrm{W}_{q}(N, M)=\prod_{i=1}^{N} \prod_{j=1}^{M} \frac{1-q^{N+i+j-1}}{1-q^{j+i-1}}=\prod_{i=1}^{N} \prod_{j=1}^{N} \frac{1-q^{M+i+j-1}}{1-q^{j+i-1}} .
$$

Соотношение (161) выражает производящую функцию “арбузов" $\mathrm{W}_{q}(N, M)$ через производящие функции $Z_{q}(N, M, N)$ и $Z_{q}(N, N, M)$ плоских разбиений в ящике (см. переход от (А.18) к (А.19) в приложении А). Эквивалентность

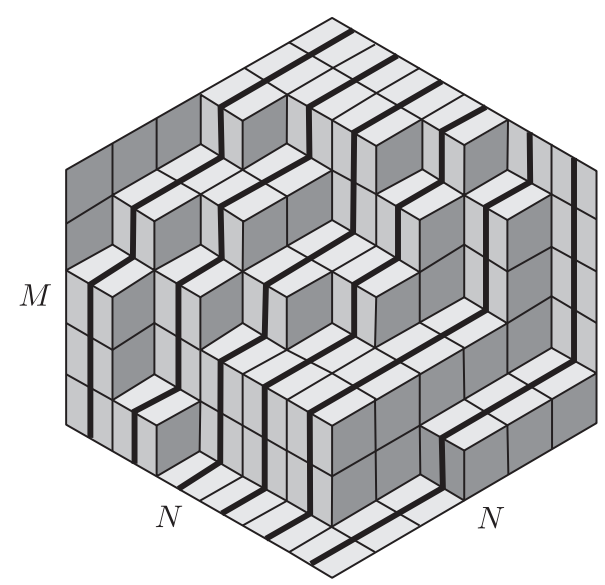

Рис. 14. Трехмерная диаграмма Юнга, отвечающая конфигурации “арбуз" 
между $Z_{q}(N, M, N)$ и $Z_{q}(N, N, M)$ существенна при доказательстве как теоремы 4, так и рассматриваемого предложения 6. На рис. 14 изображена трехмерная диаграмма Юнга с градиентными линиями, которые взаимно однозначно соответствуют путям конфигурации “арбуз” на рис. 13. Равенство производящих функций $Z_{q}(N, M, N)$ и $Z_{q}(N, N, M)$ отражает тот геометрический факт, что трехмерную диаграмму Юнга, которая "визуализирует" укладку элементарных кубиков, можно рассматривать, вообще говоря, с трех независимых направлений (более подробно см. [30]). Наконец, из соотношений (149) и (161) следует (150). Предложение 6 доказано.

\section{6. Форм-факторы и асимптотики корреляционных функций $X X Z$-магнетика при нулевой и бесконечной анизотропии}

\section{1. Форм-факторы в $q$-параметризации и производящие функции} плоских разбиений. Перейдем к вычислению форм-факторов в $q$-параметризации (42). Начнем с форм-фактора (91) ферромагнитной струны. Значения матричных элементов (92) в $q$-параметризации (42) связаны с матричными элементами (143) при $L=N$ и $P=M-n$. В результате, используя (144) и (145), приходим к ответу:

$$
\begin{aligned}
& \left\langle\Psi_{N}\left(\mathbf{q}^{-1 / 2}\right)\left|\bar{\Pi}_{n}\right| \Psi_{N}\left(\left(\frac{\mathbf{q}}{q}\right)^{1 / 2}\right)\right\rangle=\mathscr{P}_{\mathscr{M} / n}\left(\mathbf{q}, \frac{\mathbf{q}}{q}\right) \\
& =q^{n N^{2}} \prod_{k=1}^{N} \prod_{j=1}^{N} \frac{1-q^{M-n+1+j-k}}{1-q^{j+k-1}}=q^{N^{2}(2 n+1-N) / 2} Z_{q}^{\operatorname{cspp}}(N, N, M-n) .
\end{aligned}
$$

Соотношение (162) выражает форм-фактор через производящую функцию строгих по столбцам разбиений в ящике $\mathscr{B}(N, N, M-n)$. В пределе $q \rightarrow 1$ возникает число $A^{\mathrm{cspp}}(N, N, M-n)(4)$ строгих по столбцам разбиений:

$$
\lim _{q \rightarrow 1}\left\langle\Psi_{N}\left(\mathbf{q}^{-1 / 2}\right)\left|\bar{\Pi}_{n}\right| \Psi_{N}\left(\left(\frac{\mathbf{q}}{q}\right)^{1 / 2}\right)\right\rangle=A^{\operatorname{cspp}}(N, N, M-n) .
$$

В случае сильной анизотропии пользуемся выражением (132) для форм-фактора оператора $\bar{\Pi}_{n}$ и получаем:

$$
\lim _{q \rightarrow 1}\left\langle\Psi_{N}\left(\mathbf{q}^{-1 / 2}\right)\left|\bar{\Pi}_{n}\right| \Psi_{N}\left(\left(\frac{\mathbf{q}}{q}\right)^{1 / 2}\right)\right\rangle=A^{\mathrm{spp}}(N, N, M-n),
$$

где $A^{\mathrm{spp}}(N, N, M-n)$ - число строгих плоских разбиений в ящике $\mathscr{B}(N, N, M-n)$ (см. (8)).

Выражение $\mathscr{P} \mathscr{\mathscr { M } / n}(\mathbf{q}, \mathbf{q} / q)$ в (162) есть $q$-параметризованная сумма (78). Используя представления (А.21), можно выразить функции Шура в этой сумме через определители, параметризованные сопряженными разбиениями $\overline{\boldsymbol{\mu}}$ (сопряженные разбиения используются в приложении А при доказательстве теоремы 4). Тогда с учетом (5), (144) и (145) соотношение (162) (пусть $n=0$ для наглядности) приводит при $q \rightarrow 1$ к следующему представлению для числа 
плоских разбиений в ящике $\mathscr{B}(N, N, \mathscr{M})$ :

$$
\begin{aligned}
A(N, N, \mathscr{M}) & =\left(\begin{array}{cccc}
2 N, & 2 N+1, & \ldots, & 2 N+\mathscr{M}-1 \\
N, & N+1, & \ldots, & N+\mathscr{M}-1
\end{array}\right) \\
& =\sum_{\bar{\lambda} \subseteq\left\{N^{\mathscr{M}}\right\}}\left(\begin{array}{cccc}
N, & N+1, & \ldots, & N+\mathscr{M}-1 \\
\bar{\lambda}_{\mathscr{M}}, & \bar{\lambda}_{\mathscr{M}-1}+1, & \ldots, & \bar{\lambda}_{1}+\mathscr{M}-1
\end{array}\right)^{2} .
\end{aligned}
$$

Соотношение (165) представляет число плоских разбиений в форме, демонстрирующей связь с наборами самоизбегающих путей, концевые точки которых проиндексированы сопряженными разбиениями (см. биномиальные определители (А.22) в приложении А). Как показано в [108], определитель (А.22) дает число $\mathscr{M}$-наборов решеточных путей, связывающих точки $A_{l}=(0, N+l-1)$ и $B_{l}=\left(\bar{\mu}_{\mathscr{M}+1-l}, \bar{\mu}_{\mathscr{M}+1-l}\right), 1 \leqslant l \leqslant \mathscr{M}$. Представление $(165)$ аналогично представлению (150) для производящей функции “арбузов" (предложение 6).

Далее, форм-фактор $\left\langle\Psi_{N}\left(\mathbf{q}^{-1 / 2}\right)\left|\overline{\mathrm{F}}_{n}\right| \Psi_{N-n}\left((\mathbf{q} / q)^{1 / 2}\right)\right\rangle$, где подразумевается, что $\mathbf{q}^{-1 / 2}=\left(q^{-1 / 2}, q^{-1}, \ldots, q^{-N / 2}\right)$ и $(\mathbf{q} / q)^{1 / 2}=\left(1, q^{1 / 2}, \ldots, q^{(N-n-1) / 2}\right)$, связан с производящей функцией плоских разбиений в $\mathscr{B}(N-n, N, \mathscr{M})$. Используя (102) и (104), получаем в q-параметризации:

$$
\begin{aligned}
& \left\langle\Psi_{N}\left(\mathbf{q}^{-1 / 2}\right)\left|\bar{F}_{n}\right| \Psi_{N-n}\left(\left(\frac{\mathbf{q}}{q}\right)^{1 / 2}\right)\right\rangle \\
& =q^{n(N-n)(N-n-1) / 2} \sum_{\lambda \subseteq\left\{\mathscr{M}^{N-n}\right\}} S_{\widehat{\boldsymbol{\lambda}}}(\mathbf{q}) S_{\boldsymbol{\lambda}}\left(\frac{\mathbf{q}}{q}\right)=\frac{\operatorname{det} \overline{\mathbf{T}}}{\mathscr{V}_{N}(\mathbf{q}) \mathscr{V}_{N-n}(\mathbf{q} / q)},
\end{aligned}
$$

где $\overline{\mathrm{T}}$ задается формулами (143) при $L=N-n$ и $P=M$. Разбиения $\widehat{\boldsymbol{\lambda}}$ и $\boldsymbol{\lambda}$ определены одинаково для (102) и для (166). Используя (145), из (166) получаем:

$$
\left\langle\Psi_{N}\left(\mathbf{q}^{-1 / 2}\right)\left|\overline{\mathrm{F}}_{n}\right| \Psi_{N-n}\left(\left(\frac{\mathbf{q}}{q}\right)^{1 / 2}\right)\right\rangle=q^{n(N-n)(N-n-1) / 2} Z_{q}(N-n, N, \mathscr{M}) .
$$

Таким образом,

$$
\lim _{q \rightarrow 1}\left\langle\Psi_{N}\left(\mathbf{q}^{-1 / 2}\right)\left|\overline{\mathrm{F}}_{n}\right| \Psi_{N-n}\left(\left(\frac{\mathbf{q}}{q}\right)^{1 / 2}\right)\right\rangle=A(N-n, N, \mathscr{M})
$$

и среднее $\left\langle\Psi_{N}\left(\mathbf{q}^{-1 / 2}\right)\left|\overline{\mathrm{F}}_{n}\right| \Psi_{N-n}\left((\mathbf{q} / q)^{1 / 2}\right)\right\rangle$ есть производящая функция формулы Мак-Магона (2) для плоских разбиений в ящике $\mathscr{B}(N-n, N, \mathscr{M})$.

\section{2. Асимптотики корреляционных функций при убывающей тем-} пературе. Представляет интерес получить приближенные выражения для корреляционных функций (88), (89) и (131) при $1 \ll N \ll M$ и $T \ll 1$. В случае корреляционной функции (88) воспользуемся представлением (127), которое эквивалентно (126) (см. [80], [97]). В указанном пределе решения (63) заполняют при достаточно больших $M$ отрезок длины $2 \pi$, в результате чего переход в $(127)$ 
от сумм к интегралам позволяет получить оценку

$$
\begin{aligned}
\mathscr{T}\left(\boldsymbol{\theta}_{N}^{\mathrm{g}}, n, \beta\right) \simeq & \frac{1}{\mathscr{N}^{2}\left(\boldsymbol{\theta}^{\mathrm{g}}\right) N !} \int_{-\pi}^{\pi} \int_{-\pi}^{\pi} \ldots \int_{-\pi}^{\pi} \exp \left\{\beta \sum_{l=1}^{N}\left(\cos \theta_{l}-1\right)\right\} \\
& \times\left|\mathscr{P}_{\mathscr{M} / n}\left(e^{-i \boldsymbol{\theta}}, \mathbf{1}\right)\right|^{2} \prod_{1 \leqslant k<l \leqslant N}\left|e^{i \theta_{k}}-e^{i \theta_{l}}\right|^{2} \frac{d \theta_{1} d \theta_{2} \cdots d \theta_{N}}{(2 \pi)^{N}},
\end{aligned}
$$

где учтено, что $\cos \theta_{l}^{\mathrm{g}} \simeq 1$ для решения (65). Интеграл в правой части (169) аналогичен статистической сумме классического газа заряженных частиц на единичной окружности с логарифмическим потенциалом $\log \left|e^{i \theta_{k}}-e^{i \theta_{l}}\right|$ (см. [82]).

Аппроксимируем $\mathscr{T}\left(\boldsymbol{\theta}_{N}^{\mathrm{g}}, n, \beta\right)(169)$ при $\beta \gg 1$ (низкотемпературный предел), вынося предельное значение $\lim _{q \rightarrow 1} \mathscr{P} \mathscr{M} / n(\mathbf{q}, \mathbf{q} / q)$ из под знака интегрирования:

$$
\begin{gathered}
\mathscr{T}\left(\boldsymbol{\theta}_{N}^{\mathrm{g}}, n, \beta\right) \simeq \frac{\mathscr{A}(N, n)}{\beta^{N^{2} / 2}}, \\
\mathscr{A}(N, n) \equiv\left(A^{\mathrm{cspp}}(N, N, M-n)\right)^{2} \frac{\mathscr{I}_{N}}{\mathscr{N}^{2}\left(\boldsymbol{\theta}^{\mathrm{g}}\right)},
\end{gathered}
$$

где $\mathscr{I}_{N}$ - интеграл Мехты (120). Как следует из (162), предельное значение

$$
\lim _{q \rightarrow 1} \mathscr{P}_{\mathscr{M} / n}\left(\mathbf{q}, \frac{\mathbf{q}}{q}\right)
$$

совпадает с числом $A^{\operatorname{cspp}}(N, N, M-n)$ разбиений, строгих по столбцам.

Выражение для интеграла $\mathscr{I}_{N}(120)$, содержащее гамма-функции, удобно представить в экспоненциальном виде:

$$
\mathscr{I}_{N}=e^{\varphi_{N}}, \quad \varphi_{N} \equiv \sum_{k=1}^{N} \log \frac{\Gamma(k)}{(2 \pi)^{1 / 2}} .
$$

В рассматриваемом пределе обратная величина квадрата нормы имеет вид

$$
\frac{1}{\mathscr{N}^{2}\left(\boldsymbol{\theta}^{\mathrm{g}}\right)} \simeq \frac{(2 \pi)^{N(N-1)}}{(M+1)^{N^{2}}} \prod_{1 \leqslant r<s \leqslant N}|r-s|^{2}=\left(\frac{2 \pi}{M+1}\right)^{N^{2}} e^{2 \varphi_{N}}
$$

где $\varphi_{N}$ определено в (172). С учетом (172) и (173) находим, что соотношение (170) принимает приближенный вид

$$
\mathscr{T}\left(\boldsymbol{\theta}_{N}^{\mathrm{g}}, n, \beta\right) \simeq\left(A^{\operatorname{cspp}}(N, N, M-n)\right)^{2} e^{\Phi(N, M, \beta)},
$$

где

$$
\Phi(N, M, \beta) \equiv N^{2} \log \frac{2 \pi}{M+1}-\frac{N^{2}}{2} \log \beta+3 \varphi_{N} .
$$

Для изучения асимптотических свойств корреляционных функций удобно выразить $\varphi_{N}(172)$ через $G$-функцию Барнса [110]:

$$
G(z+1)=(2 \pi)^{z / 2} \exp \left\{\frac{-z}{2}(z+1)-\frac{\gamma}{2} z^{2}\right\} \prod_{n=1}^{\infty}\left(1+\frac{z}{n}\right)^{n} \exp \left\{-z+\frac{z^{2}}{2 n}\right\}
$$


где $\gamma$ - постоянная Эйлера [91]. Значения $G$-функции для неотрицательных целых значений аргумента $n$ имеют вид

$$
G(n+1)=\frac{(n !)^{n}}{1^{1} 2^{2} \cdots n^{n}}=\prod_{k=1}^{n} \Gamma(k)
$$

Свойства функции Барнса обсуждаются в [111]. Используя (177), переписываем $\varphi_{N}(172)$ в виде

$$
\varphi_{N}=\log G(N+1)-\frac{N}{2} \log (2 \pi)
$$

и, таким образом, значение интеграла (120) выражаем через $G$-функцию (176):

$$
\mathscr{I}_{N}=\frac{G(N+1)}{(2 \pi)^{N / 2}}
$$

При $z \rightarrow \infty$ справедливо асимптотическое равенство

$$
\log G(z+1)=-\log \mathscr{A}+\frac{z}{2} \log (2 \pi)+\left(\frac{z^{2}}{2}-\frac{1}{12}\right) \log z-\frac{3 z^{2}}{4}+\mathscr{O}\left(\frac{1}{z}\right)
$$

(значение постоянной $\mathscr{A}$ приведено в [110], [111]). Поведение $\varphi_{N}$ при $N \gg 1$ следует из (178) и (180):

$$
\varphi_{N}=\frac{N^{2}}{2} \log N-\frac{3 N^{2}}{4}+\mathscr{O}(\log N), \quad N \gg 1
$$

Таким образом, для показателя (175) получаем приближенное равенство

$$
\Phi(N, M, \beta) \simeq N^{2} \log \left(\mathrm{A} \frac{N^{3 / 2}}{M \beta^{1 / 2}}\right)
$$

где А - постоянная. Чтобы оценить $A^{\operatorname{cspp}}(N, N, M-n)$, в (4) положим $P=M-n$ и выразим произведения гамма-функций с помощью (177):

$$
A^{\operatorname{cspp}}(N, N, M-n)=\frac{G^{2}(N+1) G(M+2-n+N) G(M+2-n-N)}{G(2 N+1) G^{2}(M+2-n)} .
$$

Тогда, учитывая (180), находим в ведущем порядке:

$$
\log A^{\operatorname{cspp}}(N, N, M-n) \simeq N^{2} \log \left(\mathrm{B} \frac{M-n}{N}\right), \quad M-n \gg N \gg 1,
$$

где В - некоторая постоянная. С помощью (184) можно получить асимптотическое поведение числа плоских разбиений, строгих по столбцам, в высоком ящике с квадратным дном $\mathscr{B}(N, N, M-n)$. 
Окончательно, принимая во внимание (182) и (184), придаем асимптотическому выражению (174) для выживания ферромагнитной струны следующий вид:

$$
\log \mathscr{T}\left(\boldsymbol{\theta}_{N}^{\mathrm{g}}, n, \beta\right) \simeq N^{2} \log \left(\mathrm{C} \frac{(M-n)^{2}}{M(N \beta)^{1 / 2}}\right)
$$

где $\mathrm{C}=\mathrm{AB}^{2}$. Если $M$ и $N$ растут, а температура $T$ убывает, то из (185) следует, что $\mathscr{T}\left(\boldsymbol{\theta}_{N}^{\mathrm{g}}, n, \beta\right)$ убывает при соблюдении неравенства $T<\frac{1}{\mathrm{C}^{2}} \frac{N M^{2}}{(M-n)^{4}}$.

Представление (130) для корреляционной функции выживания доменной стенки также допускает вышеприведенные действия. В результате получаем следующее асимптотическое равенство:

$$
\mathscr{F}\left(\boldsymbol{\theta}_{N-n}^{\mathrm{g}}, n, \beta\right) \simeq A^{2}(N-n, N, M-N+1) e^{\Phi(N, M, \beta)}
$$

где показатель $\Phi(N, M, \beta)$ определен в $(175)$ и $A(N-n, N, M-N+1)$ есть число плоских разбиений (2) в ящике с прямоугольным дном $\mathscr{B}(N-n, N, M-N+1)$, возникающее как форм-фактор оператора рождения доменной стенки (168).

Записывая соотношение (2) в виде произведений гамма-функций и применяя равенство (177), получаем

$$
\begin{aligned}
A(N-n, N, M-N+1)=\frac{G(N+1) G(N-n+1)}{G(2 N-n+1)} & \\
& \times \frac{G(M+2-n+N) G(M+2-N)}{G(M+2-n) G(M+2)} .
\end{aligned}
$$

Далее, с использованием (180) находим асимптотическое представление

$$
\begin{gathered}
\log A(N-n, N, M-N+1) \simeq N(N-n) \log \left(\mathrm{D} \frac{M-n}{2 N-n}\right), \\
M-n \gg N-n, \quad N \gg 1
\end{gathered}
$$

где D - некоторая постоянная. Соотношение (187) определяет асимптотическое поведение числа плоских разбиений в высоком ящике с прямоугольным дном $\mathscr{B}(N-n, N, M-N+1)$. С учетом (182) и (187) приближенное выражение для (186) принимает вид:

$$
\log \mathscr{F}\left(\boldsymbol{\theta}_{N-n}^{\mathrm{g}}, n, \beta\right) \simeq N^{2} \log \left(\mathrm{A} \frac{N^{3 / 2}}{M \beta^{1 / 2}}\right)+2 N(N-n) \log \left(\mathrm{D} \frac{M-n}{2 N-n}\right)
$$

Из соотношения (188) можно получить, что коррелятор $\mathscr{F}\left(\boldsymbol{\theta}_{N-n}^{\mathrm{g}}, n, \beta\right)$ убывает при возрастании $M$ и $N$, если для температуры $T$ соблюдается оценка, аналогичная полученной выше для (185). 
В изинговском пределе с использованием (136) получаем коррелятор выживания ферромагнитной струны в следующем виде:

$$
\mathscr{T}\left(\boldsymbol{\theta}_{N}^{\mathrm{g}}, n, \beta\right)=\frac{\left|\mathscr{V}_{N}\left(e^{i \boldsymbol{\theta}^{\mathrm{g}}}\right)\right|^{2}}{\mathrm{~V}^{2}} \sum_{\{\boldsymbol{\theta}\}} e^{-\beta\left(E_{N}^{\mathrm{SA}}(\boldsymbol{\theta})-E_{N}^{\mathrm{SA}}\left(\boldsymbol{\theta}^{\mathrm{g}}\right)\right)}\left|\mathscr{V}_{N}\left(e^{i \boldsymbol{\theta}}\right) \widetilde{\mathscr{P}}\left(e^{-i \boldsymbol{\theta}}, e^{i \boldsymbol{\theta}^{\mathrm{g}}}\right)\right|^{2}
$$

где $\mathrm{V} \equiv(M+1)(M+1-N)^{N-1}$, и решение для основного состояния дается соотношением (72). Кроме того, $\left|\widetilde{\mathscr{P}}\left(e^{-i \boldsymbol{\theta}}, e^{i \boldsymbol{\theta}^{\mathrm{g}}}\right)\right|^{2}$ выражается с помощью сумм (137).

При соблюдении сильного неравенства $1 \ll N \ll M$ мы можем перейти в (189) от сумм к интегралам. Используя при больших $\beta$ соотношения $\cos \theta_{l} \simeq 1$ и $\cos \theta_{l}^{\mathrm{g}} \simeq 1$ для всех $l$ и принимая во внимание (133), приходим к приближенному равенству:

$$
\mathscr{T}\left(\boldsymbol{\theta}_{N}^{\mathrm{g}}, n, \beta\right) \simeq \frac{\left(A^{\mathrm{spp}}(N, N, M-n)\right)^{2}}{\beta^{N^{2} / 2}} \frac{\mathscr{I}_{N}}{\mathscr{N}^{2}\left(\boldsymbol{\theta}^{\mathrm{g}}\right)},
$$

где $\mathscr{I}_{N}$ - интеграл Мехты $(120)$ и число $A^{\operatorname{spp}}(N, N, M-n)$ строгих плоских разбиений в ящике дается соотношением (8). Далее, оценка (190) принимает вид

$$
\mathscr{T}\left(\boldsymbol{\theta}_{N}^{\mathrm{g}}, n, \beta\right) \simeq\left(A^{\mathrm{spp}}(N, N, M-n)\right)^{2} e^{\Phi(N, M, \beta)}
$$

где $\Phi(N, M, \beta)$ определено в (175).

Таким образом, как следует из (170), (186) и (190), корреляционные функции (88) и (89) асимптотически связаны с комбинаторными соотношениями. При этом, благодаря возникновению в предельных соотношениях $q$-биномиальных определителей, корреляционные функции и соответствующие форм-факторы демонстрируют связь с перечислением плоских разбиений и самоизбегающих решеточных путей [80]. Показатели экспонент в (174), (186) и (191) имеют вид свободной энергии при слабой связи некоторых решеточных калибровочных моделей, в которых был предсказан фазовый переход третьего рода [87]-[90].

\section{7. Заключение}

В обзоре представлен основанный на теории симметрических функций подход к вычислению температурных корреляционных функций интегрируемых квантовых моделей. Подробно исследована $X X Z$-модель Гейзенберга для предельных значений параметра анизотропии. Показано, что такие понятия перечислительной комбинаторики, как самоизбегающие решеточные пути и плоские разбиения, естественно возникают при исследовании форм-факторов модели, а также при изучении асимптотического поведения ее корреляционных функций.

Ответы для корреляционных функций представлены в виде определителей. Задача вычисления форм-факторов операторов в специальной q-параметризации, естественной при анализе асимптотического поведения корреляторов, сводится к вычислению q-биномиального определителя, являющегося производящей функцией плоских разбиений в ящике конечного размера. Размеры 
данного ящика и тип плоских разбиений определяются параметрами модели и видом рассматриваемого оператора. В пределе $q \rightarrow 1$ возникают биномиальные определители, приводящие к интерпретации полученных форм-факторов в терминах самоизбегающих путей на решетке.

В случае цепочки конечной длины получены выражения для температурных корреляционных функций типа выживания ферромагнитной струны и выживания доменной стенки. Показано, что для достаточно длинной цепочки (и умеренного числа перевернутых спинов) корреляционные функции сводятся при низкой температуре к матричным интегралам теории случайных матриц.

Разработанная в нашей статье техника подходит для вычисления температурных и временных корреляционных функций, представляющих интерес как в теории конденсированного состояния, так и в теории квантовых вычислений. Также интересно отметить, что "модель квадратного льда", которая описывается шестивершинной моделью, была реализована экспериментально [112].

Авторы выражают признательность Л.Д. Фаддееву за внимание к работе, а также А. М. Вершику и П. П. Кулишу за полезные обсуждения.

\section{Приложение А. Вычисление определителя куперберговского типа}

Докажем соотношения (144) и (145), используя формулу Бине-Коши [96] и учитывая связь симметрических функиий [52] с q-исчислением [113]. Пусть $q$-число $[n]$ есть $q$-аналог положительного целого $n \in \mathbb{Z}^{+}$:

$$
[n] \equiv \frac{1-q^{n}}{1-q}
$$

и пусть $[n]$ ! есть q-факториал:

$$
[n] ! \equiv[1][2] \cdots[n], \quad[0] ! \equiv 1 .
$$

С помощью (А.1) и (А.2) определяем q-биномиалъный коэффициент $\left[\begin{array}{c}N \\ r\end{array}\right]$ :

$$
\left[\begin{array}{c}
N \\
r
\end{array}\right] \equiv \frac{[N][N-1] \cdots[N-r+1]}{[r] !}=\frac{[N] !}{[r] ![N-r] !},
$$

который называется также гауссовым биномиальным коэфбиииентом и определяет число $r$-мерных подпространств $N$-мерного векторного пространства над полем из $q$ элементов [114]. В пределе $q \rightarrow 1$-биномиальный коэффициент $\left[\begin{array}{c}N \\ r\end{array}\right]$ становится биномиальным коэффициентом $\left(\begin{array}{c}N \\ r\end{array}\right)$.

Существуют два аналога формулы Паскаля для q-биномиальных коэффициентов:

$$
\begin{aligned}
& {\left[\begin{array}{c}
N \\
r
\end{array}\right]=\left[\begin{array}{c}
N-1 \\
r-1
\end{array}\right]+q^{r}\left[\begin{array}{c}
N-1 \\
r
\end{array}\right],} \\
& {\left[\begin{array}{c}
N \\
r
\end{array}\right]=q^{N-r}\left[\begin{array}{c}
N-1 \\
r-1
\end{array}\right]+\left[\begin{array}{c}
N-1 \\
r
\end{array}\right],}
\end{aligned}
$$


где $1 \leqslant r \leqslant N-1$. Для $q$-биномиальных коэффициентов имеет место $q$-сверткка Вандермонда:

$$
\left[\begin{array}{c}
N+N^{\prime} \\
r
\end{array}\right]=\sum_{j=0}^{\min (r, N)} q^{(N-j)(r-j)}\left[\begin{array}{c}
N \\
j
\end{array}\right]\left[\begin{array}{c}
N^{\prime} \\
r-j
\end{array}\right]
$$

Теперь определим элементарные симметрические функиии $e_{r}=e_{r}(\mathbf{x})$, зависящие от $N$ переменных $\mathbf{x}=\left(x_{1}, x_{2}, \ldots, x_{N}\right)$ :

$$
e_{r} \equiv \sum_{i_{1}<i_{2}<\cdots<i_{r}} x_{i_{1}} x_{i_{2}} \cdots x_{i_{r}}
$$

Функции $e_{r}($ А.6) возникают как коэффициенты в произведении

$$
\left(1+t x_{1}\right)\left(1+t x_{2}\right) \cdots\left(1+t x_{N}\right)=1+e_{1} t+e_{2} t^{2}+\cdots+e_{N} t^{N} .
$$

Введем специальные обозначения для функций $e_{r}$ при $\mathbf{x}=\mathbf{q}$ и $\mathbf{x}=\mathbf{q} / q: R_{r} \equiv$ $e_{r}(\mathbf{q} / q)$ и $L_{r} \equiv e_{r}(\mathbf{q})$. Известны следующие значения [52]:

$$
\begin{aligned}
& R_{r}(N) \equiv e_{r}\left(\frac{\mathbf{q}_{N}}{q}\right)=q^{r(r-1) / 2}\left[\begin{array}{c}
N \\
r
\end{array}\right], \\
& L_{r}(N) \equiv e_{r}\left(\mathbf{q}_{N}\right)=q^{r(r+1) / 2}\left[\begin{array}{c}
N \\
r
\end{array}\right],
\end{aligned}
$$

где используются $q$-биномиальные коэффициенты (А.3).

Рассмотрим нестрогое разбиение $\boldsymbol{\lambda}$ :

$$
\mathscr{P} \geqslant \lambda_{1} \geqslant \lambda_{2} \geqslant \cdots \geqslant \lambda_{N} \geqslant 0
$$

где $\mathscr{P} \equiv P-N+1$. Введем, следуя [52], следующее понятие.

ОПРЕДЕЛЕНиЕ А.1. Сопряженное разбиение $\bar{\lambda}$ есть разбиение, диаграмма которого является транспонированием диаграммы Юнга, представляющей $\boldsymbol{\lambda}$ (см. рис. 1). Разбиение $\overline{\boldsymbol{\lambda}}$ состоит из $\mathscr{P}$ частей, $N \geqslant \bar{\lambda}_{1} \geqslant \bar{\lambda}_{2} \geqslant \cdots \geqslant \bar{\lambda}_{\mathscr{P}} \geqslant 0$, и часть $\bar{\lambda}_{k}$ для любого $k$ равна количеству элементов в $k$-й строке диаграммы. Части соответствующего строгого разбиения $\overline{\boldsymbol{\mu}}$ задаются соотношением $\overline{\boldsymbol{\mu}}=$ $\overline{\boldsymbol{\lambda}}+\boldsymbol{\delta}_{\mathscr{P}}$ и удовлетворяют неравенствам $P \geqslant \bar{\mu}_{1}>\bar{\mu}_{2}>\cdots>\bar{\mu}_{\mathscr{P}} \geqslant 0$.

Сопряженные разбиения позволяют выразить функции Шура (35), зависящие от нестрогих разбиений $\boldsymbol{\lambda}$, в терминах элементарных симметрических функций (А.6):

$$
S_{\boldsymbol{\lambda}}(\mathbf{x})=\operatorname{det}\left(e_{\bar{\lambda}_{i}-i+j}(\mathbf{x})\right)_{1 \leqslant i, j \leqslant \mathscr{P}} .
$$

Чтобы охватить утверждения (144) и (145), мы воспользуемся формулой суммирования (104), чтобы выразить определитель $\operatorname{det} \overline{\mathrm{T}}$, записанный в $q$-параметризации (42):

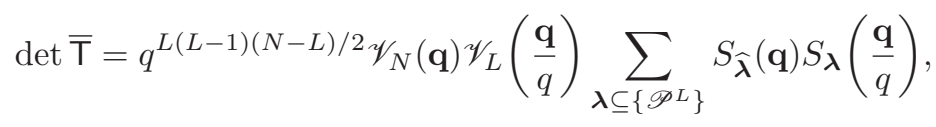


где

$$
\begin{array}{lll}
\overline{\mathrm{T}}_{k j}=\frac{1-q^{(P+1)(j+k-1)}}{1-q^{j+k-1}}, & 1 \leqslant k \leqslant L, & 1 \leqslant j \leqslant N, \\
\overline{\mathrm{T}}_{k j}=q^{j(N-k)}, & L+1 \leqslant k \leqslant N, & 1 \leqslant j \leqslant N .
\end{array}
$$

Введем обозначение $\Sigma_{S}$ для суммы произведений функций Шура в (А.9). Применяя (A.8), преобразуем $\Sigma_{S}$ к сумме по сопряженным разбиениям:

$$
\Sigma_{\mathrm{S}}=\sum_{\overline{\boldsymbol{\lambda} \subseteq\left\{L^{\mathscr{P}}\right\}}} \operatorname{det}\left(e_{\bar{\lambda}_{j}-j+k}\left(\mathbf{q}_{N}\right)\right)_{1 \leqslant j, k \leqslant \mathscr{P}} \operatorname{det}\left(e_{\bar{\lambda}_{p}-p+l}\left(\frac{\mathbf{q}_{L}}{q}\right)\right)_{1 \leqslant l, p \leqslant \mathscr{P}},
$$

где части сопряженного разбиения $\bar{\lambda}$ удовлетворяют следующим неравенствам: $L \geqslant \bar{\lambda}_{1} \geqslant \bar{\lambda}_{2} \geqslant \cdots \geqslant \bar{\lambda}_{\mathscr{P}} \geqslant 0$. Перепишем $\Sigma_{\mathrm{S}}$ в более явном виде:

$$
\begin{array}{r}
\Sigma_{\mathrm{S}}=\sum_{\bar{\lambda} \subseteq\left\{L^{\mathscr{P}}\right\}} \operatorname{det}\left(\begin{array}{cccc}
L_{\bar{\lambda}_{1}}(N) & L_{\bar{\lambda}_{2}-1}(N) & \ldots & L_{\bar{\lambda}_{\mathscr{P}}-\mathscr{P}+1}(N) \\
L_{\bar{\lambda}_{1}+1}(N) & L_{\bar{\lambda}_{2}}(N) & \ldots & L_{\bar{\lambda}_{\mathscr{P}}-\mathscr{P}+2}(N) \\
\vdots & \vdots & \ddots & \vdots \\
L_{\bar{\lambda}_{1}+\mathscr{P}-1}(N) & L_{\bar{\lambda}_{2}+\mathscr{P}-2}(N) & \ldots & L_{\bar{\lambda}_{\mathscr{P}}}(N)
\end{array}\right) \\
\times \operatorname{det}\left(\begin{array}{cccc}
R_{\bar{\lambda}_{1}}(L) & R_{\bar{\lambda}_{1}+1}(L) & \ldots & R_{\bar{\lambda}_{1}+\mathscr{P}-1}(L) \\
R_{\bar{\lambda}_{2}-1}(L) & R_{\bar{\lambda}_{2}}(L) & \ldots & R_{\bar{\lambda}_{2}+\mathscr{P}-2}(L) \\
\vdots & \vdots & \ddots & \vdots \\
R_{\bar{\lambda}_{\mathscr{P}}-\mathscr{P}+1}(L) & R_{\bar{\lambda}_{\mathscr{P}}-\mathscr{P}+2}(L) & \ldots & R_{\bar{\lambda}_{\mathscr{P}}}(L)
\end{array}\right)
\end{array}
$$

где учтены значения (А.7). Кроме того, $R_{0}=L_{0}=1$ (по определению), в то время как $R_{r}$ и $L_{r}$ равны нулю при $r>N$. Формула Бине-Коши позволяет выразить сумму (А.12) в виде определителя матрицы размера $\mathscr{P} \times \mathscr{P}$ :

$\Sigma_{\mathrm{S}}=\operatorname{det}\left(\begin{array}{cccc}\sum_{i=0}^{L} L_{i}(N) R_{i}(L) & \sum_{i=0}^{L} L_{i}(N) R_{i+1}(L) & \ldots & \sum_{i=0}^{L} L_{i}(N) R_{i+\mathscr{P}-1}(L) \\ \sum_{i=0}^{L} L_{i+1}(N) R_{i}(L) & \sum_{i=0}^{L} L_{i}(N) R_{i}(L) & \ldots & \sum_{i=0}^{L} L_{i}(N) R_{i+\mathscr{P}-2}(L) \\ \ldots & \ldots & \ddots & \ldots \\ \sum_{i=0}^{L} L_{i+\mathscr{P}-1}(N) R_{i}(L) & \sum_{i=0}^{L} L_{i+\mathscr{P}-2}(N) R_{i}(L) & \ldots & \sum_{i=0}^{L} L_{i}(N) R_{i}(L)\end{array}\right)$.

Матричные элементы в (А.13) вычисляются с помощью (А.5) и (А.7):

$$
\begin{aligned}
& \sum_{i=0}^{L} L_{i+s}(N) R_{i}(L)=q^{s(s+1) / 2}\left[\begin{array}{c}
N+L \\
N-s
\end{array}\right], \\
& \sum_{i=0}^{L} L_{i}(N) R_{i+s}(L)=q^{s(s-1) / 2}\left[\begin{array}{c}
N+L \\
N+s
\end{array}\right],
\end{aligned}
$$

где $0 \leqslant s \leqslant \mathscr{P}-1$. Используя суммы (А.14), представим (А.13) в виде определителя матрицы с элементами, выраженными с помощью $q$-биномиальных 
коэффициентов:

$$
\Sigma_{\mathrm{S}}=\operatorname{det}\left(q^{(i-j)(i-j+1) / 2}\left[\begin{array}{c}
L+N \\
N-i+j
\end{array}\right]\right)_{1 \leqslant i, j \leqslant \mathscr{P}}
$$

Вычисляя определитель (А.15) с использованием формул Паскаля (А.4), приходим к ответу, выраженному через $q$-биномиальный определитель (см. (142)):

$$
\Sigma_{\mathrm{S}}=q^{N(1-\mathscr{P}) \mathscr{P} / 2}\left(\begin{array}{cccc}
L+N, & L+N+1, & \ldots, & L+N+\mathscr{P}-1 \\
L, & L+1, & \ldots, & L+\mathscr{P}-1
\end{array}\right)_{q} .
$$

Подстановка равенства (А.16) в (А.9) доказывает соотношение (144). Преобразуем определитель в правой части (А.16):

$$
\Sigma_{\mathrm{S}}=q^{N(1-\mathscr{P}) \mathscr{P} / 2} \prod_{j=1}^{L} \prod_{k=1}^{\mathscr{P}} \frac{[N+j+k-1]}{[j+k-1]}\left(\begin{array}{cccc}
N, & N+1, & \ldots, & N+\mathscr{P}-1 \\
0, & 1, & \ldots, & \mathscr{P}-1
\end{array}\right)_{q} .
$$

Стандартное вычисление определителя в (А.17) дает значение $q^{N(\mathscr{P}-1) \mathscr{P} / 2}$. В результате $\Sigma_{S}($ А.17) принимает форму двойного произведения, тем самым доказывается первое соотношение (145).

Двойное произведение в (А.17) совпадает с производящей функцией плоских разбиений в ящике $Z_{q}(L, \mathscr{P}, N)($ см. (1)):

$$
\prod_{j=1}^{L} \prod_{k=1}^{\mathscr{P}} \frac{[N+j+k-1]}{[j+k-1]} \equiv \prod_{j=1}^{L} \prod_{k=1}^{\mathscr{P}} \frac{1-q^{N+j+k-1}}{1-q^{j+k-1}}=Z_{q}(L, \mathscr{P}, N) .
$$

Двойное произведение в левой части (А.18) можно преобразовать к виду

$$
\prod_{j=1}^{L} \prod_{k=1}^{N} \frac{[\mathscr{P}+j+k-1]}{[j+k-1]} \equiv \prod_{j=1}^{L} \prod_{k=1}^{N} \frac{1-q^{\mathscr{P}+j+k-1}}{1-q^{j+k-1}}=Z_{q}(L, N, \mathscr{P}),
$$

откуда следует, что $Z_{q}(L, \mathscr{P}, N)$ совпадает с $Z_{q}(L, N, \mathscr{P})$, и, значит, для $\Sigma_{\mathrm{S}}$ (А.13) имеет место равенство

$$
\Sigma_{\mathrm{S}}=Z_{q}(L, N, \mathscr{P})
$$

Подставляя $\Sigma_{\mathrm{S}}(\mathrm{A.20)}$ в (А.9), получаем второе утверждение, выражаемое (145). Теорема 4 доказана.

Использование (А.7) и (А.8) позволяет представить $q$-параметризованные функции Шура в виде следующих определителей:

$$
\begin{aligned}
S_{\boldsymbol{\lambda}}\left(\frac{\mathbf{q}}{q}\right) & =\operatorname{det}\left(q^{\left(\bar{\lambda}_{i}-i+j\right)\left(\bar{\lambda}_{i}-i+j-1\right) / 2}\left[\begin{array}{l}
N+\mathscr{P}-j \\
\bar{\lambda}_{i}-i+\mathscr{P}
\end{array}\right]\right)_{1 \leqslant i, j \leqslant \mathscr{P}}, \\
S_{\boldsymbol{\lambda}}(\mathbf{q}) & =\operatorname{det}\left(q^{\left(\bar{\lambda}_{i}-i+j\right)\left(\bar{\lambda}_{i}-i+j+1\right) / 2}\left[\begin{array}{l}
N+\mathscr{P}-j \\
\bar{\lambda}_{i}-i+\mathscr{P}
\end{array}\right]\right)_{1 \leqslant i, j \leqslant \mathscr{P}},
\end{aligned}
$$


где используются формулы Паскаля (А.4). Оба представления (А.21) сводятся при $q \rightarrow 1$ к одному предельному выражению $S_{\boldsymbol{\lambda}}(\mathbf{1}) \equiv S_{\boldsymbol{\lambda}}(1,1, \ldots, 1)$ :

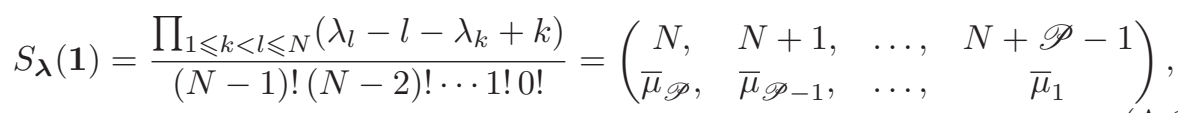

где $\overline{\boldsymbol{\mu}}$ есть строгое сопряженное разбиение. Значение функции Шура в терминах частей разбиения $\boldsymbol{\lambda}$ приведено в [52], и оно дает размерность неприводимого унитарного представления унитарной группы $\mathscr{U}(N)$, отвечающего сигнатуре $\boldsymbol{\lambda}$ [115]. Биномиальный определитель в правой части (А.22) допускает интерпретацию в терминах самоизбегающих решеточных путей, соединяющих точки, координаты которых образованы частями разбиений $(N+\mathscr{P}-1, N+$ $\mathscr{P}-2, \ldots, N)$ и $\left(\bar{\mu}_{1}, \bar{\mu}_{2}, \ldots, \bar{\mu}_{\mathscr{P}}\right)($ см. [108]).

\section{Приложение В. q-бозонная модель и комбинаторика}

В настоящее время интенсивно исследуются так называемые q-бозонные модели [61]-[63], динамические переменные которых связаны с q-бозонными представлениями [116]-[121], естественно возникающими в теории квантовых групп, [9], [122], [123].

Обменная $q$-бозонная модель на одномерной решетке, состоящей из $M+1$ узла, $M+1=0(\bmod 2)$, с периодическими граничными условиями определяется гамильтонианом $\widehat{H}_{\mathrm{q}}$ (см. [119], [120], [124]):

$$
\widehat{H}_{\mathrm{q}}=-\frac{1}{2} \sum_{n \in \mathrm{M}}\left(B_{n}^{\dagger} B_{n+1}+B_{n} B_{n+1}^{\dagger}-2 N_{n}\right) \text {, }
$$

где множество $\mathbf{M}$ определено в п. 2.2. Операторы на узлах $B_{n}, B_{n}^{\dagger}$ и $N_{n}$ образуют $q$-бозонную алгебру:

$$
\left[N_{l}, B_{n}^{\dagger}\right]=B_{l}^{\dagger} \delta_{l n}, \quad\left[N_{l}, B_{n}\right]=-B_{l} \delta_{l n}, \quad\left[B_{l}, B_{n}^{\dagger}\right]=q^{-2 N_{l}} \delta_{l n},
$$

где числовой параметр деформации $q$ можно представить в виде $q=e^{2 \gamma}$. Кажущаяся простота модели (В.1) обманчива, так как в коммутационных соотношениях (В.2) скрыто нелинейное взаимодействие $q$-бозонов.

Для реализации $q$-бозонной алгебры рассмотрим фоковское пространство, натянутое на $N$-частичные векторы состояния

$$
\left|n_{0}, n_{1}, \ldots, n_{M}\right\rangle \equiv \bigotimes_{l=0}^{M}\left|n_{l}\right\rangle_{l}
$$

где $0 \leqslant n_{l} \leqslant N$ и $\sum_{l \in \mathbf{M}} n_{l}=N$. Имеется также вакуумное состояние

$$
|\mathbf{0}\rangle \equiv \bigotimes_{l=0}^{M}|0\rangle_{l}
$$


где $|0\rangle_{l}$ означает такое состояние $|0\rangle$ на $l$-м узле, для которого $B_{l}|0\rangle=0, l \in \mathbf{M}$. Состояния $\left|n_{l}\right\rangle_{l}$ в (В.3) строятся с помощью действия операторов $B_{l}^{\dagger}$ :

$$
\left|n_{l}\right\rangle_{l}=\left(\left[n_{l}\right] !\right)^{-1 / 2}\left(B_{l}^{\dagger}\right)^{n_{l}}|0\rangle_{l},
$$

где $\left[n_{l}\right]$ и $\left[n_{l}\right]$ ! определяются формулами (А.1) и (А.2) соответственно. Представление (В.4) связано с соотношениями

$$
\begin{aligned}
B_{l}^{\dagger}\left|n_{l}\right\rangle_{l} & =\left[n_{l}+1\right]^{1 / 2}\left|n_{l}+1\right\rangle_{l}, \\
B_{l}\left|n_{l}\right\rangle_{l} & =\left[n_{l}\right]^{1 / 2}\left|n_{l}-1\right\rangle_{l}, \\
\widehat{N}_{l}\left|n_{l}\right\rangle_{l} & =n_{l}\left|n_{l}\right\rangle_{l} .
\end{aligned}
$$

Состояния (В.3) ортогональны:

$$
\left\langle p_{0}, p_{1}, \ldots, p_{M} \mid n_{0}, n_{1}, \ldots, n_{M}\right\rangle=\delta_{p_{0} n_{0}} \delta_{p_{1} n_{1}} \cdots \delta_{p_{M} n_{M}} .
$$

Операторы (В.2) могут быть выражены через канонические бозонные операторы $b_{l}^{\dagger}, b_{l}$, которые подчиняются перестановочному соотношению $\left[b_{n}, b_{l}^{\dagger}\right]=\delta_{n l}$ :

$$
B_{l}=\left(B_{l}^{\dagger}\right)^{\dagger}=\sqrt{\frac{\left[N_{l}+1\right]}{N_{l}+1}} b_{l}, \quad N_{l}=b_{l}^{\dagger} b_{l} .
$$

При $q \rightarrow 1(\gamma \rightarrow 0)$-бозоны становятся обычными бозонами, $B_{l} \rightarrow b_{l}, B_{l}^{\dagger} \rightarrow b_{l}^{\dagger}$, a $q$-бозонная модель сводится к линейной бозонной обменной модели с гамильтонианом

$$
\widehat{H}_{\mathrm{b}}=-\frac{1}{2} \sum_{n \in \mathbf{M}}\left(b_{n}^{\dagger} b_{n+1}+b_{n} b_{n+1}^{\dagger}-2 N_{n}\right)
$$

В пределе сильной связи $q \rightarrow \infty(\gamma \rightarrow \infty)$-бозонные операторы преобразуются в операторы $\phi_{l}, \phi_{l}^{\dagger}, N_{l}$ с перестановочными соотношениями

$$
\left[N_{n}, \phi_{l}\right]=-\phi_{n} \delta_{n l}, \quad\left[N_{n}, \phi_{l}^{\dagger}\right]=\phi_{n}^{\dagger} \delta_{n l}, \quad\left[\phi_{n}, \phi_{l}^{\dagger}\right]=\pi_{n} \delta_{n l},
$$

где $\pi_{n}$ является вакуумным проектором: $\pi_{n}=(|0\rangle\langle 0|)_{n}$. В этом пределе $q$-бозонный гамильтониан принимает вид

$$
\widehat{H}_{\mathrm{ph}}=-\frac{1}{2} \sum_{n \in \mathbf{M}}\left(\phi_{n}^{\dagger} \phi_{n+1}+\phi_{n} \phi_{n+1}^{\dagger}-2 N_{n}\right)
$$

и называется гамильтонианом фазовой модели. Введенные операторы $\phi_{l}$ являются изометрическими и удовлетворяют соотношениям $\phi_{l} \phi_{l}^{\dagger}=1, \phi_{l}^{\dagger} \phi_{l}=1-\pi_{l}$. Операторы экспоненциальной фазы $\phi_{l}, \phi_{l}^{\dagger}$ (см. [125]) могут быть представлены через бозонные операторы:

$$
\phi_{l}=\left(N_{l}+1\right)^{-1 / 2} b_{l}, \quad \phi_{l}^{\dagger}=b_{l}^{\dagger}\left(N_{l}+1\right)^{-1 / 2}, \quad N_{l}=b_{l}^{\dagger} b_{l} .
$$

Переход к непрерывному пределу осуществляется соотношениями $n \delta \rightarrow x$, $M \delta \rightarrow L$, где постоянная решетки $\delta$ стремится к нулю, $\delta \rightarrow 0$. При этом 
$q$-бозонный гамильтониан приводит к гамильтониану системы бозе-полей $\psi^{\dagger}(x)$, $\psi(x)$ на отрезке $[0, L] \subset \mathbb{R}:$

$$
\widehat{H}=\int_{0}^{L}\left(\psi^{\dagger}(x)\left(-\frac{\partial^{2}}{\partial x^{2}}\right) \psi(x)+\frac{g}{2} \psi^{\dagger}(x) \psi^{\dagger}(x) \psi(x) \psi(x)\right) d x,
$$

а поля $\psi^{\dagger}(x), \psi(x)$ подчиняются каноническому перестановочному соотношению $\left[\psi(x), \psi^{\dagger}(y)\right]=\delta(x-y)$.

КМОЗ приводит к собственным векторам и собственным числам $q$-бозонной модели (В.1) (см. [119], [120], [124]), а соответствующий $L$-оператор модели

$$
L_{\mathrm{q}}(n \mid u)=\left(\begin{array}{cc}
u^{-1} & \chi B_{n}^{\dagger} \\
\chi B_{n} & u
\end{array}\right)
$$

где $u \in \mathbb{C}, \chi=\left(1-q^{-2}\right)^{1 / 2}$, удовлетворяет сплетающему соотношению с $R$-матрицей (16) при $h=\gamma$. Гамильтониан выражается через соответствующую матрицу монодромии (24):

$$
-2 \chi \widehat{H}_{\mathrm{q}}=\left.\frac{\partial u^{M+1} \tau(u)}{\partial u^{2}}\right|_{u=0}+\left.\frac{\partial u^{-M-1} \tau(u)}{\partial u^{-2}}\right|_{u=\infty}-2 \chi^{2} \widehat{N},
$$

где оператор числа частиц $\widehat{N}=\sum_{n \in \mathbf{M}} N_{n}$ перестановочен с гамильтонианом. Векторы состояния для $N$ частиц (и соответствующие сопряженные векторы) строятся из вакуумного вектора $|\mathbf{0}\rangle$ (см. (В.3)):

$$
\left|\Psi_{N}(\mathbf{u})\right\rangle=\prod_{j=1}^{N} u_{j}^{M} B\left(u_{j}\right)|\mathbf{0}\rangle, \quad\left\langle\Psi_{N}(\mathbf{u})\right|=\langle\mathbf{0}| \prod_{j=1}^{N} u_{j}^{-M} C\left(u_{j}\right),
$$

где набор параметров $\mathbf{u}$ определен так же, как в (33). Отметим, что волновые функции состояний (В.9) выражаются через функции Холла-Литтлвуда [61]. Уравнения Бете $q$-бозонной модели (В.1) имеют вид

$$
u_{i}^{-2(M+1)}=(-1)^{N-1} \prod_{j=1}^{N} \frac{u_{i}^{2} q-u_{j}^{2} q^{-1}}{u_{j}^{2} q-u_{i}^{2} q^{-1}}, \quad 1 \leqslant i \leqslant N .
$$

Собственные энергии гамильтониана (В.1) суть

$$
E_{N}=\sum_{k=1}^{N}\left(1-\frac{u_{k}^{2}+u_{k}^{-2}}{2}\right)
$$

Пусть $N$ частиц заполняют узлы периодической цепочки длины $M+1$. Число частиц на каждом узле произвольно, и любая конфигурация частиц представлена последовательностью чисел заполнения $\left(n_{M}, \ldots, n_{1}, n_{0}\right), \sum_{l \in \mathbf{M}} n_{l}=N$. Каждая частица перескакивает с вероятностью $1 / 2$ на один из ближайших узлов, и конфигурации частиц для обменного процесса описываются гамильтонианом (В.7) и векторами Фока (В.3), для которых $\left|n_{l}\right\rangle_{l}=\left(\phi_{l}^{\dagger}\right)^{n_{l}}|0\rangle_{l}$. При этом представление алгебры (В.6) в пространстве векторов (В.3) возникает из (В.5):

$$
\phi_{l}^{\dagger}\left|n_{l}\right\rangle_{l}=\left|n_{l}+1\right\rangle_{l}, \quad \phi_{l}\left|n_{l}\right\rangle_{l}=\left|n_{l}-1\right\rangle_{l}, \quad \widehat{N}_{l}\left|n_{l}\right\rangle_{l}=n_{l}\left|n_{l}\right\rangle_{l} .
$$


Вакуумный вектор есть $|\mathbf{0}\rangle \equiv \bigotimes_{l=0}^{M}|0\rangle_{l}$, где $|0\rangle_{l}$ - состояние $|0\rangle$ на $l$-м узле и $\phi_{l}|0\rangle=0, l \in \mathbf{M}$.

Для фазовой модели $L$-оператор имеет следующий вид [119]-[121], [124]:

$$
L_{\mathrm{ph}}(n \mid u)=\left(\begin{array}{cc}
u^{-1} & \phi_{n}^{\dagger} \\
\phi_{n} & u
\end{array}\right)
$$

где $u \in \mathbb{C}$, и удовлетворяет сплетающему соотношению с $R$-матрицей (18). Гамильтониан фазовой модели (В.7) выражается через матрицу перехода:

$$
-2 \widehat{H}_{\mathrm{ph}}=\left.\frac{\partial u^{M+1} \tau(u)}{\partial u^{2}}\right|_{u=0}+\left.\frac{\partial u^{-M-1} \tau(u)}{\partial u^{-2}}\right|_{u=\infty}-2 \widehat{N} .
$$

Векторы состояния (В.9), в которых операторы $B(u)$ и $C(u)$ строятся с помощью $L$-оператора (В.13), являются собственными для гамильтониана (B.7) тогда и только тогда, когда элементы и удовлетворяют уравнениям Бете

$$
u_{i}^{-2(M+N+1)}=(-1)^{N-1} \prod_{j=1}^{N} u_{j}^{-2}, \quad 1 \leqslant i \leqslant N .
$$

Собственные значения гамильтониана (В.7) выражаются через решения этих уравнений по формуле (В.11).

Используя явные выражения для $B(u)$ и $C(u)$, можно представить вектор состояния (В.9) в "координатном" виде [62]:

$$
\left|\Psi_{N}(\mathbf{u})\right\rangle=\sum_{\boldsymbol{\lambda} \subseteq\left\{M^{N}\right\}} S_{\boldsymbol{\lambda}}\left(\mathbf{u}^{2}\right)\left(\prod_{l=0}^{M}\left(\phi_{l}^{\dagger}\right)^{n_{l}}\right)|\mathbf{0}\rangle
$$

где $S_{\boldsymbol{\lambda}}\left(\mathbf{u}^{2}\right)$ - функция Шура (35) и суммирование идет по нестрогим разбиениям $\boldsymbol{\lambda}$ длины $l(\boldsymbol{\lambda})=N, N \leqslant M$. Существует взаимно однозначное соответствие между конфигурацией чисел заполнения $\left(n_{M}, \ldots, n_{1}, n_{0}\right), \sum_{l \in \mathbf{M}} n_{l}=N$, и разбиением $\boldsymbol{\lambda}=\left(M^{n_{M}}, \ldots, 1^{n_{1}}, 0^{n_{0}}\right)$, в котором $S^{n_{S}}$ означает, что номер узла $S$ повторяется $n_{S}$ раз. Соответствие между конфигурациями частиц и частями разбиения $\boldsymbol{\lambda}$ представлено на рис. 15 .

Вектор состояния, сопряженный (В.15), имеет вид

$$
\left\langle\Psi_{N}(\mathbf{v})\right|=\sum_{\boldsymbol{\lambda} \subseteq\left\{M^{N}\right\}} S_{\boldsymbol{\lambda}}\left(\mathbf{v}^{-2}\right)\langle\mathbf{0}|\left(\prod_{l=0}^{M}\left(\phi_{l}\right)^{n_{l}}\right) .
$$

Скалярное произведение $N$-частичных векторов состояния для произвольных $\mathbf{u}$ и $\mathbf{v}$ дается формулой Бине-Коши (78):

$$
\begin{gathered}
\left\langle\Psi_{N}(\mathbf{v}) \mid \Psi_{N}(\mathbf{u})\right\rangle=\sum_{\boldsymbol{\lambda} \subseteq\left\{M^{N}\right\}} S_{\boldsymbol{\lambda}}\left(\mathbf{v}^{-2}\right) S_{\boldsymbol{\lambda}}\left(\mathbf{u}^{2}\right)=\frac{\operatorname{det}\left(T_{k j}\right)_{1 \leqslant k, j \leqslant N}}{\mathscr{V}_{N}\left(\mathbf{u}^{2}\right) \mathscr{V}_{N}\left(\mathbf{v}^{-2}\right)} \\
T_{k j}=h_{M+N}\left(u_{k}^{2} v_{j}^{-2}\right)
\end{gathered}
$$




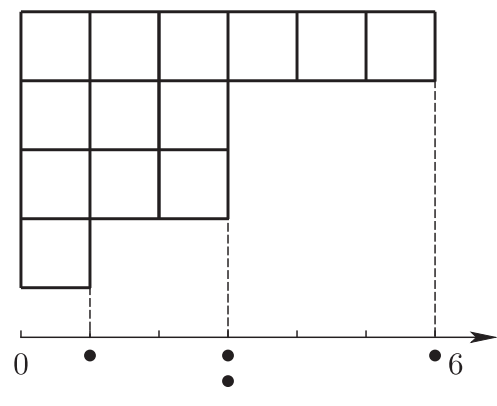

Рис. 15. Конфигурация частиц $(N=4)$ на решетке $(M=6)$ и диаграмма Юнга соответствующего разбиения

где функция $h_{P}(x)$ определена в (47). Скалярное произведение (В.16) в q-параметризации (42) принимает вид

$$
\left\langle\Psi_{N}\left(\mathbf{q}^{-1 / 2}\right) \mid \Psi_{N}\left(\left(\frac{\mathbf{q}}{q}\right)^{1 / 2}\right)\right\rangle=\frac{\operatorname{det}\left(h_{M+N}\left(q^{j+k-1}\right)\right)_{1 \leqslant j, k \leqslant N}}{\mathscr{V}_{N}(\mathbf{q})_{N}(\mathbf{q} / q)}
$$

Сопоставляя (В.17) и (162) при $n=0$, получаем с учетом (5), что скалярное произведение $\left\langle\Psi_{N}\left(\mathbf{q}^{-1 / 2}\right) \mid \Psi_{N}\left((\mathbf{q} / q)^{1 / 2}\right)\right\rangle$ для фазовой модели равно производящей функции $Z_{q}(N, N, M)$ плоских разбиений в $\mathscr{B}(N, N, M)$.

Двумерная классическая вершинная модель, связанная с фазовой моделью, может быть определена следующим образом [62], [63]. Матричные элементы $L$-оператора $L_{\mathrm{ph}}(n \mid u)($ В.13), как и в случае $L$-оператора четырехвершинной модели (17), соответствуют вершинам с примыкающими стрелками: вершине (iii) (рис. 4) отвечает матричный элемент $\left(L_{\mathrm{ph}}\right)_{12}=\phi_{n}^{\dagger}$, вершине (iv) отвечает элемент $\left(L_{\mathrm{ph}}\right)_{21}=\phi_{n}$, а вершинам (i) и (ii) отвечают элементы $\left(L_{\mathrm{ph}}\right)_{11}=u^{-1}$ и $\left(L_{\mathrm{ph}}\right)_{22}=u$ соответственно. Матричные элементы матрицы монодромии $(20)$ выражаются через суммы по всем возможным конфигурациям стрелок на цепочке $M+1$ узлов (рис. 5).

Пусть решетка имеет размер $2 N \times(M+1)$. Операторы $A\left(u_{j}\right), B\left(u_{j}\right), C\left(u_{j}\right)$, $D\left(u_{j}\right)$ ассоциируются с $j$-й вертикальной линией решетки. Каждой $l$-й горизонтальной линии соответствует локальный фоковский вектор $\left|n_{l}\right\rangle_{l}$, где $n_{l}$ - число заполнения. Статистическая сумма модели на решетке с заданными граничными условиями равна сумме всех допустимых конфигураций, определяемых вершинами (i), (ii), (iii), (iv) (рис. 4) и возможными числами заполнения.

Скалярное произведение (В.16) равно статистической сумме введенной вершинной модели с граничными условиями: внешние стрелки на первых $N$ столбцах (ассоциируемых с операторами $\left.C\left(v_{i}\right)\right)$ направлены внутрь, а на $N$ последних (ассоциируемых с операторами $B\left(u_{j}\right)$ ) наружу; на правой и на левой границах все числа заполнения равны нулю.

Допустимые конфигурации отвечают наборам из $N$ самоизбегающих путей, начинающихся в узлах $(-N+i-1 ; 0)$ и заканчивающихся в узлах $(i ; M)$, $1 \leqslant i \leqslant N$ (см. рис. 16). Два различных пути не проходят по одному и тому же вертикальному ребру решетки, между тем любое их число может проходить по горизонтальному ребру. Число путей, проходящих по одному и тому 


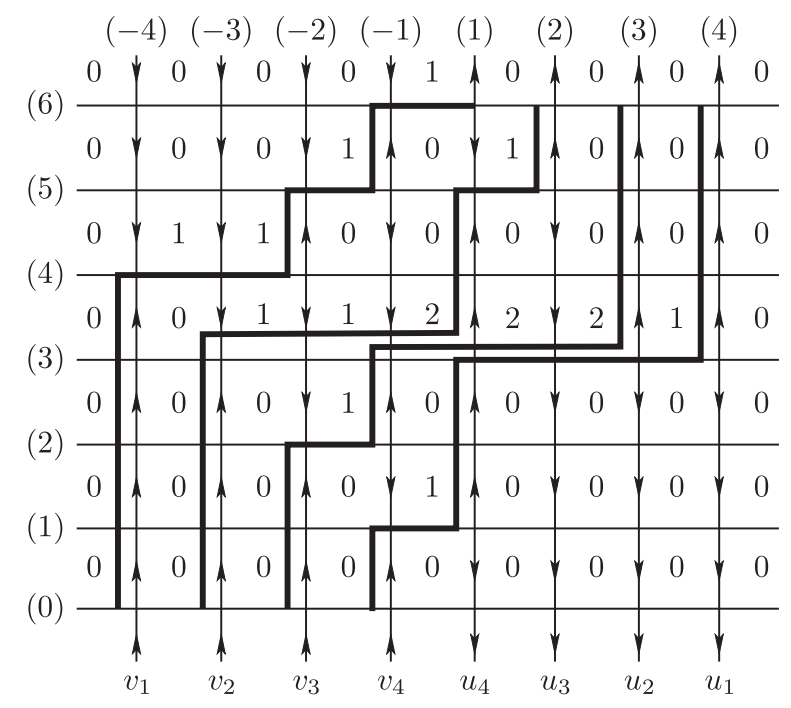

Рис. 16. Типичный набор решеточных путей для фазовой модели

же горизонтальному ребру, равно числу заполнения этого ребра (рис. 16). Так как лишь один путь допустим на вертикальных ребрах, то полученная конфигурация путей биективна конфигурации типа "арбуз" на квадратной решетке из $2 N \times(M+N)$ узлов и может быть представлена как плоское разбиение в ящике $\mathscr{B}(N, N, M)$.

Скалярное произведение (В.16) принимает вид

$$
\left\langle\Psi_{N}(\mathbf{v}) \mid \Psi_{N}(\mathbf{u})\right\rangle=\sum \prod_{k=-1}^{-N} v_{-k}^{l_{k}^{(2)}-l_{k}^{(1)}-M} \prod_{j=1}^{N} u_{j}^{l_{j}^{(2)}-l_{j}^{(1)}+M},
$$

где суммирование идет по всем допустимым путям, а $l_{k}^{(1)}$ и $l_{k}^{(2)}$ - числа вершин типов (i) и (ii) (рис. 4) в $k$-м столбце. Объем плоского разбиения $\boldsymbol{\pi}$ в ящике $\mathscr{B}(N, N, M)$ вычисляется по следующей формуле [62]:

$$
|\boldsymbol{\pi}|=\frac{1}{2} \sum_{k=-1}^{-N} k\left(l_{k}^{(2)}-l_{k}^{(1)}-M\right)+\frac{1}{2} \sum_{j=1}^{N}(j-1)\left(l_{j}^{(2)}-l_{j}^{(1)}+M\right) .
$$

Использование в (В.18) параметризации (42) приводит к ответу для скалярного произведения (В.17) в виде производящей функции (1).

\section{Список литературы}

[1] Л. Д. Фаддеев, Л. А. Тахтаджян, "Квантовый метод обратной задачи и $X Y Z$ модель Гейзенберга", УМH, 34:5(209) (1979), 13-63; англ. пер.: L. A. Takhtadzhyan, L. D. Faddeev, "The quantum method of the inverse problem and the Heisenberg XYZ model", Russian Math. Surveys, 34:5 (1979), 11-68. 
[2] L. D. Faddeev, "Quantum completely integrable models in field theory", Sov. Sci. Rev. C: Math. Phys., 1 (1980), 107-160; 40 years in mathematical physics, World Sci. Ser. 20th Century Math., 2, World Sci. Publ., River Edge, NJ, 1995, 187-235.

[3] Е.К. Склянин, Л.А. Тахтаджян, Л.Д. Фаддеев, "Квантовый метод обратной задачи. I", ТМФ, 40:2 (1979), 194-220; англ. пер.: E. K. Sklyanin, L. A. Takhtadzhyan, L. D. Faddeev, "Quantum inverse problem method. I", Theoret. and Math. Phys., 40:2 (1979), 688-706.

[4] P. P. Kulish, E. K. Sklyanin, "Quantum spectral transform method. Recent developments", Integrable quantum field theories (Tvärminne, 1981), Lecture Notes in Phys., 151, Springer, Berlin-New York, 1982, 61-119.

[5] P.P. Kulish, F.A. Smirnov, "Anisotropic Heisenberg ferromagnet with a ground state of the domain wall type", J. Phys. C, 18:5 (1985), 1037-1048.

[6] L. D. Faddeev, "How the algebraic Bethe ansatz works for integrable models", Symétries quantiques (Les Houches, 1995), North Holland, Amsterdam, 1998, 149-219.

[7] Л.Д. Фаддеев, "Новая жизнь полной интегрируемости”, УФН, 183:5 (2013), 487-495; англ. пер.: L. D. Faddeev, "The new life of complete integrability", Phys. Usp., 56:5 (2013), 465-472.

[8] H. Bethe, "Zur Theorie der Metalle. I. Eigenwerte und Eigenfunktionen der linearen Atomkette", Z. Phys., 71:3-4 (1931), 205-226.

[9] Н. Ю. Решетихин, Л. А. Тахтаджян, Л. Д. Фаддеев, "Квантование групп Ли и алгебр Ли", Алгебра и анализ, 1:1 (1989), 178-206; англ. пер.: N. Yu. Reshetikhin, L. A. Takhtadzhyan, L. D. Faddeev, "Quantization of Lie groups and Lie algebras", Leningrad Math. J., 1:1 (1990), 193-225.

[10] Н. Ю. Решетихин, "Квазитреугольные алгебры Хопфа и инварианты связок", Алгебра и анализ, 1:2 (1989), 169-188; англ. пер.: N. Yu. Reshetikhin, "Quasitriangular Hopf algebras and invariants of links", Leningrad Math. J., 1:2 (1990), 491-513.

[11] V. V. Bazhanov, S. L. Lukyanov, A. B. Zamolodchikov, "Integrable structure of conformal field theory, quantum KdV theory and thermodynamic Bethe ansatz", Comm. Math. Phys., 177:2 (1996), 381-398.

[12] L. D. Faddeev, A. Yu. Volkov, "Discrete evolution for the zero modes of the quantum Liouville model", J. Phys. A, 41:19 (2008), 194008, 12 pp.

[13] A. Bytsko, J. Teschner, "The integrable structure of nonrational conformal field theory", Adv. Theor. Math. Phys., 17:4 (2013), 701-740.

[14] N. A. Nekrasov, S. L. Shatashvili, "Supersymmetric vacua and Bethe ansatz", Nuclear Phys. B Proc. Suppl., 192/193 (2009), 91-112.

[15] N. Nekrasov, S. Shatashvili, "Quantum integrability and supersymmetric vacua", Prog. Theor. Phys. Suppl., 177 (2009), 105-119.

[16] А. Ю. Волков, Л. Д. Фаддеев, "Янг-бакстеризация квантового дилогарифма", Вопросы квантовой теории поля и статистической физики. 13, Зап. науч. сем. ПОМИ, 224, ПОМИ, СПб., 1995, 146-154; англ. пер.: А. Yu. Volkov, L. D. Faddeev, "Yang-baxterization of the quantum dilogarithm", J. Math. Sci. (N. Y.), 88:2 (1998), 202-207.

[17] V. Tarasov, A. Varchenko, "Combinatorial formulae for nested Bethe vectors", SIGMA, 9 (2013), 048, 28 pp.

[18] С.Э. Деркачев, В.П. Спиридонов, "Уравнение Янга-Бакстера, перестановки параметров и эллиптический бета-интеграл", УМН, 68:6(414) (2013), 59-106; 
англ. пер.: S. ̇̀. Derkachev, V.P. Spiridonov, "Yang-Baxter equation, parameter permutations, and the elliptic beta integral", Russian Math. Surveys, 68:6 (2013), 1027-1072.

[19] R. P. Stanley, Enumerative combinatorics, v. 1, Cambridge Stud. Adv. Math., 49, Cambridge Univ. Press, Cambridge, 1997, xi+325 pp.; v. 2, Cambridge Stud. Adv. Math., 62, Cambridge Univ. Press, Cambridge, 1999, xii+581 pp.

[20] G. E. Andrews, The theory of partitions, Cambridge Math. Lib., Cambridge Univ. Press, Cambridge, 1998, xvi+255 pp.

[21] Р. Бэкстер, Точно решаемые модели в статистической механике, Мир, М., 1985, 488 с.; пер. с англ.: R. J. Baxter, Exactly solved models in statistical mechanics, Academic Press, Inc., London, 1982, xii+486 pp.

[22] E. H. Lieb, F. Y. Wu, "Two dimensional ferroelectric models", Phase transitions and critical phenomena, v. 1, Academic Press, London, 1972, 331-490.

[23] М. Годен, Волновая функиия Бете, Мир, М., 1987, 352 с.; пер. с франц.: M. Gaudin, La fonction d'onde de Bethe, Collect. Commissariat Energ. Atom. Ser. Sci., Masson, Paris, 1983, xvi+331 pp.

[24] V. E. Korepin, "Calculation of norms of Bethe wave functions", Comm. Math. Phys., 86:3 (1982), 391-418.

[25] А. Г. Изергин, "Статсумма шестивершинной модели в конечном объеме", Докл. AH CCCP, 297:2 (1987), 331-333; англ. пер.: A. G. Izergin, "Partition function of a six-vertex model in a finite volume", Soviet Phys. Dokl., 32:11 (1987), 878-879.

[26] V. Korepin, P. Zinn-Justin, "Thermodynamic limit of the six-vertex model with domain wall boundary conditions", J. Phys. A, 33:40 (2000), 7053-7066.

[27] N. M. Bogoliubov, A. G. Pronko, M. B. Zvonarev, "Boundary correlation functions of the six-vertex model", J. Phys. A, 35:27 (2002), 5525-5541.

[28] G. Kuperberg, "Another proof of the alternating-sign matrix conjecture", Int. Math. Res. Not., 1996:3 (1996), 139-150.

[29] W. H. Mills, D. P. Robbins, H. Rumsey, Jr., "Alternating sign matrices and descending plane partitions", J. Combin. Theory Ser. A, 34:3 (1983), 340-359.

[30] D. M. Bressoud, Proofs and confirmations. The story of the alternating sign matrix conjecture, MAA Spectrum, Math. Assoc. America, Washington, DC; Cambridge Univ. Press, Cambridge, 1999, xvi+274 pp.

[31] N. Elkies, G. Kuperberg, M. Larsen, J. Propp, "Alternating-sign matrices and domino tilings. I", J. Algebraic Combin., 1:2 (1992), 111-132.

[32] F. Colomo, A. G. Pronko, "Square ice, alternating sign matrices, and classical orthogonal polynomials", J. Stat. Mech. Theory Exp., 2005, № 1, P01005, 33 pp. (electronic).

[33] D. Betea, M. Wheeler, P. Zinn-Justin, "Refined Cauchy/Littlewood identities and six-vertex model partition functions. II. Proofs and new conjectures", J. Algebraic Combin., 42:2 (2015), 555-603; 2014, 34 pp., arXiv: 1405.7035.

[34] P. Zinn-Justin, Six-vertex, loop and tiling models: integrability and combinatorics, 2009, 77 pp., arXiv: 0901.0665.

[35] Н. М. Боголюбов, "Четырехвершинная модель и случайные укладки", ТМФ, 155:1 (2008), 25-38; англ. пер.: N. M. Bogoliubov, "Four-vertex model and random tilings", Theoret. and Math. Phys., 155:1 (2008), 523-535.

[36] D. Allison, N. Reshetikhin, "Numerical study of the 6-vertex model with domain wall boundary conditions", Ann. Inst. Fourier (Grenoble), 55:6 (2005), 1847-1869. 
[37] F. Colomo, V. Noferini, A. G. Pronko, "Algebraic arctic curves in the domain-wall six-vertex model", J. Phys. A, 44:19 (2011), 195201, 13 pp.

[38] F. Colomo, A. G. Pronko, P. Zinn-Justin, "The arctic curve of the domain-wall six-vertex model in its antiferroelectric regime", J. Stat. Mech. Theory Exp., 2010, № 3, L03002, 11 pp.

[39] А. М. Вершик, С.В. Керов, "Асимптотика мер Планшереля симметрических групп и предельная форма диаграмм Юнга", Докл. АН СCCP, 233:6 (1977), 1024-1027; англ. пер.: А. M. Vershik, S. V. Kerov, "Asymptotics of the Plancherel measure of the symmetric group and the limiting form of Young tableaux", Soviet Math. Dokl., 18 (1977), 527-531.

[40] А. М. Вершик, "Статистическая механика комбинаторных разбиений и их предельные конфигурации", Функи. анализ и его прил., 30:2 (1996), 19-39; англ. пер.: A.M. Vershik, "Statistical mechanics of combinatorial partitions, and their limit shapes", Funct. Anal. Appl., 30:2 (1996), 90-105.

[41] A. Okounkov, "Infinite wedge and random partitions", Selecta Math. (N. S.), 7:1 (2001), 57-81.

[42] A. Okounkov, N. Reshetikhin, "Random skew plane partitions and the Pearcey process", Comm. Math. Phys., 269:3 (2007), 571-609.

[43] P. A. MacMahon, Combinatory analysis, v. 1, 2, Cambridge Univ. Press, Cambridge, 1915, 1916, xx+300 pp., xix+340 pp.

[44] M.E. Fisher, "Walks, walls, wetting, and melting", J. Statist. Phys., 34:5-6 (1984), $667-729$.

[45] S. Redner, A guide to first-passage processes, Cambridge Univ. Press, Cambridge, 2001, $\mathrm{x}+312 \mathrm{pp}$.

[46] P. J. Forrester, "Exact solution of the lock step model of vicious walkers", J. Phys. A, 23:7 (1990), 1259-1273.

[47] T. Nagao, P. J. Forrester, "Vicious random walkers and a discretization of Gaussian random matrix ensembles", Nuclear Phys. B, 620:3 (2002), 551-565.

[48] A. J. Guttmann, A.L. Owczarek, X.G. Viennot, "Vicious walkers and Young tableaux. I. Without walls", J. Phys. A, 31:40 (1998), 8123-8135.

[49] C. Krattenthaler, A. J. Guttmann, X. G. Viennot, "Vicious walkers, friendly walkers and Young tableaux. II. With a wall", J. Phys. A, 33:48 (2000), 8835-8866.

[50] C. Krattenthaler, A. J. Guttmann, X. G. Viennot, "Vicious walkers, friendly walkers, and Young tableaux. III. Between two walls", J. Statist. Phys., 110:3-6 (2003), 1069-1086.

[51] A. Borodin, G. Olshanski, "Infinite-dimensional diffusions as limits of random walks on partitions", Probab. Theory Related Fields, 144:1-2 (2009), 281-318.

[52] I. G. Macdonald, Symmetric functions and Hall polynomials, 2nd ed., Oxford Math. Monogr., Oxford Univ. Press, New York, 1995, x+475 pp.; рус. пер. 1-го изд.: И. Макдональд, Симметрические функиии и многочлены Холла, Мир, М., 1984, $224 \mathrm{c}$.

[53] R. Rajesh, D. Dhar, "An exactly solvable anisotropic directed percolation model in three dimensions", Phys. Rev. Lett., 81:8 (1998), 1646-1649.

[54] A. Okounkov, N. Reshetikhin, "Correlation function of Schur process with application to local geometry of a random 3-dimensional Young diagram", J. Amer. Math. Soc., 16:3 (2003), 581-603 (electronic).

[55] P. L. Ferrari, H. Spohn, "Step fluctuations for a faceted crystal", J. Statist. Phys., 113:1-2 (2003), 1-46. 
[56] T. Nakatsu, K. Takasaki, "Melting crystal, quantum torus and Toda hierarchy", Comm. Math. Phys., 285:2 (2009), 445-468.

[57] A. Okounkov, N. Reshetikhin, C. Vafa, "Quantum Calabi-Yau and classical crystals", The unity of mathematics, In honor of the ninetieth birthday of I. M. Gelfand, Progr. Math., 244, Birkhäuser Boston, Boston, MA, 2006, 597-618.

[58] Н. М. Боголюбов, “XX0 Цепочка Гейзенберга и случайные блуждания”, Teория представлений, динамические системы, комбинаторные и алгоритмические методы. ХІІ, Зап. науч. сем. ПОМИ, 325, ПОМИ, СПб., 2005, 13-27; англ. пер.: N. M. Bogoliubov, "XX0 Heisenberg chain and random walks", J. Math. Sci. (N. Y.), 138:3 (2006), 5636-5643.

[59] Н. М. Боголюбов, "Интегрируемые модели для зловредных и дружественных пешеходов", Вопросы квантовой теории поля и статистической физики. 19, Зап. науч. сем. ПОМИ, 335, ПОМИ, СПб., 2006, 59-74; англ. пер.: N. M. Bogoliubov, "Integrable models for the vicious and friendly walkers", J. Math. Sci. (N. Y.), 143:1 (2007), 2729-2737.

[60] Н. М. Боголюбов, К. Л. Малышев, "Корреляционные функции $X X$-магнетика Гейзенберга и случайные блуждания недружественных пешеходов", ТМФ, 159:2 (2009), 179-193; англ. пер.: N. M. Bogoliubov, C. Malyshev, "Correlation functions of the XX Heisenberg magnet and random walks of vicious walkers", Theoret. and Math. Phys., 159:2 (2009), 563-574.

[61] Н. В. Цилевич, "Квантовый метод обратной задачи для $q$-бозонной модели и симметрические функции”, Функи. анализ и его прил., 40:3 (2006), 53-65; англ. пер.: N.V. Tsilevich, "Quantum inverse scattering method for the $q$-boson model and symmetric functions", Funct. Anal. Appl., 40:3 (2006), 207-217.

[62] N. M. Bogoliubov, "Boxed plane partitions as an exactly solvable boson model", J. Phys. A, 38:43 (2005), 9415-9430.

[63] Н. М. Боголюбов, "Перечисление плоских разбиений и алгебраический анзац Бете", ТМФ, 150:2 (2007), 193-203; англ. пер.: N. M. Bogolyubov, "Enumeration of plane partitions and the algebraic Bethe anzatz", Theoret. and Math. Phys., 150:2 (2007), 165-174.

[64] В. Гейзенберг, "К теории ферромагнетизма", Избранные труды, Эдиториал УPCC, M., 2001, 382-395; пер. с нем.: W. Heisenberg, "Zur Theorie des Ferromagnetismus", Z. Phys., 49:9-10 (1928), 619-636.

[65] C. N. Yang, C. P. Yang, "One-dimensional chain of anisotropic spin-spin interactions. I. Proof of Bethe's hypothesis for ground state in a finite system", Phys. Rev., 150:1 (1966), 321-327.

[66] C. N. Yang, C. P. Yang, "One-dimensional chain of anisotropic spin-spin interactions. II. Properties of the ground-state energy per lattice site for an infinite system", Phys. Rev., 150:1 (1966), 327-339.

[67] C. N. Yang, C. P. Yang, "One-dimensional chain of anisotropic spin-spin interactions. III. Applications", Phys. Rev., 151:1 (1966), 258-264.

[68] A. V. Razumov, Yu. G. Stroganov, "Spin chains and combinatorics", J. Phys. A, 34:14 (2001), 3185-3190.

[69] P. Deift, Orthogonal polynomials and random matrices: a Riemann-Hilbert approach, Courant Lect. Notes Math., 3, New York Univ., Courant Inst. Math. Sci., New York, NY; Amer. Math. Soc., Providence, RI, 1999, viii+273 pp.

[70] Н. М. Боголюбов, А. Г. Изергин, В.Е. Корепин, Корреляиионные функции интегрируемых систем и квантовый метод обратной задачи, Наука, М., 1992, $240 \mathrm{c.}$ 
[71] V.E. Korepin, N. M. Bogoliubov, A. G. Izergin, Quantum inverse scattering method and correlation functions, Cambridge Monogr. Math. Phys., Cambridge Univ. Press, Cambridge, 1993, xx+555 pp.

[72] A. G. Izergin, V.E. Korepin, "Correlation functions for the Heisenberg $X X Z$-antiferromagnet", Comm. Math. Phys., 99:2 (1985), 271-302.

[73] F. H. L. Eßler, H. Frahm, A. G. Izergin, V.E. Korepin, "Determinant representation for correlation functions of spin-1/2 XXX and XXZ Heisenberg magnets", Comm. Math. Phys., 174:1 (1995), 191-214.

[74] N. Kitanine, J. M. Maillet, V. Terras, "Form factors of the XXZ Heisenberg spin- $\frac{1}{2}$ finite chain", Nuclear Phys. B, 554:3 (1999), 647-678.

[75] N. Kitanine, J. M. Maillet, N. A. Slavnov, V. Terras, "Correlation functions of the $X X Z$ spin- $\frac{1}{2}$ Heisenberg chain at the free fermion point from their multiple integral representations", Nuclear Phys. B, 642:3 (2002), 433-455.

[76] N. Kitanine, J. M. Maillet, N.A. Slavnov, V. Terras, "Large distance asymptotic behaviour of the emptiness formation probability of the $X X Z$ spin- $\frac{1}{2}$ Heisenberg chain", J. Phys. A, 35:49 (2002), L753-L758.

[77] Н. А. Славнов, "Алгебраический анзац Бете и квантовые интегрируемые системы", УМH, 62:4(376) (2007), 91-132; англ. пер.: N. A. Slavnov, "The algebraic Bethe ansatz and quantum integrable systems", Russian Math. Surveys, 62:4 (2007), $727-766$.

[78] N. Kitanine, J. M. Maillet, N. A. Slavnov, V. Terras, "Spin-spin correlation functions of the $X X Z-\frac{1}{2}$ Heisenberg chain in a magnetic field", Nuclear Phys. B, 641:3 (2002), $487-518$.

[79] L. Carlitz, "Some determinants of q-binomial coefficients", J. Reine Angew. Math., 1967:226 (1967), 216-220.

[80] N. M. Bogoliubov, C. Malyshev, "Correlation functions of XX0 Heisenberg chain, q-binomial determinants, and random walks", Nuclear Phys. B, 879 (2014), 268-291.

[81] P. J. Forrester, S. O. Warnaar, "The importance of the Selberg integral", Bull. Amer. Math. Soc. (N. S.), 45:4 (2008), 489-534.

[82] P. J. Forrester, Log-gases and random matrices, London Math. Soc. Monogr. Ser., 34, Princeton Univ. Press, Princeton, NJ, 2010, xiv+791 pp.

[83] F. Colomo, A. G. Izergin, V. E. Korepin, V. Tognetti, "Correlators in the Heisenberg XXO chain as Fredholm determinants", Phys. Lett. A, 169:4 (1992), 243-247.

[84] F. Colomo, A. G. Izergin, V.E. Korepin, V. Tognetti, "Temperature correlation functions in the XX0 Heisenberg chain. I", TMФ, 94:1 (1993), 19-51; Theoret. and Math. Phys., 94:1 (1993), 11-38.

[85] F. Colomo, A. G. Izergin, V. Tognetti, "Correlation functions in the XXO Heisenberg chain and their relations with spectral shapes", J. Phys. A, 30:2 (1997), 361-370.

[86] B.-Q. Jin, V.E. Korepin, "Quantum spin chain, Toeplitz determinants and the Fisher-Hartwig conjecture", J. Statist. Phys., 116:1-4 (2004), 79-95.

[87] D. J. Gross, E. Witten, "Possible third-order phase transition in the large- $N$ lattice gauge theory", Phys. Rev. D, 21:2 (1980), 446-453.

[88] D. Pérez-García, M. Tierz, "Mapping between the Heisenberg XX spin chain and low-energy QCD", Phys. Rev. X, 4:2 (2014), 021050, 12 pp.

[89] P. J. Forrester, S. N. Majumdar, G. Schehr, "Non-intersecting Brownian walkers and Yang-Mills theory on the sphere", Nuclear Phys. B, 844:3 (2011), 500-526.

[90] F. Colomo, A. G. Pronko, "Third-order phase transition in random tilings", Phys. Rev. E, 88 (2013), 042125, 11 pp. 
[91] Г. Бэйтмен, А. Эрдейи, Высшие трансцендентные функиии, т. 1, 2, 3, Наука, M., 1965, 1966, 1967, 294 с., 296 с., 300 с.; пер. с англ.: A. Erdélyi, W. Magnus, F. Oberhettinger, F. G. Tricomi, Higher transcendental functions, v. 1, 2, 3, Based, in part, on notes left by H. Bateman, McGraw-Hill Book Company, Inc., New York-Toronto-London, 1953, 1953, 1955, xxvi+302 pp., xvii+396 pp., xvii+292 pp.

[92] W. Li, H. Park, M. Widom, "Finite-size scaling amplitudes in a random tiling model", J. Phys. A, 23:11 (1990), L573-L580.

[93] W. Li, H. Park, "Logarithmic singularity in the surface free energy near commensurate-incommensurate transitions", J. Phys. A, 24:1 (1991), 257-264.

[94] У. Фултон, Таблицы Юнга и их приложения $к$ теории представлений и геометрии, МЦНМО, М., 2006, 328 с.; пер. с англ.: W. Fulton, Young tableaux. With applications to representation theory and geometry, London Math. Soc. Stud. Texts, 35, Cambridge Univ. Press, Cambridge, 1997, x+260 pp.

[95] J. Harnad, E. Lee, Symmetric polynomials, generalized Jacobi-Trudi identities and $\tau$-functions, CRM-3326, 2013, 33 (v5 - 2013) pp., arXiv: 1304.0020.

[96] Ф.Р. Гантмахер, Теория матрии, 4-е изд., Наука, М., 1988, 549 с.; англ. пер. 1-го изд.: F. R. Gantmacher, The theory of matrices, v. 1, 2, Chelsea Publishing Co., New York, 1959, x+374 pp., ix+276 pp.

[97] Н. М. Боголюбов, К. Малышев, "Корреляционные функции $X X Z$ цепочки Гейзенберга для нулевой или бесконечной анизотропии и случайные блуждания недружественных пешеходов", Алгебра и анализ, 22:3 (2010), 32-59; англ. пер.: N. M. Bogoliubov, C. Malyshev, "The correlation functions of the $X X Z$ Heisenberg chain in the case of zero or infinite anisotropy, and random walks of vicious walkers", St. Petersburg Math. J., 22:3 (2011), 359-377.

[98] Н. М. Боголюбов, К. Л. Малышев, "Изинговский предел $X X Z$-магнетика Гейзенберга и некоторые температурные корреляционные функции”, ТМФ, 169:2 (2011), 179-193; англ. пер.: N. M. Bogoliubov, C. L. Malyshev, "Ising limit of a Heisenberg XXZ magnet and some temperature correlation functions", Theoret. and Math. Phys., 169:2 (2011), 1517-1529.

[99] V. Korepin, J. Terilla, "Thermodynamic interpretation of the quantum error correcting criterion", Quantum Inf. Process., 1:4 (2002), 225-242.

[100] F. C. Alcaraz, R. Z. Bariev, "An exactly solvable constrained XXZ chain", Statistical physics on the eve of the 21 st century, In honour of J. B. McGuire on the occasion of his 65th birthday, Ser. Adv. Statist. Mech., 14, World Sci. Publ., River Edge, NJ, 1999, 412-424.

[101] Н. И. Абаренкова, А. Г. Пронько, “Температурный коррелятор в абсолютно анизотропном $X X Z$-магнетике Гейзенберга", TMФ, 131:2 (2002), 288-303; англ. пер.: N.I. Abarenkova, A. G. Pronko, "Temperature correlation function in the absolutely anisotropic XXZ Heisenberg magnet", Theoret. and Math. Phys., 131:2 (2002), 690-703.

[102] В. О. Тарасов, Л. А. Тахтаджян, Л. Д. Фаддеев, “Локальные гамильтонианы для интегрируемых квантовых моделей на решетке”, ТМФ, 57:2 (1983), 163-181; англ. пер.: V. O. Tarasov, L. A. Takhtadzhyan, L. D. Faddeev, "Local Hamiltonians for integrable quantum models on a lattice", Theoret. and Math. Phys., 57:2 (1983), 1059-1073.

[103] А. Г. Изергин, Н. А. Китанин, Н. А. Славнов, "О корреляционных функциях XY модели", Вопросы квантовой теории поля и статистической физики. 13, Зап. науч. сем. ПОМИ, 224, ПОМИ, СПб., 1995, 178-191; англ. пер.: А. G. Izergin, N. A. Kitanin, N. A. Slavnov, "On correlation functions of the XY-model", J. Math. Sci. (N. Y.), 88:2 (1998), 224-232. 
[104] M. Katori, H. Tanemura, "Scaling limit of vicious walks and two-matrix model", Phys. Rev. E, 66:1 (2002), 011105, 12 pp.

[105] M. Katori, H. Tanemura, T. Nagao, N. Komatsuda, "Vicious walks with a wall, noncolliding meanders, and chiral and Bogoliubov-de Gennes random matrices", Phys. Rev. E, 68:2 (2003), 021112, 16 pp.

[106] T. C. Dorlas, A. M. Povolotsky, V. B. Priezzhev, "From vicious walkers to TASEP", J. Stat. Phys., 135:3 (2009), 483-517.

[107] М.Л. Мета, Случайные матрицы, МЦНМО, М., 2012, 648 с.; пер. с англ.: M. L. Mehta, Random matrices, 2nd ed., Academic Press, Inc., Boston, MA, 1991, xviii+562 pp.

[108] I. Gessel, G. Viennot, "Binomial determinants, paths, and hook length formulae", Adv. in Math., 58:3 (1985), 300-321.

[109] N. M. Bogoliubov, C. Malyshev, "A combinatorial interpretation of the scalar products of state vectors of integrable models", Теория представлений, динамические системы, комбинаторные методы. ХХІІІ, Зап. науч. сем. ПОМИ, 421, ПОМИ, СПб., 2014, 33-46; J. Math. Sci. (N. Y.), 200:6 (2014), 662-670.

[110] E. W. Barnes, "The theory of the G-function", Quart. J. Pure and Appl. Math., 31 (1900), 264-314.

[111] V.S. Adamchik, Contributions to the theory of the Barnes function, 2003, 18 pp., http://repository.cmu.edu/compsci/87.

[112] G. Algara-Siller, O. Lehtinen, F. C. Wang, R. R. Nair, U. Kaiser, H. A. Wu, A. K. Geim, I. V. Grigorieva, "Square ice in graphene nanocapillaries", Nature, 519:7544 (2015), 443-445.

[113] A. Klimyk, K. Schmüdgen, Quantum groups and their representations, Texts Monogr. Phys., Springer-Verlag, Berlin, 1997, xx+552 pp.

[114] J. Goldman, G.-C. Rota, "On the foundations of combinatorial theory. IV. Finite vector spaces and Eulerian generating functions", Studies in Appl. Math., 49:3 (1970), 239-258.

[115] Д. П. Желобенко, А. И. Штерн, Представления групп Ли, Наука, М., 1983, 360 с.

[116] P.P. Kulish, "Quantum difference nonlinear Schrödinger equation", Lett. Math. Phys., 5:3 (1981), 191-197.

[117] R. K. Bullough, N. M. Bogoliubov, G. D. Pang, J. Timmonen, "Quantum repulsive nonlinear Schrödinger models and their 'superconductivity'", Chaos Solitons Fractals, 5:12 (1995), 2639-2656.

[118] C. Korff, "Noncommutative Schur polynomials and the crystal limit of the $U_{q} \widehat{\mathfrak{s l}}(2)$ vertex model", J. Phys. A, 43:43 (2010), 434021, 20 pp.

[119] N. M. Bogoliubov, R. K. Bullough, G. D. Pang, "Exact solution of a $q$-boson hopping model", Phys. Rev. B, 47:17 (1993), 11495-11498.

[120] N. M. Bogoliubov, R.K. Bullough, J. Timonen, "Critical behavior for correlated strongly coupled boson systems in 1+1 dimensions", Phys. Rev. Lett., 72:25 (1994), 3933-3936.

[121] N. Bogoliubov, J. Timonen, "Correlation functions for a strongly coupled boson system and plane partitions", Philos. Trans. R. Soc. Lond. Ser. A Math. Phys. Eng. Sci., 369:1939 (2011), 1319-1333.

[122] В. Г. Дринфельд, "Квантовые группы”, Дифференциальная геометрия, группь Ли и механика. VIII, Зап. науч. сем. ЛОМИ, 155, Изд-во "Наука", Ленингр. отд., Л., 1986, 18-49; англ. пер.: V. G. Drinfel'd, "Quantum groups", J. Soviet Math., 
41:2 (1988), 898-915; Proceedings of the International Congress of Mathematicians, v. I (Berkeley, CA, 1986), Amer. Math. Soc., Providence, RI, 1987, 798-820.

[123] P.P. Kulish, E. V. Damaskinsky, "On the $q$-oscillator and the quantum algebra $\mathrm{su}_{q}(1,1)$ ", J. Phys. A, 23:9 (1990), L415-L420.

[124] N. M. Bogoliubov, A. G. Izergin, N. A. Kitanine, "Correlation functions for a strongly correlated boson system", Nuclear Phys. B, 516:3 (1998), 501-528.

[125] P. Carruthers, M. M. Nieto, "Phase and angle variables in quantum mechanics", Rev. Modern Phys., 40:2 (1968), 411-440.

\section{Николай Михайлович Боголюбов}

(Nikolai M. Bogolyubov)

Санкт-Петербургское отделение

Математического института им. В. А. Стеклова

Российской академии наук;

Санкт-Петербургский национальный

исследовательский университет информационных

технологий, механики и оптики

E-mail: bogoliub@pdmi.ras.ru

\section{Кирилл Леонидович Малышев}

(Cyril L. Malyshev)

Санкт-Петербургское отделение

Математического института им. В. А. Стеклова

Российской академии наук

E-mail: malyshev@pdmi.ras.ru
Поступила в редакцию 31.01 .2015 